PREPARED FOR SUBMission to JHEP

\title{
Effective Field Theories of Post-Newtonian Gravity: A comprehensive review
}

\author{
Michèle Levi ${ }^{a, b}$ \\ ${ }^{a}$ Institut de Physique Théorique, CEA $\&$ CNRS, Université Paris-Saclay, \\ 91191 Gif-sur-Yvette, France \\ ${ }^{b}$ Niels Bohr International Academy, Niels Bohr Institute, University of Copenhagen, \\ Blegdamsvej 17, 2100 Copenhagen, Denmark \\ E-mail: michelelevi@nbi.ku.dk
}


ABstract: This review article presents the progress made over the last decade, since the introduction of effective field theories (EFTs) into post-Newtonian (PN) gravity. These have been put forward in the context of gravitational waves (GWs) from the compact binary inspiral. The mature development of this interdisciplinary field has resulted in significant advances of wide interest to physics at several levels serving various purposes. The field has firmly demonstrated, that seemingly disparate physical domains, such as quantum field theory and classical gravity, are related, and that the EFT framework is a universal one, where it has been proven to supply a robust methodology to boost progress in the development of PN theory. In this review emphasis was put on an accessible pedagogic presentation of the field theoretic aspects of the subject, with the view, that these are in fact common across the whole of theoretical physics, rather than in their original narrow quantum context. The review is aimed at a broad audience, from general readers new to the field, to specialists and experts in related subjects.

The review begins with an overview of the introduction of EFTs into classical gravity and their development. Then, the basic ideas, which form the conceptual foundation of EFTs, are provided, and the strategy of a multi-stage EFT framework, which is utilized for the PN binary inspiral problem, is outlined. The main body of the review is then dedicated to presenting in detail the study of each of the effective theories at each of the intermediate scales in the problem, up to the actual GW observables. First, the EFT for a single compact object is considered, from which one proceeds to the EFT of a compact binary system, viewed as a composite particle with internal binding interactions. Finally, one arrives at the effective theory of the time-dependent multipole moments of the radiating system. The review is concluded with the multiple prospects of building on the progress in the field, and using further modern field theory insights and tools, to specifically address the study of GWs, as well as to broadly expand our fundamental understanding of gauge and gravity theories across the classical and quantum regimes.

KEYwords: Effective Field Theories, Theories of Gravity, Black Holes, gravitational waves 


\section{Contents}

1 EFTs in classical gravity 1

2 Tower of EFTs $\quad 8$

$\begin{array}{lll}2.1 & \text { EFTs for pedestrians } & 10\end{array}$

$\begin{array}{lll}2.2 & \text { Tower of EFTs for GWs } & 12\end{array}$

$\begin{array}{lll}2.2 .1 & \text { One-particle EFT } & 13\end{array}$

$\begin{array}{lll}2.2 .2 & \text { EFT of a composite particle } & 13\end{array}$

$\begin{array}{lll}2.2 .3 & \text { Effective theory of dynamical multipoles } & 15\end{array}$

3 One-particle EFT $\quad 15$

$\begin{array}{lll}3.1 & \text { Point mass } & 16\end{array}$

$\begin{array}{ll}3.2 & \text { Spinning particle } \\ & 3.2 .18\end{array}$

$\begin{array}{lll}3.2 .1 & \text { Minimal coupling } & 19\end{array}$

$\begin{array}{lll}3.2 .2 & \text { Non-minimal coupling } & 21\end{array}$

3.3 Dissipative DOFs 23

4 EFT of a composite particle $\quad 25$

$\begin{array}{lll}4.1 & \text { KK metric and gauge fixing } & 27\end{array}$

$\begin{array}{lll}4.2 & \text { Feynman graphs and calculus } & 29\end{array}$

$\begin{array}{lll}4.3 & \text { Standard action, EOMs, and potentials } & 40\end{array}$

$5 \quad$ Effective theory of dynamical multipoles $\quad 42$

5.1 Radiation, radiation reaction, and RG flows 42

5.2 EFTofPNG public code $\quad 54$

$6 \quad$ Field theory for gravity at all scales $\quad 55$

\section{EFTs in classical gravity}

Gravitational radiation is a probing prediction of any candidate complete theory of gravity. As a relativistic field theory of gravity, the general theory of relativity (GR), which was formulated in 1915 by Einstein, indeed led him soon after to predict gravitational waves (GWs) within GR in 1916. However, the first evidence of GWs arrived only with the discovery of the first binary pulsar by Hulse and Taylor in 1974 [1], where in the analysis, done in the following years, the decay of the orbital period fit with the loss of energy and angular momentum due to gravitational radiation, as expected from GR [2-4]. In the following decades laser interferometers were developed for the purpose of observing GWs directly and making GW astronomy a reality, which led to the construction of a few groundbased GW detectors, all at a frequency range of about $10-10^{4} \mathrm{~Hz}$, appropriate for the 
detection of comparable mass compact binary coalescence (CBC) events: TAMA in Japan, the twin LIGO detectors in the USA [5], and GEO600 [6] and Virgo [7] in Europe. TAMA started operating in 1999, with both LIGO and GEO600 starting in 2002. Later, TAMA was closed and Virgo was put online in 2007. For a decade none of these detectors has achieved detection, and subsequently, LIGO and Virgo were shut down to undergo major upgrading to second-generation GW detectors in order to make them more sensitive.

The first observation of GWs, labeled 'GW150914' [8], was indeed realized in 2015 with the Advanced LIGO detectors, only 4 days before the operation of LIGO's advanced version officially commenced. Three further detections - GW151226 [9], GW170104 [10], and GW170608 [11] - by Advanced LIGO followed, before the Advanced Virgo detector joined the observations in 2017. The first joint detection by these three advanced detectors in the USA and Europe, GW170814 [12], arrived shortly after, allowing for the localization of the source and testing of GW polarizations for the first time. Moreover, a second joint detection, GW170817 [13], was attained just after, this time involving a neutron star (NS) binary merger, after all five previous GW events featured only black hole (BH) binaries. Thus, this was the first multi-messenger detection of a NS merger, with electromagnetic counterparts, in particular the gamma ray burst (GRB) 170817A, which confirmed the longstanding conjecture, that short GRBs originate from NS mergers [14, 15].

These first earthshaking GW detections proved to be even more telling than expected (see, e.g. also $[16,17]$ ), and paved the way for further plans of advanced GW detectors to multiply worldwide. In particular, second-generation ground-based GW detectors, such as KAGRA, the successor of TAMA in Japan [18], and IndiGO in India [19], are expected to start operation in the coming years [20], complementing the distribution of the worldwide network $^{1}$. Furthermore, there are already specific plans for third-generation ground-based detectors, such as the Einstein Telescope in Europe [21], and the Cosmic Explorer in the USA [22]. Additionally, the space-based GW detector LISA [23] is designed to operate in a complementary frequency range of about $10^{-5}-1 \mathrm{~Hz}$, appropriate for the detection of extreme mass ratio inspirals (EMRIs). Moreover, further GW detectors, such as the ground-based SOGRO [24], and the space-based Japanese DECIGO [25] and Chinese TianQin [26], in complementary intermediate frequency ranges, have also been proposed.

The GW detections made so far have all involved the CBC signal, where binary constituents of comparable mass are concerned. The evolution of such a compact binary is comprised of three consecutive yet distinguishable phases [27]: 1 . The inspiral, where the components of the binary are still moving at non-relativistic (NR) velocities, and their orbital separation is slowly decaying; 2. The merger, when the separation between the components falls roughly below the innermost stable orbit of a $\mathrm{BH}$, and the objects reach relativistic velocities, and merge into a single compact object; 3 . The ringdown phase for $\mathrm{BH}$ binaries, where spacetime settles down to that of a rotating Kerr $\mathrm{BH}$ via quasinormal mode oscillations $[28,29]^{2}$. Each of these phases has been properly studied with a

\footnotetext{
${ }^{1}$ We note however, that it would be desirable to further extend the distribution of the worldwide network to the Southern Hemisphere in the future.

${ }^{2}$ For a binary of NSs, there is likely an intermediate stage of a hypermassive metastable NS as the merger product.
} 
distinct methodology, as the physical conditions around the peak amplitude of the CBC event rapidly switch. The initial inspiral phase, where the NR approximation holds, is ideally treated with post-Newtonian (PN) theory [30,31], whereas in entering the merger phase and the strong field regime, PN theory, in principle, breaks down. The merger and ringdown phases are currently studied in detail via numerical simulations, which solve for the fully exact relativistic evolution of the binary [32]. Finally, the ringdown phase can also be analytically treated with $\mathrm{BH}$ perturbation theory and self-force formalism, where the $\mathrm{BH}$ spacetime is considered as slightly deformed by a mass much smaller than the mass of the remnant compact object.

It is important to stress, that while numerical simulations currently exclusively treat the strong field regime in full detail, covering the merger and ringdown phases of the CBC signal, they are inherently not suitable for tackling the inspiral phase. The long inspiral phase makes up the major portion of the CBC signal if observed in its entirety, and depending on the masses in the binary, it can be the only observed signal falling within the frequency band of the detectors. The inspiral phase is also uniquely telling, providing exclusive input on gravity theories in the PN regime, and on the individual components of the binary, yet it can not be treated numerically due to the intrinsic long timescale, which characterizes the evolution at this stage. The regimes of validity of the different and complementary elementary physical methodologies used to study the $\mathrm{CBC}$ event, and their overlap, are illustrated in figure 1, depending on the mass ratio of the components of the binary, and the compactness of the system [33]. The latter is evaluated by the ratio of the total mass of the system to its typical size, i.e. $M / r$, where $M=m_{1}+m_{2}$ is the total mass of the binary, and $r$ is its orbital separation.

Remarkably, even prior to the breakthrough in numerical relativity, which enabled the first exact treatment of the $\mathrm{CBC}$ event in the strong field regime [32], an inclusive theoretical framework, which tackles and models the complete evolution of the compact binary, has already been devised by Buonanno and Damour [34-36]. This framework, dubbed the 'effective one-body' (EOB) approach, provided the first complete theoretical waveform templates for the CBC signal. The idea behind the EOB approach was to relate the first and last phases of evolution, where analytical control has been feasible, via a mapping of the two-body problem to an effective one-body problem, in the spirit of Newtonian physics. This mapping, which thus enables fully tracking the evolution of the system, as it continuously transforms from having two compact objects to a single one, is non-trivially constructed in curved spacetime [36]. Therefore, the EOB framework currently enables extraction of the desirable elements from all the abovementioned elementary methodologies, and to integrate them into the full theoretical CBC template models, that are currently used in the GW detectors. There are additional phenomenological models, which similarly to EOB ones, also combine analytical and numerical results into complete inspiral-mergerringdown waveform models $[37,38]$. An elaborate discussion of the various methodologies used to study and model the CBC signal, and their multiple interfaces, can be found in [33].

As the CBC signal is weak with respect to the sensitivities and various noise sources of current ground-based GW detectors, matched filtering is utilized for the detection of GW 


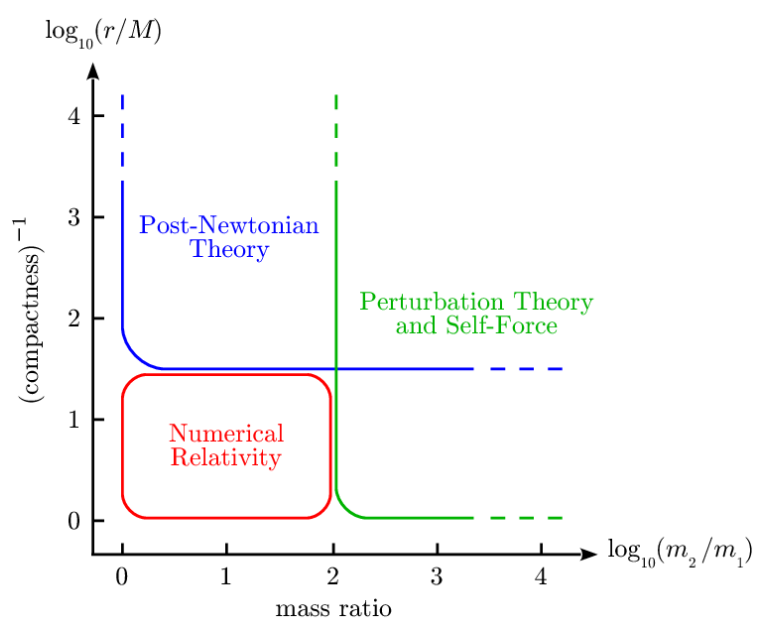

Figure 1. The various elementary analytical and numerical methodologies used to study CBCs, depending on the mass ratio, $0<m_{1} / m_{2} \leq 1$, of the components of the binary, and the compactness of the system, evaluated by the parameter $M / r$, where $M=m_{1}+m_{2}$ is the total mass, and $r$ the typical size of the system. However, in order to model the complete CBC signal, one needs to resort to the comprehensive EOB framework, which enables their integration into the full theoretical GW templates, currently used in the GW detectors. Note that BH perturbation theory also accounts for the case of EMRIs, which will be targeted by the future space-based GW detector LISA. Reproduced with permission from Le Tiec [33].

events, which places high demands on the accuracy of theoretical waveform templates. Moreover, with the expected increasing influx of even more detailed and telling GW data, we have truly entered a new era of high precision gravity. To that end, the framework of effective field theories (EFTs) is as a powerful means to advance theoretical progress, as put forward by Goldberger et al. [39-41]. In particular, EFTs, which are designed for high precision computation, can be applied in the inspiral and ringdown phases of the CBC, where multiple widely separated physical scales are involved, and the evolution is amenable to analytical perturbative treatment. However, the concept of EFTs is inextricably tied to that of renormalization, historically devised in the realm of quantum field theory (QFT) and particle physics, where these and classical gravity are considered as disparate branches of physics. Despite this, the novel EFT approach for gravitational radiation presented in [39-41] soon inspired the use of the EFT framework in some modern problems in classical gravity, where a clear hierarchy of scales also exists. Notably, EFTs were applied to higherdimensional gravity with compact dimensions, inspired by string theory [42-46], or to weak ultra-relativistic scattering [47].

Following the introduction of the EFT formulation for the compact binary inspiral, where the small parameter, controlling the hierarchy of scales, is the NR orbital velocity, $v \ll 1^{3}$, consistent progress was made along this research program in PN theory. After

\footnotetext{
${ }^{3}$ Throughout this review the speed of light, $c$, and the reduced Planck constant, $\hbar$, are taken as $c=\hbar=1$, such that the Planck mass, $m_{p}$, and the gravitational constant, $G$, are normalized via $m_{p}^{2} \equiv \frac{1}{32 \pi G}$.
} 
the first PN $(1 \mathrm{PN})$ order $^{4}$ correction in the conservative orbital dynamics was reproduced ${ }^{5}$ $[39,41]$, and was simplified with a useful Kaluza-Klein (KK) reduction of the spacetime metric [48-50], higher PN order corrections in the point-mass sector were reproduced: The $2 \mathrm{PN}$ order [51, 52], where in [52] it was first considered within a generic $n$-body problem, and an automatization of the computations was put forward, as well as the 3PN order [53]. Subsequently, the current state of the art at the $4 \mathrm{PN}$ order was approached in [54], and in parallel with the progress and completion of this sector, attained via other PN methodologies [30], it was partly computed in [55], making use of the analogy with amplitudes of massless gauge theory. Moreover, after the introduction of the effective worldline massinduced action of a single compact object in [39-41], its non-minimal coupling part has been extended in [56]. Furthermore, the Wilson coefficients of this part of the effective action were studied for BHs in an arbitrary dimension in [57], where a classical renormalization group ( $\mathrm{RG}$ ) flow was found at higher dimensions.

However, the knowledge of spin effects in the compact binary inspiral is also of crucial importance to gravity, quantum chromodynamics (QCD), and astrophysics (see, e.g. [58, 59]). Spin is one of the two unique features of BHs in nature, along with the mass, whereas spin effects in NSs are required in order to learn about their internal structure [60], which in turn is also expected to reveal information on QCD and strong gravity. Indeed, spin effects in the orbital dynamics were first tackled within the EFT approach in [61], where leading order $(\mathrm{LO})$ corrections at the $1.5 \mathrm{PN}$ and $2 \mathrm{PN}$ orders ${ }^{6}$ were reproduced. Subsequently, next-to-leading order (NLO) corrections at the 3PN order were approached in [62-65], and reproduced in [66-68] at the 2.5PN order, where in [65] finite size effects were tackled, and in $[64,68]$ the explicit relations to corresponding Hamiltonians were provided. Moreover, next-to-next-to-leading order (NNLO) corrections [69-72] as well as LO ones [73] at the 4PN order were obtained. Further, NNLO corrections [74] and LO ones [73] at the 3.5PN order were reproduced, where in [71-73] finite size effects were considered. This state-of-the-art PN accuracy in the spin sector was based on the EFT formulation for spinning objects presented in [75, 76], building on [39, 41, 64, 68, 70], where in [75] all spin effects up to NLO were consistently reproduced. It should be noted, that a distinct EFT formulation for general gravitating rotating objects was provided in [77], which is formally and phenomenologically complementary to that in [75], and was not specified in the PN context.

Dissipative effects of the components of the inspiraling compact binary, such as the absorption of gravitational energy by the horizon of BHs, or the dissipative tidal deformations of NSs, also have an effect on GW emission. For astrophysical objects both finite size and

\footnotetext{
${ }^{4}$ The $n \mathrm{PN}$ order is the $(v / c)^{2 n}$ order correction in GR to Newtonian gravity.

${ }^{5} \mathrm{~A}$ comprehensive account of PN theory methodologies and results can be found in the seminal review by Blanchet [30], as well as the recent review by Schäfer and Jaranowski [31], whose focus was solely on Hamiltonian formulations and results. Though the progress made in the EFT field presented here belongs to the advances in PN theory, the latter constitutes a monumental body of work, which is beyond the limited scope of this review.

${ }^{6}$ The explicit PN order of spin effects is evaluated, in accordance with the standard practice found in the templates for the LIGO/Virgo detectors, for rapidly rotating compact objects, particularly for near extremal Kerr BHs.
} 
dissipative effects provide an indirect probe of the microphysics of their internal structure. Thus, the absorption by BH horizons was considered and reproduced in the EFT framework in [78-80], where the leading PN dissipative effect is superradiance of rotating BHs at the 5PN order. More general dissipative effects of arbitrary rotating gravitating objects were considered in [81, 82], based on the EFT formulation in [77], which are expected to contribute at higher PN orders. Tidal deformations of non-rotating gravitating objects were also treated in [56] via an effective action approach. Finally, dynamical tidal deformations of non-rotating objects, in particular resonances in NSs, were treated in [83-85].

The direct effects of the gravitational radiation, emitted from the binary system, were first formulated and treated in detail in the EFT framework in [86], within an effective theory with a set of multipole moments of the system, which sources the radiation. Next, general expressions for the multipole moments, radiated power, and GW amplitude, were derived from a general multipole expansion of the action in [87]. The 1PN order correction to an infinite subset of the multipoles was derived explicitly in [88, 89], and NLO multipole moments, that depend on the spins of the compact components of the binary, were tackled in $[90,91]$. Hereditary tail effects, where the radiation emitted from the binary is scattered by its self-generated gravitational background, were studied and reproduced in [86], where a classical RG flow of the mass quadrupole of the binary was found. A subleading RG flow of the total mass of the system was uncovered in [92].

Subsequently, radiation reaction, which is the backreaction of the gravitational radiation, emitted from the system, on the system itself, should also be taken into account. In fact, the treatment of $\mathrm{PN}$ radiation reaction in compact binary inspirals is in close analogy with the self-force in EMRIs, where there is a small mass, $m$, moving in the gravitational background of a large mass, $M$ (see, e.g. a recent review in [93]). EMRIs are studied perturbatively in terms of the small mass ratio, $m / M \ll 1$, where one considers the self-forces acting on the small mass due to its own effect on the gravitational background, generated by the large mass. Moreover, examining the test particle limit is similar to considering EMRIs with $\mathrm{BH}$ perturbation theory and self-force, and it provides information on the ringdown phase of the comparable mass $\mathrm{CBC}$ in the strong field regime. Self-force in EMRIs was first studied in an EFT approach in [94], where the LO correction was reproduced ${ }^{7}$. The self-force in EMRIs was further studied within the EFT framework at higher orders in the mass ratio in a non-linear scalar theory model in [95, 96]. Moreover, the self-forces in the ultra-relativistic limit, and in non-vacuum spacetimes, were investigated within an EFT approach in [97], and [98], respectively.

The radiation reaction in the compact binary inspiral with components of comparable mass was then tackled in an EFT approach in [99], where the LO effect at the 2.5PN order was reproduced. Next, the NLO correction at the 3.5PN order, an effect still linear in $G$, was reproduced in [100]. The leading non-linear tail effect, which yields a radiation reaction effect at the $4 \mathrm{PN}$ order, was first approached in [101], and further studied in [102], using a formulation for classical causal actions of generic dissipative systems, which

\footnotetext{
${ }^{7}$ However, the primary obstacle here is that the relevant Green's functions in the BH spacetime background are highly non-trivial, and require numerical computation, so one would have to find out how to implement a semi-analytic EFT.
} 
was put forward in [103, 104]. An independent formulation of classical causal actions for the study of radiation and the radiation reaction, which can also be generally applied at the level of the equations of motion (EOMs), was devised in [88, 89], and extended for a general spacetime dimension in $[105,106]$. The leading non-linear radiation reaction effect contributes logarithmic corrections to the binding energy, which were first derived via the EFT approach in [92], though not through an analysis of the radiation reaction. Lastly, the leading radiation reaction correction linear in the spin of the objects at the $4 \mathrm{PN}$ order was reproduced in [107], while the related LO spin-orbit tail effect was previously reproduced in [91]. The leading radiation reaction correction quadratic in the spins at the 4.5PN order, including spin-squared finite size effects, was derived in [108].

All in all, as outlined above, the mature development of this interdisciplinary field over the last decade, initiated in [39, 41], has resulted in significant advances of wide interest to physics at several levels serving various purposes. First, the field has brought novel perspectives to such important concepts of theoretical physics as renormalization, and the RG. The most fundamental lesson, which this field has validated, is how much the EFT framework is in fact a universal one, apt at describing an unprecedented scope of physical phenomena. As long as a hierarchy of scales, widely separated by a small ratio parameter, is identified in a problem prone to non-linear perturbations, EFTs can be used, whether the non-linear coupling involves the quantum parameter, $\hbar$, or any other nonquantum parameter. The EFT scheme provides a systematic methodology for constructing an effective theory, which describes the physics at some relevant scale to arbitrarily high accuracy. It organizes the perturbative calculation efficiently by employing powerful tools, which are standard in QFT, such as Feynman diagrams and calculus. It also provides a natural framework to handle the regularization required at higher-order perturbative corrections within the standard renormalization scheme. Thus, the universality of the EFT framework is extremely useful, as multi-scale problems are abundant across all of physics. Consequently, the field has instigated the widespread use of the EFT framework in classical gravity in several modern problems, which are in fact motivated by high energy physics, and also address the attempt to arrive at a complete theory of gravity across all scales.

The impact of the field within the definite domain of PN theory and GWs has also been established and varied. Goldberger et al. have put forward an original self-contained EFT program to tackle the intricate PN binary inspiral problem. It should be noted though that some ingredients, inherently included in the EFT methodology, have appeared in some form (which bears some resemblance) in past work [109], or specifically in the domain, e.g. [110, 111]. In the EFT formulation a manifest power counting in the small PN expansion parameter was achieved by performing a decomposition of the gravitational field into modes with definite scaling properties, at the level of the action. Further, the use of action formalism and symmetries, intrinsic in the EFT framework, was invigorated in the various traditional PN methodologies. The tackling of several PN effects via the EFT framework propelled further progress in other PN methodologies in parallel. Notable within the progress made in the field is the improvement in the general understanding of classical spins in gravity, and consequently also several new results in the spinning 
sectors, some of which so far exclusively obtained via the EFT methodology. Some of the remarkable findings within the field include the classical RG flows, and related higher PN order logarithm corrections, which also constitute a unique prediction arising from the EFT framework. The final marked advance in the field is the 'EFTofPNG' public code, which incorporates the EFT framework for high precision computation in PN gravity, including spin effects [112]. Beyond its contribution at the strict level of computational and mathematical physics, it is an accessible tool for the broader community, which also practically fills in the current analytic gap for this significant part of the gravitational waveform models. Notably, this is the first comprehensive code in PN theory to be made public, and hence complements current public codes for GWs, which include numerical codes, codes for BH perturbation theory [113], and codes for data analysis.

However, the field continues to develop. In particular, further progress is desirable in the non-conservative sectors of $\mathrm{PN}$ theory. Building on the progress in the field, the various aspects to be advanced within the context of GWs, in addition to the abundant potential of using further timely field theory advances to improve our understanding of gravity at all scales, are elaborately discussed in section 6 .

This review article is aimed at a broad audience, from general readers new to the field, to specialists and experts in related subjects. In this review emphasis was put on an accessible pedagogic presentation of the field theoretic aspects of the subject, with the view, that these are in fact common across the whole of theoretical physics, rather than in their original narrow quantum context. The review presents the progress made in the field since the introduction of the EFT approach to the PN binary inspiral, where works that served as mileposts are spelled out in more detail. Different reviews in the field of EFTs in PN gravity, highlighting various aspects of it from several perspectives, are found in $[40,41,114-116]$.

The outline of this review is as follows. Section 2 provides the basic ideas, which form the conceptual foundation of EFTs, and outlines the strategy of a multi-stage EFT framework, which is deployed in the PN binary inspiral problem. This section actually serves as the formal complement of the introductory section. The main body of the review is then dedicated to presenting in detail the study of each of the effective theories at each of the intermediate scales in the problem, up to the actual GW observables: From the EFT of the single compact object in section 3, on to that of the binary, viewed as a composite particle with internal binding interactions, in section 4 , to the final effective theory of the time-dependent multipole moments of the radiating system in section 5. The review is concluded in section 6 with the multiple prospects of building on the progress in the field, and using further modern field theory insights and tools, to specifically address the study of GWs, as well as to expand our fundamental understanding of gauge and Gravity theories at all scales.

\section{Tower of EFTs}

Let us first establish the hierarchy of scales in the binary inspiral problem, as depicted in figure $2[39,41]$. Since each isolated object in the binary is compact, with a characteristic 


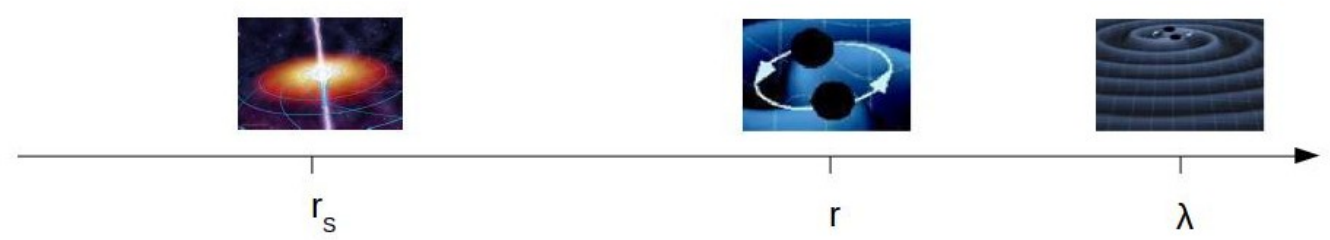

Figure 2. The hierarchy of scales in the binary inspiral problem: $r_{s}$, the scale of the single compact object; $r$, the scale of the orbital separation between the components of the binary; $\lambda$, the wavelength of radiation, emitted from the inspiraling binary; it holds that $r_{s} \lll r \ll \lambda$. To eliminate the smallest scale of the single object, we construct the one-particle EFT. Next, we explicitly integrate out the field modes at the orbital separation scale, to be subsequently matched to the EFT of the composite particle, that is, the binary system. Finally, we eliminate the scale of radiation by explicitly integrating out the radiation field modes.

mass $m$, it holds that its gravitational radius, $G m$, is of the order of the characteristic scale of its internal structure, $r_{s}$, i.e. $r_{s} \sim G m$. For a PN binary system, namely a gravitationally bound one, where the motion of the components has typical NR velocities, $v \ll 1$, the virial theorem holds, such that $G m / r \sim v^{2}$, where $r$ is the typical orbital separation of the bound system. The binary system emits gravitational radiation, e.g. the quadrupole radiation at $\mathrm{LO}$, and so the radiation frequency is fixed by the orbital frequency of the binary, $\omega$. Since the observed radiation consists of on-shell field modes, i.e. such that their momentum, $\left(k_{0}, \vec{k}\right)$, satisfies $k_{0}=|\vec{k}| \equiv k$, it holds that the radiation wavelength, $\lambda$, satisfies $\lambda^{-1} \sim k \sim \omega \sim v / r$. All in all, the three characteristic scales, $r_{s}, r$, and $\lambda$, satisfy the following hierarchy:

$$
r_{s} \sim r v^{2} \sim \lambda v^{3}
$$

such that the mass, $m$, is the only scale in the full theory, where the NR velocity, $v$, is the small parameter, that controls the effective theories at different scales. It should also be noted, that once spin is also taken into account, there is an additional scalar, that is the spin length, $S^{2}$, and yet it holds that $S \lesssim m^{2}$.

To arrive at the orbital dynamics and GW emission observables, we eliminate each of the scales in our problem, one at a time, by constructing an effective theory, where each scale is integrated out, such that we go through three intermediate stages in a tower of effective theories. There are in fact two generic procedures to construct an EFT, referred to as the 'bottom-up' and 'top-down' approaches. They differ in how the effective action, which formally represents the EFT, is obtained. In the bottom-up approach the effective action is constructed from scratch as an infinite series of operators, consisting of the degrees of freedom (DOFs), and constrained by the symmetries, identified at the relevant scale. The top-down approach, on the other hand, arrives at the effective action by explicitly eliminating DOFs from the full action of the small scale (high energy) theory.

In the binary inspiral problem we apply both of these procedures to construct the effective theories in our tower, as detailed in sections 3, 4, and 5. Indeed, in section 2.2 we outline the general strategy, which is deployed in this problem, to ultimately arrive at the orbital dynamics and GW emission observables through three intermediate stages. 
However, before we do that, let us open with a simple QFT prelude in section 2.1, which provides an idea of how a tower of EFTs works and on the fundamental concepts involved [117]. We assume familiarity with the basics of QFT.

\subsection{EFTs for pedestrians}

Let us consider the interacting $\phi^{4}$ theory, given by the following Lagrangian:

$$
\mathcal{L}(\phi)=\frac{1}{2}\left(\partial_{\mu} \phi\right)^{2}+\frac{1}{2} m^{2} \phi^{2}+\frac{\lambda}{4 !} \phi^{4},
$$

with some sharp ultraviolet (UV) momentum cutoff, $\Lambda$, and find the EFT, which corresponds to lowering the cutoff of the theory to $b \Lambda$, where $b$ is some parameter, such that $0<b<1$.

To this end, we proceed to apply Wilson's approach to renormalization to this simple theory, and integrate over the momentum shell. Let us first redefine the field according to its Fourier modes, $\phi(k)$, which will become the integration variable in a functional integral, in the following manner:

$$
\phi(k) \equiv\left\{\begin{array}{ll}
\phi(k) & |k|<b \Lambda \\
0 & |k| \geq b \Lambda
\end{array} \quad, \quad \hat{\phi}(k) \equiv\left\{\begin{array}{ll}
\phi(k) & b \Lambda \leq|k|<\Lambda \\
0 & \text { Otherwise }
\end{array},\right.\right.
$$

where we have also defined $\hat{\phi}$, which represents the high momentum DOFs, that we want to integrate out, and the original field, $\phi$, has been decomposed into two distinct orthogonal momentum components by transforming $\phi \rightarrow \phi+\hat{\phi}$. Let us then rewrite the theory in terms of the two distinct components of field modes:

$$
\begin{aligned}
\mathcal{L}(\phi, \hat{\phi})=\mathcal{L}(\phi)+\frac{1}{2}\left(\partial_{\mu} \hat{\phi}\right)^{2} & +\frac{1}{2} m^{2} \hat{\phi}^{2} \\
& +\lambda\left(\frac{1}{6} \phi^{3} \hat{\phi}+\frac{1}{4} \phi^{2} \hat{\phi}^{2}+\frac{1}{6} \phi \hat{\phi}^{3}+\frac{1}{4 !} \hat{\phi}^{4}\right) .
\end{aligned}
$$

We can now carry out integration over the high momentum DOFs to arrive at an EFT with the cutoff $b \Lambda$, where $\mathcal{L}_{\text {eff }}(\phi)$ is actually defined by a functional integral, as follows:

$$
\int \mathcal{D} \phi \exp \left(-\int d^{d} x \mathcal{L}_{\mathrm{eff}}(\phi)\right) \equiv \int \mathcal{D} \phi \int \mathcal{D} \hat{\phi} \exp \left(-\int d^{d} x \mathcal{L}(\phi, \hat{\phi})\right),
$$

where the functional integral is considered in the Euclidean form, i.e. after Wick rotation.

The integral over the Fourier component $\hat{\phi}$ is performed using the standard QFT perturbative method, involving the extraction of Feynman rules from the action, followed by a diagrammatic expansion of the exponential on the right hand side (RHS) of equation (2.5). Thus we read off the propagator $\langle\hat{\phi} \hat{\phi}\rangle$ from the kinetic term of $\hat{\phi}$ in equation (2.4), and all the following terms in this equation are regarded as perturbations. Note that this also includes the mass term, since the situation considered here is $m^{2} \ll \Lambda^{2}$. Then, the Feynman graphs generated in the expansion are as depicted ${ }^{8}$ in figure 3 . Integrating over

\footnotetext{
${ }^{8}$ All Feynman graphs in this manuscript were created using JaxoDraw [118, 119], based on Axodraw $[120]$.
} 


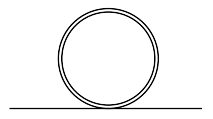

(a)

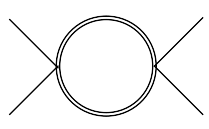

(b)

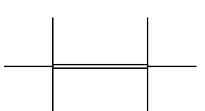

(c)

Figure 3. A $\phi^{4}$ EFT through Wilson's approach: $\hat{\phi}$, the high momentum field component is represented by a double line, while $\phi$, the low momentum component, is represented by a single line. Note that the former will always appear as an internal line, whereas the latter will always appear as external. Diagram (a) is evaluated as $\frac{1}{2} \rho \phi^{2}$, where the new coefficient, $\rho$, gives a correction to the mass parameter, $m^{2}$. Diagram (b) is evaluated as $\frac{1}{4 !} \zeta \phi^{4}$ at LO, where $\zeta$ is a correction to the coupling $\lambda$. Diagram (c) generates a new $\phi^{6}$ interaction with a new coupling constant, $\kappa$; and so on.

$\hat{\phi}$ in such a manner leads to the following theory for $\phi$ with $|k|<b \Lambda$ :

$$
\begin{aligned}
\mathcal{L}_{\text {eff }}(\phi)= & \frac{1}{2}\left(\partial_{\mu} \phi\right)^{2}+\frac{1}{2} m^{2} \phi^{2}+\frac{\lambda}{4 !} \phi^{4} \\
& +(\text { sum of connected diagrams }) \\
= & \frac{1}{2}\left(\partial_{\mu} \phi\right)^{2}+\frac{1}{2}\left(m^{2}+\rho\right) \phi^{2}+\frac{1}{4 !}(\lambda+\zeta) \phi^{4}+\frac{1}{4} \eta \phi^{2}\left(\partial_{\mu} \phi\right)^{2}+\kappa \phi^{6}+\ldots,
\end{aligned}
$$

where the sum is only over connected diagrams, since those are exponentiated to also yield the disconnected ones in the expansion of equation (2.5). The diagrammatic contributions include corrections to the original coupling constants, $\mathrm{m}^{2}$ and $\lambda$, as well as operators of higher mass dimension with new interactions and coupling constants. It should be noted, that there are also derivative interactions from taking into account the external momenta of the diagrams via their Taylor expansion. Indeed, one finds, that the effective Lagrangian, $\mathcal{L}_{\text {eff }}(\phi)$, equals the original Lagrangian, $\mathcal{L}(\phi)$, plus new interaction terms, which compensate for the removal of the high momentum field DOFs.

In general, the procedure of explicitly integrating out the high momentum component, $\hat{\phi}$, generates all possible interactions of the low momentum field modes, $\phi$, and their derivatives, allowed by the symmetries of the effective theory. This means, that one can arrive at the same result, i.e. EFT, just by augmenting the form of the original Lagrangian through writing down an infinite series of all possible operators of higher mass dimension, which satisfy the symmetries, that apply below the new cutoff scale. The original and new operators will be preceded by new coupling constants, which encapsulate the UV information, that has been suppressed from the new effective theory, like the coefficients computed explicitly in figure 3 of the operators $\phi^{2}, \phi^{4}, \phi^{6}$, and so on. In EFT terminology these coupling constants are referred to as Wilson coefficients. What we have just described, in fact, constitutes the content of the decoupling theorem, which states that physics at different scales factorizes, and that the UV physics can be safely suppressed and accounted for in a systematic manner [121].

One can now recognize the two approaches to tackle an EFT, that were alluded to in the introduction of this section: The top-down procedure, which corresponds to Wilson's approach, where one explicitly integrates out high momentum DOFs from a small scale theory, as outlined above, and the bottom-up approach, which relies on the universality 
of the decoupling theorem. The latter can always be deployed, whereas for the top-down approach, the full theory at the small scale should be known, and amenable to perturbative treatment. If possible, the two approaches can then be applied in parallel, as is actually demonstrated in our problem here, in what is described in section 2.2 as the second stage in the tower of EFTs, where we construct an EFT for the binary system as a composite particle. Using the two approaches can, in fact, be one way of fixing the Wilson coefficients, which encode the coupling to the UV scales, a procedure referred to as matching. Otherwise, the matching of the Wilson coefficients can be done by using data from experiments.

Let us further consider the new action in equation (2.6) by rescaling distances and momenta according to $x^{\prime}=x b, k^{\prime}=k / b$, with the new cutoff parameter, $b$. Then the field, $\phi$, can also be rescaled, such that the unperturbed action returns to its initial form, while the various perturbations undergo a transformation, that changes the coupling parameters of the Lagrangian. Hence the combination of integrating out high momentum DOFs with the rescaling of distances and momenta can be rewritten as a transformation of the Lagrangian. One can then further lower the cutoff, and repeat a similar procedure iteratively. Such continuously generated transformations of Lagrangians are referred to as the renormalization group (RG). They do not form a group in the strict mathematical sense, since the operation of integrating out DOFs is not invertible (resolution is lost). However, they renormalize the fields and coupling constants of the theory. There is a universal function, $\beta$, related to the shift in the field strength and coupling constants, that compensates for the shift in the renormalization scale of the theory, $\mu$. The $\beta$ function is just the rate of the $R G$ flow of the coupling constants. For the coupling parameter $\lambda$ in our example here, e.g., the $\beta$ function reads as follows:

$$
\beta(\bar{\lambda})=\mu \frac{d}{d \mu} \bar{\lambda}(\mu)
$$

where $\bar{\lambda}$ is referred to as the running coupling constant, and this is the RG equation .

\subsection{Tower of EFTs for GWs}

Equipped with the basic concepts of renormalization and EFTs, let us now get back to the compact binary inspiral problem. Having identified the problem as a multi-scale one, with three widely separated scales, we now realize that it is ideal to employ the EFT framework to remove each of these scales, one at a time: We can integrate them out in three stages, thereby constructing a tower of EFTs, that eventually leads to the orbital dynamics and GW emission observables. At the first stage we construct the one-particle EFT to remove the small scale of the single isolated compact object. At the next stage we integrate out the orbital scale in order to arrive at the EFT of a composite particle, namely the binary system. Finally, in order to obtain the effects, that involve the radiation directly, we integrate out the radiation scale, which yields an effective theory of dynamical multipole moments. Let us describe here briefly each of these three stages, on which each of the following detailed sections 3,4 , and 5 , respectively, elaborate. 


\subsubsection{One-particle EFT}

As we noted, the first goal is to remove the scale of the single compact object, $r_{s}$, from the purely gravitational action of an isolated compact object (i.e. outside of its interior). For the theory of GR this means starting from the following full theory, given by the Einstein-Hilbert action for the gravitational field, $g_{\mu \nu}(x)$ :

$$
S\left[g_{\mu \nu}\right]=-\frac{1}{16 \pi G} \int d^{4} x \sqrt{g} R\left[g_{\mu \nu}\right]
$$

To this end we take the bottom-up approach in order to attain a one-particle EFT, where we decompose the metric into two distinct momentum components, $g_{\mu \nu} \equiv g_{\mu \nu}^{s}+\bar{g}_{\mu \nu}$, and $g_{\mu \nu}^{s}$ denotes the strong field modes to be removed from the theory. Thus, we construct the one-particle EFT to describe the single object at the orbital scale by introducing an infinite series of worldline operators, which contain new worldline DOFs, that depend on a worldline parameter, $\sigma$. These operators are added to the initial action form in equation (2.8) as additional interactions of the new DOFs with the gravitational field component, $\bar{g}_{\mu \nu}$, such that the new action is of the form:

$$
\begin{aligned}
S_{\mathrm{eff}}\left[\bar{g}_{\mu \nu}(x), y^{\mu}(\sigma), e_{A}^{\mu}(\sigma)\right] & =S\left[\bar{g}_{\mu \nu}\right]+S_{\mathrm{pp}}\left[\bar{g}_{\mu \nu}(y), y^{\mu}(\sigma), e_{A}^{\mu}(\sigma)\right] \\
& =-\frac{1}{16 \pi G} \int d^{4} x \sqrt{\bar{g}} R\left[\bar{g}_{\mu \nu}\right]+\sum_{i=1}^{\infty} C_{i}\left(r_{s}\right) \int d \sigma \mathcal{O}_{i}(\sigma) .
\end{aligned}
$$

In this action we noted the generic new worldline DOFs, $y^{\mu}$, and $e_{A}^{\mu}$, corresponding to the position, and rotation of the object, respectively, and we refer to the new part of the effective action localized on the worldline as the point-particle action, $S_{\mathrm{pp}}$.

In the point-particle action all the UV physics goes into the Wilson coefficients, $C_{i}$, which depend on the only scale in the full theory, $r_{s} \sim m$, and precede the worldline operators $\mathcal{O}_{i}$, which depend only on the scale of the effective theory, i.e. $r$. The terms in the point-particle action in equation (2.9) are thus inserted in increasing orders of the ratio $r_{s} / r$, i.e. according to their $\mathrm{PN}$ relevance. Hence, the crucial task at this stage is to correctly identify the pertinent DOFs, and the symmetries at this scale, both of spacetime and of the object, and to construct all possible worldline operators, which couple the DOFs in all ways allowed by the symmetries. However, we note that the initial theory in equation (2.8) is the full theory for a BH, but for generic compact objects, one should actually add a matter action, i.e. some fluid description of matter. This part of the initial action will also be integrated out, leading to a point-particle action augmented by harmonic oscillator matter DOFs, which are coupled to tidal forces [75]. Finally, the Wilson coefficients are to be matched from the full theory to the one-particle EFT, where the detailed construction of the point-particle action in its explicit form is presented in section 3.

\subsubsection{EFT of a composite particle}

At this subsequent stage we construct the EFT of a composite particle, namely the binary system, and remove the orbital separation scale, $r$. To reach this goal, both the topdown and bottom-up approaches used to construct EFTs, which are mentioned in section 
2.1, are deployed here. We can write the gravitational field, $\bar{g}_{\mu \nu}$, as an expansion on the asymptotic flat spacetime, $\bar{g}_{\mu \nu}=\eta_{\mu \nu}+H_{\mu \nu}+\widetilde{h}_{\mu \nu}$, where $H_{\mu \nu}$ denotes the orbital or 'potential' modes, and $\widetilde{h}_{\mu \nu}$ denotes the radiation modes. The orbital modes are those that mediate the gravitational interaction between the two objects, whereas the radiation modes consist of the on-shell gravitons emitted from the system, and measured by the asymptotic observer, i.e. the GW detectors. Let us note then the scale dependence of these two different components, which is given by

$$
\partial_{t} H_{\mu \nu} \sim \frac{v}{r} H_{\mu \nu}, \quad \partial_{i} H_{\mu \nu} \sim \frac{1}{r} H_{\mu \nu}, \quad \partial_{\mu} \widetilde{h}_{\mu \nu} \sim \frac{v}{r} \widetilde{h}_{\mu \nu}
$$

so the orbital modes are hard momentum modes with respect to the radiation modes.

We start from the full theory at small scales, which is at this stage the one-particle EFT in equation (2.9), that we constructed in the previous stage. As we write the theory here for the two objects, we include a copy of the point-particle action in equation (2.9) for each of the objects in the initial action, namely a two-particle theory:

$$
S_{\mathrm{eff}}\left[\bar{g}_{\mu \nu}, y_{1}^{\mu}, y_{2}^{\mu}, e_{1 A}^{\mu}, e_{2 A}^{\mu}\right]=S\left[\bar{g}_{\mu \nu}\right]+\sum_{a=1}^{2} S_{p p}\left[\bar{g}_{\mu \nu}\left(y_{a}\right),\left(y_{a}\right)^{\mu},\left(e_{a}\right)_{A}^{\mu}\right]\left(\sigma_{a}\right) .
$$

We integrate out explicitly the orbital modes from the full two-particle theory in equation (2.11) by using standard perturbative methods in QFT, with a diagrammatic expansion. Then, the effective action of the composite particle is defined, similar to equation (2.5), by the following functional integral:

$$
e^{i S_{\mathrm{eff}}\left[\widetilde{g}_{\mu \nu},\left(y_{c}\right)^{\mu},\left(e_{c}\right)_{A}^{\mu}\right]} \equiv \int \mathcal{D} H_{\mu \nu} e^{i S_{\mathrm{eff}}\left[\bar{g}_{\mu \nu}, y_{1}^{\mu}, y_{2}^{\mu}, e_{1 A}^{\mu}, e_{2 A}^{\mu}\right]}
$$

where $\widetilde{g}_{\mu \nu} \equiv \eta_{\mu \nu}+\widetilde{h}_{\mu \nu}$, and the subscript 'c' denotes the generic worldline DOFs of the composite object, similar to the worldline DOFs of a single object in equation (2.9). The functional integral is considered here only in the classical limit, where the relevant Feynman diagrams to evaluate in the expansion are graphs, which are tree level in the fields ${ }^{9}$.

At the same time a bottom-up approach is taken, and the effective action of a single composite particle, coupled to the gravitational radiation field, $\widetilde{g}_{\mu \nu}$, is written as follows $[86,87]:$

$$
S_{\mathrm{eff}}\left[\widetilde{g}_{\mu \nu},\left(y_{c}\right)^{\mu},\left(e_{c}\right)_{A}^{\mu}\right]=-\frac{1}{16 \pi G} \int d^{4} x \sqrt{\widetilde{g}} R\left[\widetilde{g}_{\mu \nu}\right]+S_{\mathrm{pp}(\mathrm{comp})}\left[\widetilde{g}_{\mu \nu},\left(y_{c}\right)^{\mu},\left(e_{c}\right)_{A}^{\mu}\right]\left(\sigma_{c}\right),
$$

where $S_{\mathrm{pp}(\mathrm{comp})}$ is the effective worldline action at the radiation scale, which describes the composite object with a suitable 'center of object' coordinate, $\left(y_{c}\right)^{\mu}$, and a tetrad $\left(e_{c}\right)_{A}^{\mu}$. The explicit form of the effective worldline action, $S_{\mathrm{pp}(\mathrm{comp})}$, is given in section 5.1, similar

\footnotetext{
${ }^{9} \mathrm{~A}$ graph with a graviton loop is suppressed by a factor of the ratio $\hbar / L \sim\left(m_{p} / m_{\odot}\right)^{2} \approx 10^{-76}$, where $L$ is the angular momentum of a compact object, and $m_{\odot}$ is the solar mass. Hence graviton loops can be safely neglected, see also figure 4 . However, it should be stressed that the diagrammatic expansion involves loop integrals due to the non-linearity of GR in the gravitational constant, $G$, i.e. the self-interaction of the field in classical gravity.
} 
to the generic structure in equation (2.9), where the multipole moments of the composite object are regarded as the Wilson coefficients of the theory.

The matching of the Wilson coefficients of the EFT of the composite particle in equation (2.13) is done by explicit perturbative computation from the full two-particle theory as in equation (2.12). This perturbative computation consists of two types of Feynman diagrams: Graphs, which contain only internal orbital field modes between the two objects, with no external radiation field modes, and account for the conservative sector of the theory, from which the orbital dynamics is derived, and which is presented in detail in section 4 ; and graphs, which contain a single external radiation field mode, that are matched onto the radiative sector of the theory, and are discussed in the beginning of section 5.1.

\subsubsection{Effective theory of dynamical multipoles}

At this final stage we are concerned with effects, involving the radiation directly. In the conservative sector, where no radiation modes, $\widetilde{h}$, are present, the EFT progression is actually done after the two previous stages: One just needs to further process the resulting set of interaction potentials, which enable derivation of the EOMs for the constituents of the binary, the Hamiltonian potentials, the binding energy, and other gauge invariant observables, as discussed in sections 4.3 and 5.2. Otherwise, where radiation modes are involved, explicit perturbative computations are carried out, essentially taking the topdown approach. Here one starts with the full theory from the previous stage in equation (2.13), of a composite particle with a set of multipole moments at the radiation scale, $\lambda$.

These computations involve Feynman graphs with a single worldline of the composite particle, where radiation modes are integrated out, such that eventually there are no field DOFs left in the theory. However, since at this stage the system is dissipative, and time reversal no longer holds, the action in equation (2.13) should in fact be adjusted in order to describe the non-conservative evolution. This is indeed attained via the closed time path (CTP) formalism, and the corresponding use of retarded propagators, which is presented in detail in section 5.1. With this developed formulation of the action, one can compute the GW energy flux, gravitational waveform, tail effects, radiation reaction forces, and RG flows of the time-dependent multipole moments. Thus, after all radiation modes are integrated out, one is left with an effective theory of the set of dynamical multipole moments of the binary system. This eventual stage of the tower is discussed in detail in section 5 .

\section{One-particle EFT}

In this section we discuss in detail the construction of the one-particle EFT presented in equation (2.9). This EFT removes the short scale of the internal structure of the isolated compact object, $r_{s}$, and provides a description to a single object coupled to gravity, that holds at the orbital scale, $r$. As we noted in section 2.2, this task is tackled with the bottomup approach, where the challenging aspect is to properly identify the relevant DOFs and symmetries at this scale. Accordingly, we start by considering in section 3.1 the addition of worldline coordinate DOFs, which enter the point-particle action in equation (2.9), and account for the simplified case of a non-rotating point mass coupled to gravity. 
Next, we proceed in section 3.2 to take into account the actual case of a rotating object coupled to gravity by adding rotational worldline DOFs to the point-particle action. As it turns out, the extension of the point-particle formulation to describe a spinning particle is quite challenging. This is due to the fundamental conflict between an actual rotating gravitating object, which must have an extended finite size for its rotational velocity to not surpass the speed of light, and its view in the EFT as a point particle.

Finally, dissipative effects, which occur at the small scale of the single object, such as the absorption of gravitational radiation on the horizon of BHs, or the dynamical tidal deformations of NSs, should also be taken into account. Therefore, in section 3.3 we discuss the addition of new worldline DOFs to the point-particle action, which are dissipative and hence dynamical.

\subsection{Point mass}

We consider first the basic case of a non-spinning massive object [39, 41]. In this case the only DOFs to add to the gravitational field modes are the worldline coordinates, $y^{\mu}$, and the relevant symmetries of the theory are the following:

1. General coordinate invariance, including parity invariance, where the latter plays a key role beyond minimal coupling;

\section{Worldline reparametrization invariance;}

3. Internal Lorentz invariance of the local frame field.

The latter is an additional gauge freedom of the tetrad field that covers the spacetime manifold, and it is further considered in the gauge choices discussed in section 4.1.

Let us first consider the minimal coupling in the point-particle action. Then we have the following term:

$$
\begin{aligned}
-m \int d \tau & =-m \int \sqrt{\bar{g}_{\mu \nu} d y^{\mu} d y^{\nu}} \\
& =-m \int d \sigma \sqrt{\bar{g}_{\mu \nu} \frac{d y^{\mu}}{d \sigma} \frac{d y^{\nu}}{d \sigma}}=-m \int d \sigma \sqrt{u^{2}}
\end{aligned}
$$

where $\tau$ is the proper time along the worldline, and $u^{\mu} \equiv \frac{d y^{\mu}}{d \sigma}$ is the coordinate velocity. This leading term includes the Newtonian interaction at the $0 \mathrm{PN}$ order, and provides higherorder PN corrections. Though usually the term 'point-mass' refers to the approximation, that consists only of this leading term, in the EFT context it refers to all terms in the action, which are induced by the presence of the mass, considered as a point particle.

Hence, we proceed to the non-minimal coupling terms in the point-particle action, namely to mass-induced higher multipoles, which account for finite size effects, where the internal structure of the objects starts to play a role. Considering operators with some dependence on the Riemann tensor and covariant derivatives, the first option that arises involves the Ricci tensor, $R_{\mu \nu}$. However, the leading EOMs of the gravitational field are sourceless, i.e. of a vacuum spacetime, so $R_{\mu \nu}=0$. Therefore, operators constructed with 
the Ricci tensor are what is referred to in EFT terminology as redundant operators, and can be practically omitted from the effective action. Formally, one can redefine the field in the operator, such that its Wilson coefficient is made to vanish. Suppose, for example, that we consider the operator $c_{R} \int d \tau R$. Then we can redefine the metric, which also appears in the Einstein-Hilbert term, according to $g_{\mu \nu} \rightarrow g_{\mu \nu}+\delta g_{\mu \nu}$, as follows:

$$
\delta g_{\mu \nu}=-16 \pi G \cdot \epsilon_{R} \int d \tau \frac{\delta^{d}(y-y(\tau))}{\sqrt{g}} g_{\mu \nu}
$$

where $\epsilon_{R}$ is some unfixed coefficient. Plugging this redefinition of the field into the gravitational action results in a shift of the Wilson coefficient, $c_{R} \rightarrow c_{R}+\epsilon_{R}$, so by adjusting $\epsilon_{R}$ we can make $c_{R}$ vanish. This well-known property in EFTs is analogous to the well-known application of leading EOMs of coordinates in higher-order perturbative Lagrangians, e.g. in PN ones, which is also found to be formally equivalent to the redefinition of coordinates, as is further discussed in detail in section 4.3. The property, that operators, which vanish 'on-shell', can be omitted from the effective action, is very general, and it is repeatedly used throughout the construction of the point-particle action.

Subsequently, we consider operators that contain the Riemann tensor. The curvature tensor is thus decomposed into its electric and magnetic components of definite parity, which are defined as follows:

$$
\begin{aligned}
& E_{\mu \nu} \equiv R_{\mu \alpha \nu \beta} u^{\alpha} u^{\beta}, \\
& B_{\mu \nu} \equiv \frac{1}{2} \epsilon_{\alpha \beta \gamma \mu} R_{\delta \nu}^{\alpha \beta} u^{\gamma} u^{\delta},
\end{aligned}
$$

that is of even and odd parity, respectively. These tensors are usually defined with the Weyl tensor, which due to the Ricci flatness is equivalent in our case to the Riemann tensor. The leading mass-induced higher-order operators are thus quadratic in the Riemann tensor, such that the point-particle action takes the following form:

$$
S_{\mathrm{pp}}=-m \int d \sigma \sqrt{u^{2}}+c_{E} \int d \sigma \frac{E_{\mu \nu}^{2}\left(y^{\alpha}(\sigma)\right)}{\left[\sqrt{u^{2}}\right]^{3}}+c_{B} \int d \sigma \frac{B_{\mu \nu}^{2}\left(y^{\alpha}(\sigma)\right)}{\left[\sqrt{u^{2}}\right]^{3}} .
$$

These new operators stand for the mass-induced quadrupolar tidal deformation of the extended object by the gravitational field. Each added derivative in an operator scales as $1 / r$, and is preceded by a Wilson coefficient with the proper power of $r_{s}$, so that overall each term scales as powers of $r_{s} / r=v^{2}$. Thus, from dimensional analysis one easily finds that the leading Wilson coefficients $c_{E, B}$ in equation (3.5), which are equivalent to the 'Love numbers' originally defined in Newtonian gravity, scale as $r_{s}^{5}$ at LO, and that these finite size operators enter at the $5 \mathrm{PN}$ order. For NSs however, $r_{s}^{5} \gg(G m)^{5}$, so the PN scaling breaks down, and the numerical value of the Wilson coefficients of tidal couplings can be large. This is also discussed in section 3.3, where dissipative effects at the scale of the single object are considered.

More generally, an effective action for mass-induced tidal deformations has been constructed in [56]. Furthermore, the electric Love numbers of static BHs, including the $c_{E}$ 
coefficient in equation (3.5), have been studied in the EFT framework in an arbitrary dimension in [57]. There, it was found that these Wilson coefficients, including $c_{E}$, vanish for BHs at $d=4$, where $d$ is the number of spacetime dimensions. Note that these coefficients are gauge invariant, though their matching is always done in some specific gauge, which implies that when they vanish, they vanish at all scales, and there is no RG running of this coupling. Interestingly, it was also found in [57], that at higher dimensions, i.e. at $d>4$, these coupling constants do not vanish, and that for a half-integral value of $\frac{l}{d-3}$, where $l$ is the multipole exponent, e.g. for the case of $c_{E(d=7)}$, the couplings exhibit a (classical) RG flow, consistent with the divergences of the one-particle EFT.

All in all, we conclude that for the non-spinning case of a massive particle the effective action is given by equation (3.5) up to the $5 \mathrm{PN}$ order.

\subsection{Spinning particle}

Let us now proceed to consider the actual, real case of a spinning gravitating object, which is much more challenging to handle. Unlike the mass of the object, which is a feature readily compatible with the EFT perspective of a point particle, incorporating the spin of the object into the EFT framework is far from evident. This is essentially since a spinning object in relativity must be an extended one, i.e. it has a non-vanishing finite size, with the lower bound for its size being the radius of the ring singularity of a Kerr BH, below which the rotational velocity of the spinning object would have exceeded the speed of light. This conflict with the point-particle perspective is actually related to the fundamental problem of how to define a sensible 'center' of an object in relativistic physics, since the unique notion of the 'center of mass' becomes ambiguous once we leave Newtonian physics.

Thus, there is in fact in this case an important additional gauge freedom, that should be carefully taken into account: the choice of an internal point within the extended object, whose trajectory along the linear motion is to be followed, and which is to designate the evolving position of the spinning object. Worldline tetrads, as well as tetrad fields equivalent to the metric field, need to be invoked to describe the rotation of the object in curved spacetime. We define all multipoles of the single object beyond the mass monopole, namely its spin dipole and higher spin-induced multipoles, coupled to the gravitational field, through the rotating DOFs of the object. As was explicitly shown in [75], the choice of the timelike vector of the worldline tetrad of the object is equivalent to the abovementioned choice of the internal point within the object, and in turn equivalent to the choice of the spin variable of the object.

Therefore, from an EFT viewpoint, there are additional DOFs and related symmetries, that need to be properly identified and correctly considered in order to construct the effective action, beyond those mentioned in section 3.1 for the point-mass case. First, it is assumed that the isolated object has no intrinsic permanent multipole moments beyond the spin dipole. Then, for the DOFs related with the rotation of the object, which are discussed in detail in what follows, the following symmetries are formulated [75]:

1. $S O(3)$ rotational invariance of the worldline spatial triad; 
2. Spin gauge invariance, which is an invariance under the choice of a completion to a tetrad of the worldline spatial triad through a timelike vector.

The action for a spinning particle in relativity was first considered decades ago in both flat and curved spacetimes, e.g. in [122] and [123], respectively, though not within an EFT framework. It was first tackled with an EFT approach in [61] in order to reproduce PN effects at LO, and to approach effects at NLO, linear in the spins of the objects [62]. To that end, a Routhian approach from [124], devised for up to the quadratic order in the spin, was later adopted in $[63,65]$ in order to produce the EOMs of the objects at NLO.

An independent application within the EFT approach was made in [64, 68] for spin effects up to NLO, where it was explicitly noted that the spin gauge can be fixed at the level of the one-particle EFT, and then one would be left only with the physical $S O(3)$ rotational DOFs. Following these works, and the first treatment of spin effects at NNLO within the EFT approach in [69], further formal clarifications for a proper treatment of spin within an EFT framework were made in [70], and an EFT for a spinning particle was presented in [75]. The latter enabled a proper rederivation of all the spin effects in the conservative sector up to NLO, the obtainment of further effects up to the quartic order in the spin [73], and the completion of the effects up to the quadratic order in the spins at NNLO $[71,72,74]$. These constitute the current state of the art in the conservative sector with spins, namely at the level of the EFT of the composite particle, where the additional ingredients that make up the advances at this subsequent level are the topic of section 4.

It should be noted that yet another independent formulation to handle arbitrary gravitating spinning objects, based on a coset construction for spacetime symmetries, was put forward in [77]. The effective action developed in the latter contains only physical rotational DOFs. However, this action is an expansion in $v_{\text {rot }} / c_{s}$, where $v_{\text {rot }}$ is the rotational velocity, and $c_{s}$, the speed of sound, which scales as $c_{s} \sim c$ for relativistic matter. For this reason this formulation is ideal for slowly rotating objects, i.e. whenever the angular frequency is smaller than the characteristic frequencies of the object, such as for NSs. This formulation was applied to tackle dissipative effects, which are discussed in section 3.3, for spinning objects in an EFT approach in [81, 82], though these effects were not specified for the PN context.

Let us then proceed to outline the construction of the effective action of a spinning particle starting from the minimal coupling part, as already formulated in [122, 123], and pressing on to the formal EFT developments provided in $[75,76]$ for both the minimal and non-minimal coupling parts of the effective action.

\subsubsection{Minimal coupling}

We start by adding to the worldline DOFs of the single object the tetrad, $e_{A}^{\mu}(\sigma)$, an orthonormal set consisting of a timelike future-oriented vector and three spacelike vectors, which satisfies $\eta^{A B} e_{A}^{\mu}(\sigma) e_{B}{ }^{\nu}(\sigma)=g^{\mu \nu}$. From the coordinate of the object, $y^{\mu}(\sigma)$, we already have the four-velocity, $u^{\mu} \equiv \frac{d y^{\mu}}{d \sigma}$, and from the tetrad we further define the angular velocity tensor, $\Omega^{\mu \nu} \equiv e_{A}^{\mu} \frac{D e^{A \nu}}{D \sigma}$, which generalizes the flat spacetime definition, $\Omega^{a b} \equiv$ $\Lambda_{A}^{a} \frac{d \Lambda^{A b}}{d \sigma}$, with Lorentz transformations, $\Lambda_{A}^{a}$. 
Since the action is reparametrization invariant, the Lagrangian is required to be a homogeneous function of degree 1 in the velocities $u^{\mu}$ and $\Omega^{\mu \nu}$. Therefore if we define the linear momentum, $p_{\mu}$, and the antisymmetric spin tensor, $S_{\mu \nu}$, as follows:

$$
\begin{aligned}
p_{\mu} & \equiv-\frac{\partial L_{\mathrm{pp}}}{\partial u^{\mu}}, \\
S_{\mu \nu} & \equiv-2 \frac{\partial L_{\mathrm{pp}}}{\partial \Omega^{\mu \nu}},
\end{aligned}
$$

where the sign is fixed according to the NR limit, then from Euler's theorem we get the following form for the minimal coupling part of the action [122, 123]:

$$
L_{\mathrm{pp}}=-p_{\mu} u^{\mu}-\frac{1}{2} S_{\mu \nu} \Omega^{\mu \nu}
$$

where the linear momentum satisfies $p^{\mu}=m \frac{u^{\mu}}{\sqrt{u^{2}}}+\mathcal{O}\left(S^{2}\right)$, independent of the gauge chosen for the rotational DOFs, $e_{A}^{\mu}(\sigma)$. In fact, a unique covariant gauge, which eliminates the unphysical DOFs from the rotational DOFs, was provided by Tulczyjew in [125]. This gauge reads as follows:

$$
e_{0}^{\mu}=\frac{p^{\mu}}{\sqrt{p^{2}}}
$$

which corresponds to what is referred to as a 'spin supplementary condition' (SSC), given by

$$
S_{\mu \nu} p^{\nu}=0,
$$

so we see that this unique gauge fixes the spin to be a spatial $S O(3)$ tensor in the body-fixed tetrad frame.

Therefore, we highlight that the spin is considered as a further dynamical worldline DOF, which serves as a classical source from a QFT perspective. Hence, the starting point for the whole action of a spinning particle reads as follows [75]:

$$
S_{\mathrm{pp}}\left[\bar{g}_{\mu \nu}\left(y^{\mu}\right), u^{\mu}, e_{A}^{\mu}, S_{\mu \nu}\right](\sigma)=\int d \sigma\left[-m \sqrt{u^{2}}-\frac{1}{2} S_{\mu \nu} \Omega^{\mu \nu}+L_{\mathrm{NMC}}\left[\bar{g}_{\mu \nu}\left(y^{\mu}\right), u^{\mu}, S_{\mu \nu}\right]\right],
$$

where the label 'NMC' here refers to the non-minimal coupling part of the action induced by the presence of spin, and the covariant gauge is implied.

First, we would like to restore to the action the gauge freedom of the rotational variables [75], in the spirit of the Stueckelberg action; see, e.g. [126]. Rather than resorting to changing the gauge of the spin variable at a later stage, we apply a generic transformation to the worldline tetrad in order to make the action manifestly gauge invariant to the choice of gauge of rotational variables. We do this by applying an effectively covariant boost to the worldline tetrad, and then considering how the rotational minimal coupling term, $\frac{1}{2} S_{\mu \nu} \Omega^{\mu \nu}$, and the non-minimal coupling part of the action, are affected.

Using a boost in its four-dimensional covariant form, $L_{\nu}^{\mu}(w, q)$, we transform the tetrad, $e_{A}^{\mu}$, from some gauge $e_{0}^{\mu}=q^{\mu}$ to a generic gauge for the tetrad as follows:

$$
\hat{e}_{0}^{\mu}=w^{\mu}
$$


with the transformation $\hat{e}_{A}^{\mu}=L_{\nu}^{\mu}(w, q) e_{A}^{\nu}$. Then, starting from the covariant gauge, i.e. taking $q^{\mu}=\frac{p^{\mu}}{\sqrt{p^{2}}}$, the generic gauge for the tetrad in equation (3.12) satisfies the following generic SSC:

$$
\hat{S}^{\mu \nu}\left(p_{\nu}+\sqrt{p^{2}} \hat{e}_{0 \nu}\right)=0 .
$$

The generic spin variable, $\hat{S}^{\mu \nu}$, is then related to $S^{\mu \nu}$ by

$$
\hat{S}^{\mu \nu}=S^{\mu \nu}-\delta z^{\mu} p^{\nu}+\delta z^{\nu} p^{\mu}
$$

where $\delta z^{\mu} \equiv \hat{z}^{\mu}-y^{\mu}$, so that we see that indeed this gauge freedom also corresponds to a choice of an object's 'center', $\hat{z}$. Assuming then, that we started from the covariant gauge, we get the following for the minimal coupling term:

$$
\frac{1}{2} S_{\mu \nu} \Omega^{\mu \nu}=\frac{1}{2} \hat{S}_{\mu \nu} \hat{\Omega}^{\mu \nu}+\frac{\hat{S}_{\mu \nu} p^{\nu}}{p^{2}} \frac{D p^{\mu}}{D \sigma} .
$$

The new extra term on the RHS of equation (3.15) contributes to finite size effects with spin, yet it carries no Wilson coefficient. This is since this term does not encapsulate any UV physics of the structure of the object, rather it just accounts for the fact that a spinning object is of a finite size. An elaborate discussion of this extra term is found in [75].

We note that for a maximally rotating object, i.e. which rotates at the speed of light, the spin of the compact object scales as $S \sim m r_{s}$. With the spin being derivatively coupled, the leading PN spin contribution from equation (3.15) then enters at order $r_{s} / r$ relative to the Newtonian term in equation (3.1), i.e. at the $1 \mathrm{PN}$ order for a rapidly rotating compact object. We will see in section 4.2 that this term actually starts contributing at the leading spin-orbit interaction of the composite particle, which enters only at the $1.5 \mathrm{PN}$ order.

As for the effect of restoring gauge invariance to the action in equation (3.11) in its non-minimal coupling part (that we analyze in detail below), which depends only on the spin, $S_{\mu \nu}$, we only need to use the following transformation to the generic spin variable:

$$
S_{\mu \nu}=\hat{S}_{\mu \nu}-\frac{\hat{S}_{\mu \rho} p^{\rho} p_{\nu}}{p^{2}}+\frac{\hat{S}_{\nu \rho} p^{\rho} p_{\mu}}{p^{2}} .
$$

\subsubsection{Non-minimal coupling}

Let us then proceed to consider how to construct the non-minimal coupling part of the action of a spinning particle [75]. We begin by noting that parity invariance plays a key role in fixing the non-minimal couplings. Hence, in order to construct the spin-induced ones in terms of ingredients of definite parity, let us first define the spin vector, $S^{\mu}$, using the dual to the spin tensor, $* S_{\alpha \beta} \equiv \frac{1}{2} \epsilon_{\alpha \beta \mu \nu} S^{\mu \nu}$ :

$$
S^{\mu} \equiv * S_{\nu}^{\mu} \frac{p^{\nu}}{\sqrt{p^{2}}} \simeq * S_{\nu}^{\mu} \frac{u^{\nu}}{\sqrt{u^{2}}}
$$

From this spacelike vector the spin length, $S^{2}$, is defined as $S^{2} \equiv-S_{\mu} S^{\mu}=\frac{1}{2} S_{\mu \nu} S^{\mu \nu}$.

From the Cayley-Hamilton theorem higher powers of the spin tensor are expected to be dependent. Indeed, considering higher powers of the spin tensor in the sense of 
matrix multiplication, one finds that the minimal polynomial of the spin tensor is of order 3: $x(x+i S)(x-i S)=0$ (as both the spin vector, $S^{\mu}$, and the four-velocity, $u^{\mu}$, are eigenvectors of the spin tensor, corresponding to the degenerate eigenvalue 0). Since we also find that a contraction of two spin tensors is equivalent to the direct product of two spin vectors $S^{\alpha} S^{\beta}$ (when contracted with traceless tensors, which are also orthogonal to $u^{\mu}$ ), we conclude that in order to construct the higher order spin-induced operators, we should use direct products of the spin vector, $S^{\alpha}$, which will have alternating parity. The scalar of spin length, $S^{2}$, is absorbed in the mass parameter, and renormalizes the Wilson coefficients. Considered in the body-fixed frame, the spin-induced multipoles are $S O(3)$ irrep tensors, since we recall that we start from the covariant gauge, $e_{0}^{\mu} \simeq \frac{u^{\mu}}{\sqrt{u^{2}}}$, so that $e_{i}^{\mu} u_{\mu}=0$. It is thus inferred that the spin-induced multipoles are symmetric, traceless, and spatial constant tensors in the body-fixed frame.

The even and odd spin-induced higher multipoles then are coupled to the even and odd parity electric and magnetic curvature components, $E_{\mu \nu}$ and $B_{\mu \nu}$, from equations (3.3) and (3.4), respectively, and their covariant derivatives. Here, we consider only operators linear in the Riemann tensor, namely not taking into account dissipative tidal effects (these are discussed in section 3.3, and are found in general to contribute at higher PN orders). From the symmetries of the Riemann tensor, the first Bianchi identity, and the leading vacuum field solution, it is easy to find, that $E_{\mu \nu}$ and $B_{\mu \nu}$ are both symmetric, traceless, and orthogonal to $u^{\mu}$. Hence, like the spin-induced higher multipoles, these are $S O(3)$ tensors, that we consider in the body-fixed frame, where they are spatial.

The covariant derivatives are also projected to the body-fixed frame, $D_{i}=e_{i}^{\mu} D_{\mu}$, and the time derivatives, $D_{0} \simeq u^{\mu} D_{\mu} \equiv D / D \sigma$, can be ignored to linear order in the Riemann tensor [75]. The indices of the covariant derivatives are also symmetric among themselves, and with respect to the indices of the electric and magnetic curvature tensors. The latter symmetry can be deduced from the differential Bianchi identity, where one obtains the following, in analogy to Maxwell's equations:

$$
\begin{aligned}
\epsilon_{i k l} D_{k} E_{l j} & =\dot{B}_{i j}, \\
\epsilon_{i k l} D_{k} B_{l j} & =-\dot{E}_{i j},
\end{aligned}
$$

and as we noted above regarding the time derivatives, the RHS can then be taken as vanishing. From further contracting the last equations we also find that traces involving the covariant derivatives vanish, i.e.

$$
D_{i} E_{i j}=D_{i} B_{i j}=0
$$

and similarly

$$
\square E_{i j}=\square B_{i j}=0
$$

Therefore, all in all, the electric and magnetic curvature tensors and their covariant derivatives form $S O(3)$ tensors like the spin-induced higher multipoles to which they couple. 
Based on the above analysis we can write down the LO spin-induced non-minimal couplings to all orders in spin as follows:

$$
\begin{aligned}
L_{\mathrm{NMC}} & =\sum_{n=1}^{\infty} \frac{(-1)^{n}}{(2 n) !} \frac{C_{E S^{2 n}}}{m^{2 n-1}} D_{\mu_{2 n}} \cdots D_{\mu_{3}} \frac{E_{\mu_{1} \mu_{2}}}{\sqrt{u^{2}}} S^{\mu_{1}} S^{\mu_{2}} \cdots S^{\mu_{2 n-1}} S^{\mu_{2 n}} \\
& +\sum_{n=1}^{\infty} \frac{(-1)^{n}}{(2 n+1) !} \frac{C_{B S^{2 n+1}}}{m^{2 n}} D_{\mu_{2 n+1}} \cdots D_{\mu_{3}} \frac{B_{\mu_{1} \mu_{2}}}{\sqrt{u^{2}}} S^{\mu_{1}} S^{\mu_{2}} \cdots S^{\mu_{2 n}} S^{\mu_{2 n+1}},
\end{aligned}
$$

with new spin-induced Wilson coefficients, which are identified as unity for Kerr BHs, but should in fact be properly matched using the full UV theory.

In particular, let us extract from the general sum in equation (3.22) the three leading terms, which contribute to finite size effects, due to the spin-induced quadrupole, octupole, and hexadecapole, given by the following, respectively

$$
\begin{aligned}
L_{E S^{2}} & =-\frac{C_{E S^{2}}}{2 m} \frac{E_{\mu \nu}}{\sqrt{u^{2}}} S^{\mu} S^{\nu}, \\
L_{B S^{3}} & =-\frac{C_{B S^{3}}}{6 m^{2}} D_{\lambda} \frac{B_{\mu \nu}}{\sqrt{u^{2}}} S^{\mu} S^{\nu} S^{\lambda}, \\
L_{E S^{4}} & =\frac{C_{E S^{4}}}{24 m^{3}} D_{\lambda} D_{\kappa} \frac{E_{\mu \nu}}{\sqrt{u^{2}}} S^{\mu} S^{\nu} S^{\lambda} S^{\kappa} .
\end{aligned}
$$

Again, it is easy to see that for each spin-induced higher multipole a spin vector (divided by a mass factor) is added along with an additional derivative. Hence, for a maximally rotating compact object the additional relative factor scales as $r_{s} / r$, and thus the quadrupole, octupole, and hexadecapole, enter at the $2 \mathrm{PN}, 3 \mathrm{PN}$, and $4 \mathrm{PN}$ orders, respectively. However, similar to what we noted above for the odd parity spin dipole, and as we will see in section 4.2 , the odd parity spin-induced octupole starts contributing at the leading spin-induced octupole-orbit interaction of the composite particle, which actually enters only at the 3.5PN order.

To conclude, we see that spin-induced finite size effects significantly dominate over finite size effects, which are induced by the mass.

\subsection{Dissipative DOFs}

In order to capture dissipative effects, which take place at the scales of the single object, such as the absorption of gravitational energy by the horizons of BHs, or the dissipative tidal deformations of NSs, further worldline DOFs should be added to the EFT beyond the coordinate and rotational DOFs [78]. In principle the complete theory would also contain a part that specifies the theory of the microscopic DOFs associated with the dissipation. However, even if the underlying detailed nature of these DOFs is unknown, it is possible to use the symmetries of spacetime and of the object to construct the related infinite set of operators of non-minimal coupling in the point-particle action.

For BHs these DOFs would be localized on the horizon to account for horizon absorption effects, which is related to fundamental questions on the nature of the $\mathrm{BH}$ horizon 
and the microphysics of $\mathrm{BH}$ entropy. To begin with, using the $S O(3)$ symmetry of a static spacetime, the couplings of the horizon modes to gravity read as follows [78]:

$$
S_{\mathrm{pp}(\mathrm{diss})}=-\int d \tau Q_{a b}^{E} E^{a b}-\int d \tau Q_{a b}^{B} B^{a b}+\cdots,
$$

where $E^{a b}, B^{a b}$, are the electric and magnetic curvature components from equations (3.3) and (3.4), respectively, projected onto the locally flat frames, and $Q_{a b}^{E}, Q_{a b}^{B}$, are quadrupolar composite operators formed in some manner from the horizon DOFs. We note that it was suggested in [80] to decompose the metric field at this stage into three (rather than two) components of Fourier modes, $g_{\mu \nu}=g_{\mu \nu}^{s}+g_{\mu \nu}^{\text {hor }}+\bar{g}_{\mu \nu}$, where the additional component, $g_{\mu \nu}^{\text {hor }}$, represents the low frequency modes of the field at the near horizon zone and is identified as the dissipative DOFs on the horizon.

According to the classical optical theorem (see, e.g. [127], or [117]) for the quantum counterpart, it turns out that the power loss of the composite binary system is related to the imaginary part of its effective action, as follows [39]:

$$
2 \operatorname{Im}\left[S_{\text {eff(comp) }}\right]=\int d t \int \frac{d \omega}{E(\omega)} \frac{d P}{d \omega},
$$

where $S_{\text {eff(comp) }}$ is the effective action of the composite particle from equations (2.12) and (2.13), on which we elaborate in sections 4 and 5 , and $d P / d \omega$ is the power spectrum observable. The power spectrum, $d P_{\text {abs }} / d \omega$, which gets absorbed by the single objects through their dissipative DOFs in equation (3.26), can be directly related to the absorption cross section, $\sigma_{\text {abs }}(\omega)$.

Let us see then how this relation transpires. Since $Q_{a b}^{E, B}$ are dissipative DOFs, they dynamically propagate along the worldline. Due to rotational invariance $\left\langle Q_{a b}^{E, B}\right\rangle$ vanishes; then the LO contribution of the worldline operators in equation (3.26) to the absorption cross section and to equation (3.27) involves the correlators of these operators. One should then consider the two-point function along the worldline, which can be written as follows:

$$
\int d \tau e^{-i \omega \tau}\left\langle T Q_{a b}^{E, B}(\tau) Q_{c d}^{E, B}(0)\right\rangle=-\frac{i}{2}\left[\delta_{a c} \delta_{b d}+\delta_{a d} \delta_{b c}-\frac{2}{3} \delta_{a b} \delta_{c d}\right] F(\omega) .
$$

Here, $F(\omega)$ is identified as the response function that encodes the propagator of the dissipative DOFs (namely it encodes aspects of strong gravity), and it arises in the graviton absorption cross section as follows [78]:

$$
\sigma_{\mathrm{abs}}(\omega)=16 \pi G \omega^{3} \operatorname{Im}[F(\omega)]
$$

which is also known from purely classical gravitational physics, such that the response function is found to be given by

$$
\operatorname{Im}[F(\omega)]=\frac{16}{45} G^{5} m^{6}|\omega| .
$$

Hence, the propagator in equation (3.28) also enters the leading dissipative interaction potential of the binary composite, and then from equation (3.27) the power loss due to the 
absorption on the horizon is found. From power counting the response function, recalling that $\omega \sim v / r$, and the operators in equation (3.26), it is easily found that this leading dissipative interaction potential enters only at the $6.5 \mathrm{PN}$ order. Having found the power loss, we can now infer a generic relation between the power spectrum and the cross section of the absorption, where the cross section can be explicitly computed given a model for the internal structure of the single compact objects.

The generalization of [78] for absorption by rotating BHs was approached in [79]. For rotating $\mathrm{BHs}$, superradiance (the radiation enhancement due to interaction with a rotating object) yields the leading PN dissipative effect, which was reproduced in [79], and is enhanced by $v^{-3}$ with respect to the non-rotating case, namely entering at the $5 \mathrm{PN}$ order. Dissipative effects for arbitrary gravitating rotating objects were treated more generally in [81] and [82], following the effective action developed in [77], while also adding dissipative DOFs. In this approach, which followed [77], the tidal distortions and superradiance of rotating objects are both governed by the same dissipative couplings, and furthermore the matching procedure in the non-rotating case also holds with spin.

To treat dynamical tidal deformations of NSs, in particular resonances in non-rotating extended objects, a similar approach to [78] was invoked in [83-85], which captures effects such as tidal heating, tidal disruption, tidal locking, and resonances in bound binaries. The worldline action is supplemented with gravitational multipoles as dissipative DOFs, for which the dynamics is encoded in the propagator, i.e some response function. However, for generic stars the tower of effective theories, presented in section 2.2, actually starts from also having a fluid description of matter, that is, a theory for the matter should be added to equation (2.8), e.g. an ideal fluid action. For idealized Newtonian stars [84], and approximately also for stars in GR [83], the scale of the object can then be explicitly integrated out. This leads to a point-mass action augmented by harmonic oscillator DOFs, corresponding to oscillation modes of the star, which gives rise to resonances. Other dynamical tidal deformations are described by terms in the worldline action such as $\left(\dot{E}_{a b}\right)^{2} \equiv\left(u^{\mu} D_{\mu} E_{a b}\right)^{2}$.

\section{EFT of a composite particle}

In this section we discuss in detail the obtainment of the EFT of the composite particle, as defined in equation (2.12), namely via the top-down procedure, using standard perturbative QFT methods, involving a diagrammatic expansion and Feynman calculus. This EFT removes the scale of orbital separation between the components of the binary, $r$. It is then matched onto the effective action constructed bottom-up, as presented in equation (2.13), with the point-particle action now being that of the composite particle, in terms of multipole moments. More precisely, in this section we focus on the conservative sector, where one is concerned with the computation of two-particle interactions, which make up the mass/energy monopole of the composite particle. We also discuss how to work out standard results and observables from these two-body interactions. The matching from the full two-particle theory at the orbital scale onto the effective theory of the composite particle with multipole moments, involving the radiative sector, is discussed in the beginning of section 5.1. 
We open in section 4.1 by describing all the choices, and gauges, that are made in order to facilitate computation. Then, in section 4.2 we detail and review the Feynman rules (which are extracted from the two-particle theory in equation (2.11)), the Feynman diagrams corresponding to various interaction sectors, and the main issues in the Feynman calculus required for their evaluation. We review the buildup of these computations tackled in the works $[39,48,51-55,128,129]$ for the two-particle point-mass sector, which involves only the non-minimal coupling from section 3.1, from the 1PN order to the recently attained state of the art at the 4PN order. We note here related works, which have discussed further aspects of tackling the computations [130, 131], or their interpretation [132]. Moreover, we note the progress made in the computations in the two-particle sector with spins from section 3.2, which involve both minimal and non-minimal couplings, in the works [61-76], where the state of the art in PN theory was actually pushed forward.

Finally, in section 4.3 we discuss how to process the outcome of integrating out the orbital modes for the EFT of the composite particle in the conservative sector. In order to bring the effective action into a standard form, higher-order time derivatives should be handled. EOMs should be derived for the positions and spins of the constituents of the binary. Moreover, the Hamiltonian, which is the crucial input for the EOB framework, as well as other conserved integrals of motion, should be derived. The developments in the EFT formulation, computation, and their outcome, as well as further gauge invariant relations, e.g. among the binding energy of the binary and its total angular momentum, have been all put into a public code, dubbed 'EFTofPNG', in [112]. We elaborate on this public PN code later in section 5.2.

It should be stressed in particular for the sector including spins, that the resulting effective action should contain no remaining potential field DOFs [64, 68, 70, 75]. The observation that this key aspect of an EFT should be upheld was crucial in order to formally and computationally advance the state of the art in this sector, and it pertains to having a clear identification and separation between field and particle DOFs. Hence, to prepare for this EFT at the next stage, where we get the two-particle interactions by explicitly integrating over the orbital field modes, we need to disentangle the field DOFs from the particle DOFs, and to fix all relevant rotational gauges.

First, we have for the worldline tetrad the following factorization:

$$
\hat{e}_{A}^{\mu}=\hat{\Lambda}_{A}^{b} \tilde{e}_{b}^{\mu}
$$

so that the worldline tetrad is decomposed into rotational particle DOFs and field DOFs, namely the Lorentz matrices in the locally flat frames, defined by $\eta^{A B} \hat{\Lambda}_{A}^{a} \hat{\Lambda}_{B}^{b}=\eta^{a b}$, and the tetrad field, which covers the manifold, defined by $\eta_{a b} \tilde{e}_{\mu}^{a} \tilde{e}_{\nu}^{b}=g_{\mu \nu}$, respectively. For the latter an additional internal Lorentz invariance should be noted. Then, we rewrite the minimal coupling rotational term in terms of the new DOFs as follows [68]:

$$
\frac{1}{2} \hat{S}_{\mu \nu} \hat{\Omega}^{\mu \nu}=\frac{1}{2} \hat{S}_{a b} \hat{\Omega}_{\mathrm{LF}}^{a b}+\frac{1}{2} \hat{S}_{a b} \omega_{\mu}^{a b} u^{\mu}
$$

where $\hat{\Omega}_{\mathrm{LF}}^{a b} \equiv \hat{\Lambda}^{a A} \frac{d \hat{\Lambda}_{A}^{b}}{d \sigma}$ is the locally flat angular velocity, and $\omega_{\mu}^{a b} \equiv \tilde{e}_{\nu}^{b} D_{\mu} \tilde{e}^{a \nu}$ are the 
Ricci rotation coefficients, so that the eventual particle rotational variables are the Lorentz matrices, $\hat{\Lambda}_{A}^{a}$, and the spin, $\hat{S}_{a b}$, in the locally flat frame.

Our generic gauge from equations (3.12) and (3.13), for the rotational variables then reads as follows [75]:

$$
\begin{aligned}
\hat{\Lambda}_{0}^{a} & =w^{a}, \\
\hat{S}^{a b}\left(p_{b}+\sqrt{p^{2}} \hat{\Lambda}_{0 b}\right) & =0 .
\end{aligned}
$$

Therefore, the separation of field and particle DOFs is not yet complete, since $w^{a}=\tilde{e}_{\mu}^{a} w^{\mu}$, and the temporal spin components, $\hat{S}^{0 i}$, contain further field dependence. It is, in fact, not surprising from the EFT viewpoint that specifying the point-particle action necessitates fixing the gauge of rotational variables at the level of the one-particle action. This is due to the fact that only once this choice is made is the view of the extended object as a point particle actualized. Hence, only once the gauge of the rotational variables is fixed, can the field be completely disentangled from the particle DOFs, and all orbital field modes can be explicitly integrated out, as required at this stage [64, 70, 75].

\subsection{KK metric and gauge fixing}

First, let us recall from equation (2.10) that for the orbital field modes we have $k_{0} \simeq v / r$, whereas $|\vec{k}| \simeq 1 / r$, so that for the propagator in momentum space, given by

$$
\int \frac{d^{4} k}{(2 \pi)^{4}} e^{-i k x} \frac{1}{k^{2}}=\int \frac{d^{4} k}{(2 \pi)^{4}} e^{-i k_{0} t+i \vec{k} \cdot \vec{x}} \frac{1}{k_{0}^{2}-\vec{k}^{2}},
$$

the denominator can be expanded in the PN approximation as follows [41]:

$$
\frac{1}{k_{0}^{2}-\vec{k}^{2}}=-\frac{1}{\vec{k}^{2}}\left(1+\frac{k_{0}^{2}}{\vec{k}^{2}}+\cdots\right)=-\frac{1}{\vec{k}^{2}}\left(1+\mathcal{O}\left(v^{2}\right)\right),
$$

and we get the following for the propagator of the orbital component of the field:

$$
\int \frac{d k_{0}}{2 \pi} e^{-i k_{0} t} \int \frac{d^{3} \vec{k}}{(2 \pi)^{3}} \frac{e^{i \vec{k} \cdot \vec{x}}}{\vec{k}^{2}}=\delta(t) \int \frac{d^{3} \vec{k}}{(2 \pi)^{3}} \frac{e^{i \vec{k} \cdot \vec{x}}}{\vec{k}^{2}},
$$

so that the propagator is instantaneous, and the relativistic time corrections to the propagator are considered as quadratic perturbations [41].

Thus, in the context of the NR regime of gravity it is useful to switch to a unique space+time parametrization of the metric, since in the NR limit the time dimension can be regarded as compact in comparison to the spatial dimensions $[48,50]$. Therefore it makes sense to reduce over the time coordinate in the metric in a Kaluza-Klein (KK) fashion, so that the metric is rewritten as follows:

$$
d s^{2}=g_{\mu \nu} d x^{\mu} d x^{\nu} \equiv e^{2 \phi}\left(d t-A_{i} d x^{i}\right)^{2}-e^{-2 \phi} \gamma_{i j} d x^{i} d x^{j},
$$

which defines the KK fields: $\phi, A_{i}$, and $\gamma_{i j} \equiv \delta_{i j}+\sigma_{i j}$, identified as the Newtonian scalar, the gravito-magnetic vector, and the tensor fields, respectively, where $\gamma^{i j} \gamma_{j k} \equiv \delta_{k}^{i}$, and $A^{i} \equiv \gamma^{i j} A_{j}{ }^{10}$.

\footnotetext{
${ }^{10}$ We note that an exponential parametrization of the metric coefficients was also introduced in [133].
} 
Let us then proceed to fix the gauge of the various DOFs. For the purely gravitational action, $S_{g}$, the (fully) harmonic gauge is chosen, so that the action reads as follows:

$$
\begin{aligned}
S_{g} & =S_{E H}+S_{G F} \\
& =-\frac{1}{16 \pi G} \int d^{4} x \sqrt{g} R+\frac{1}{32 \pi G} \int d^{4} x \sqrt{g} g_{\mu \nu} \Gamma^{\mu} \Gamma^{\nu},
\end{aligned}
$$

where $\Gamma^{\mu} \equiv \Gamma_{\rho \sigma}^{\mu} g^{\rho \sigma}$. Working within the background field method, on which we elaborate in section 5.1, covariant derivatives are taken with respect to the background field [39, 117]. However, in the conservative sector that we are now considering, no background radiation modes are present, and so the covariant derivative is just the standard one, i.e. taken with respect to the asymptotic flat spacetime. Using Cartan's method of two-forms, the full gravitational action can be computed in terms of the KK parametrization [49], and the propagators and self-interaction vertices, which we present in section 4.2, are readily obtained from the action in this parametrization.

We still have to fix the internal Lorentz gauge of the tetrad field, which couples to the worldline multipoles. Again, in the NR regime, where the time direction is singled out, it is convenient to choose Schwinger's time gauge [134], which reads as follows:

$$
\tilde{e}_{i}^{0}(x)=0,
$$

so that in terms of the KK fields from equation (4.8), the tetrad field reads as follows [75]:

$$
\tilde{e}^{a}{ }_{\mu}=\left(\begin{array}{cc}
e^{\phi} & -e^{\phi} A_{i} \\
0 & e^{-\phi} \sqrt{\gamma}_{i j}
\end{array}\right)
$$

where $\sqrt{\gamma_{i j}}$ is the symmetric square root of $\gamma_{i j}$.

For the worldline parameter we choose the time coordinate, $t=y^{0}$, such that $\sigma=t$, $u^{0}=1$, and $u^{i} \equiv \frac{d y^{i}}{d t}=v^{i}$. Hence in terms of the KK metric in equation (4.8) the mass coupling in equation (3.1) is rewritten as follows:

$$
-m \int d t \sqrt{g_{\mu \nu} \frac{d x^{\mu}}{d t} \frac{d x^{\nu}}{d t}}=-m \int d t\left[e^{\phi} \sqrt{\left(1-A_{i} v^{i}\right)^{2}-e^{-4 \phi} \gamma_{i j} v^{i} v^{j}}\right],
$$

which is then expanded in the velocity, $v$, and the KK fields.

Finally, for the rotational variables we recall that one starts by assuming the covariant gauge, namely $\hat{\Lambda}_{0 a}=\frac{p_{a}}{\sqrt{p^{2}}}$ for the Lorentz matrix; however, it is found to be beneficial to switch to the 'canonical gauge' [75]. The latter is the general relativistic generalization of what is known as the Pryce-Newton-Wigner SSC in special relativity [135, 136], and it reads as follows:

$$
\begin{aligned}
\hat{\Lambda}_{0}^{a} & =\delta_{0}^{a}, \\
\Longrightarrow \hat{S}^{a b}\left(p_{b}+\sqrt{p^{2}} \delta_{0 b}\right) & =0 .
\end{aligned}
$$

This gauge enables one to completely integrate out the orbital field modes as required at this stage, and leads to physical spin variables, $\hat{S}^{i j}$, which eventually satisfy canonical Poisson brackets, as we further discuss in section 4.3 . 


\subsection{Feynman graphs and calculus}

After we applied a beneficial parametrization for the metric and fixed all gauges in the previous section, we can proceed to extract the Feynman rules from the gauge-fixed twoparticle effective action. These rules constitute the building blocks of the Feynman graphs, which make up the perturbative expansion of the exponent in the functional integral in equation (2.12). The Feynman rules can also be generated automatically in a comprehensive manner using the 'EFTofPNG' public code, on which we elaborate in section 5.2. All Feynman rules are given in position space in what follows.

Following from equation (4.7) we start by extracting the propagators from the quadratic time-independent part of the gravitational action in terms of the KK metric. The propagators of the KK fields then read as follows:

$$
\begin{aligned}
& \left\langle\phi\left(x_{1}\right) \phi\left(x_{2}\right)\right\rangle==\quad 4 \pi G \times \delta\left(t_{1}-t_{2}\right) \int_{\vec{k}} \frac{e^{i \vec{k} \cdot\left(\vec{x}_{1}-\vec{x}_{2}\right)}}{\vec{k}^{2}}, \\
& \left\langle A_{i}\left(x_{1}\right) A_{j}\left(x_{2}\right)\right\rangle=-\cdots-16 \pi G \times \delta\left(t_{1}-t_{2}\right) \int_{\vec{k}} \frac{e^{i \vec{k} \cdot\left(\vec{x}_{1}-\vec{x}_{2}\right)}}{\vec{k}^{2}} \delta_{i j}, \\
& \left\langle\sigma_{i j}\left(x_{1}\right) \sigma_{k l}\left(x_{2}\right)\right\rangle=\overline{=}=32 \pi G \times \delta\left(t_{1}-t_{2}\right) \int_{\vec{k}} \frac{e^{i \vec{k} \cdot\left(\vec{x}_{1}-\vec{x}_{2}\right)}}{\vec{k}^{2}} P_{i j ; k l},
\end{aligned}
$$

where we abbreviate $\int \frac{d^{3} \vec{k}}{(2 \pi)^{3}}$ as $\int_{\vec{k}}$, and $P_{i j ; k l} \equiv \frac{1}{2}\left(\delta_{i k} \delta_{j l}+\delta_{i l} \delta_{j k}-2 \delta_{i j} \delta_{k l}\right)$. Hence, the KK decomposition allows one to use simplified propagators, which reduce the overload of tensor indices. For the relativistic time correction vertices on the propagators from equation (4.6) we then have the following:

$$
\begin{aligned}
\square & =\frac{1}{8 \pi G} \int d^{4} x\left(\partial_{t} \phi\right)^{2}, \\
--\times-\cdots & =-\frac{1}{32 \pi G} \int d^{4} x\left(\partial_{t} A_{i}\right)^{2}, \\
\overline{\times} & =\frac{1}{128 \pi G} \int d^{4} x\left[2\left(\partial_{t} \sigma_{i j}\right)^{2}-\left(\partial_{t} \sigma_{i i}\right)^{2}\right],
\end{aligned}
$$

where the crosses represent these quadratic self-gravitational vertices, containing two time derivatives. However, in GR there are also non-linear self-interaction vertices, which give rise to loop integrals, where $n$-graviton vertices correspond to $(n-2)$ loops. For example, the following cubic vertices can be easily read from the static part of the gravitational action in equation (4.9) in terms of the KK parametrization:

$$
\begin{aligned}
& =\frac{1}{16 \pi G} \int d^{4} x\left[2 \sigma_{i j} \partial_{i} \phi \partial_{j} \phi-\sigma_{i i} \partial_{j} \phi \partial_{j} \phi\right], \\
& =\frac{1}{8 \pi G} \int d^{4} x \phi\left[\partial_{i} A_{j}\left(\partial_{i} A_{j}-\partial_{j} A_{i}\right)+\left(\partial_{i} A_{i}\right)^{2}\right] .
\end{aligned}
$$

Next, we proceed to consider the worldline graviton couplings, which arise from the point-particle action. From equation (4.12) we get the following one-graviton couplings for the mass couplings of the objects:

$$
\downarrow \quad=-m \int d t \phi\left[1+\frac{3}{2} v^{2}+\mathcal{O}\left(v^{4}\right)\right]
$$




$$
\begin{aligned}
& \downarrow=m \int d t A_{i} v^{i}\left[1+\mathcal{O}\left(v^{2}\right)\right], \\
& \square=\frac{m}{2} \int d t \sigma_{i j} v^{i} v^{j}\left[1+\mathcal{O}\left(v^{2}\right)\right],
\end{aligned}
$$

where a bold vertical line represents a worldline, a mass coupling is represented by a black circle, and there is an infinite expansion in $v^{2}$. We can already see from these couplings one of the benefits of the KK decomposition, for example, in organizing the PN hierarchy of the coupling to the mass of the graviton field components. From further expanding equation (4.12) in the fields the $n$-graviton mass couplings are obtained, e.g. the following leading two-graviton mass coupling:

$$
\mathcal{L}=-\frac{1}{2} m \int d t \phi^{2}\left[1+\mathcal{O}\left(v^{2}\right)\right]
$$

For the spin couplings from equations (3.15) and (4.2), which originate from the minimal coupling, it should be stressed first, that just as the mass sector contains kinetic terms with no field couplings (see equation (4.12)), then there are also kinematic contributions involving spin, given by

$$
L_{\mathrm{pp}[\mathrm{S}(\mathrm{kin})]}=-\vec{S} \cdot \vec{\Omega}+\frac{1}{2} \vec{S} \cdot \vec{v} \times \vec{a}\left(1+\frac{3}{4} v^{2}+\mathcal{O}\left(v^{4}\right)\right),
$$

where $a^{i} \equiv \dot{v}^{i}$, and all indices are Euclidean. We also recall that at this stage there are only physical spin and angular velocity components, namely $S_{i} \equiv \frac{1}{2} \epsilon_{i j k} S_{j k}, \Omega_{i} \equiv \frac{1}{2} \epsilon_{i j k} \Omega_{j k}$, where $\epsilon_{i j k}$ is the three-dimensional Levi-Civita symbol, and $\Omega_{i j} \equiv-\Lambda_{i k} \dot{\Lambda}_{k j}$, where the Lorentz matrices are now the $S O(3)$ rotation matrices. Then, there are the following one-graviton spin couplings, e.g.:

$$
\left\{\begin{array}{l}
=\int d t \epsilon_{i j k} S_{k}\left(\frac{1}{2} \partial_{i} A_{j}+\frac{3}{4} v^{i} v^{l}\left(\partial_{l} A_{j}-\partial_{j} A_{l}\right)+v^{i} \partial_{t} A_{j}+\cdots\right), \\
=\int d t 2 \epsilon_{i j k} S_{k}\left(v^{i} \partial_{j} \phi+\cdots\right)
\end{array}\right.
$$

where the gray oval represents a spin coupling, and the ellipsis stands for higher PN order terms.

Proceeding to the non-minimal coupling, we get from equation (3.23), for example, the following one-graviton spin-squared coupling:

$$
\downarrow=\int d t\left[\frac{C_{E S^{2}}}{2 m} S^{i} S^{j} \partial_{i} \partial_{j} \phi+\cdots\right]
$$

where the black square box represents the spin-squared quadrupole. From equation (3.24) we get, for example, the following couplings to the spin-induced octupole:

$$
\begin{aligned}
& \downarrow=\int d t\left[-\frac{C_{\mathrm{BS}^{3}}}{12 m^{2}} S^{i} S^{j} \epsilon_{k l m} S^{m} \partial_{i} \partial_{j} \partial_{k} A_{l}+\cdots\right], \\
& \square=\int d t\left[\frac{C_{\mathrm{BS}^{3}}}{3 m^{2}} S^{i} S^{j} \epsilon_{k l m} S^{m} \partial_{i} \partial_{j} \partial_{k} \phi v^{l}+\cdots\right],
\end{aligned}
$$




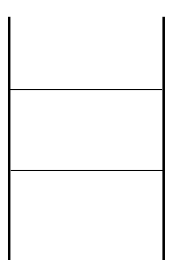

(a)

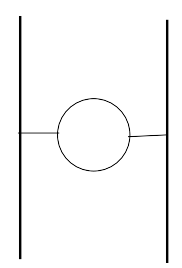

(b)

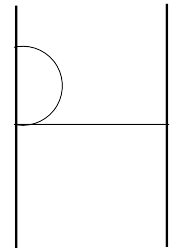

(c)

Figure 4. Graph topologies that are excluded from the diagrammatic expansion of the two-particle interaction for the EFT of the composite particle [39, 41]: (a) Graph with more than a single connectivity component (where worldlines are stripped off); (b) Graph containing a graviton loop; (c) Graph renormalizing the UV divergence related with the Wilson coefficients of the one-particle EFT, e.g. the mass. We note that the bold vertical lines represent the worldlines, where time flows up, rather than from left to right as is customary in particle physics.

where the gray rectangular boxes represent the octupole. Finally, from equation (3.25) the coupling to the quartic spin hexadecapole reads as follows:

$$
\downarrow=\int d t\left[-\frac{C_{\mathrm{ES}}{ }^{4}}{24 m^{3}} S^{i} S^{j} S^{k} S^{l} \partial_{i} \partial_{j} \partial_{k} \partial_{l} \phi+\cdots\right],
$$

where the black crossed box represents the hexadecapole.

We are now in a position to consider the construction of the Feynman graphs, which contribute to the various two-particle interactions [39, 41]. All such graphs would contain the two worldlines of the components of the binary, and gravitons, which propagate and mediate the interaction between them. In addition, the worldlines are to be interchanged, as the interaction is symmetric under the exchange of the two particles. Next, we note that there are some graph topologies that will be excluded from the diagrammatic expansion, as in the examples displayed in figure 4: 1 . Graphs that contain more than a single connectivity component (where worldlines are stripped off the graph), since by definition the effective action is the exponent in equation (2.12); 2. Graphs that contain graviton loops (i.e. again, worldlines stripped off), as we are concerned here with classical gravity, and hence only tree graphs are relevant; 3. Graphs that UV renormalize the Wilson coefficients of the one-particle EFT, i.e. the mass, spin, or the coefficients of the higher induced multipoles ${ }^{11}$. We note that in figure 4 and henceforth, graphs show the time flowing up in accordance with the way spacetime is drawn in relativity (unlike in figure 3, or in many works in the field, where time flows from left to right, according to the convention in particle physics).

Next, let us consider the bare graph topologies at each order of the gravitational constant, $G$. To this end we consider the generic power counting of the fields as inferred from the Feynman rules for the propagators, where at first each $n$-graviton self-interaction vertex scales as $G^{\frac{n}{2}-1}$, and each $n$-graviton worldline coupling scales as $G^{\frac{n}{2}}$ [51]. Therefore at the order of $G^{1}$ there is only a single possible topology of a one-graviton exchange, as can be seen in figure 5. At the order of $G^{2}$ there are two possible topologies, where there is already one topology with a cubic self-interaction, namely a one-loop graph, as can be

\footnotetext{
${ }^{11}$ These power-divergent diagrams are set to zero in dimensional regularization.
} 


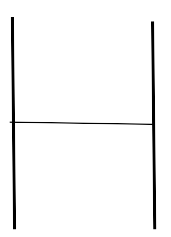

Figure 5. The single topology at $\mathcal{O}\left(G^{1}\right)$ : One-graviton exchange with no gravitational selfinteraction.

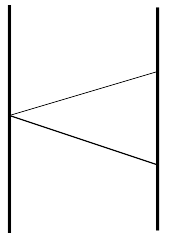

(a)

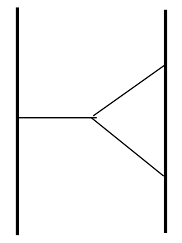

(b)

Figure 6. The topologies at $\mathcal{O}\left(G^{2}\right)$ : (a) Two-graviton exchange; (b) Single cubic self-interaction. This is a one-loop topology. When using the KK metric only topology (a) enters at the 1PN order [48]; see figure 7. Both topologies enter at the $2 \mathrm{PN}$ order of the point-mass sector [51].

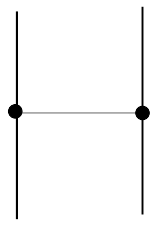

(a0)

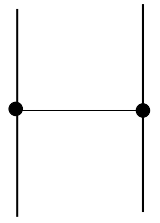

(a)

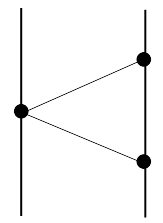

(d)

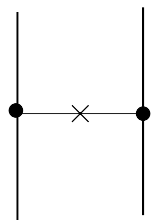

(b)

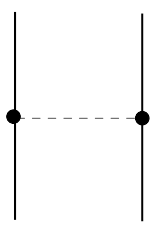

(c)

Figure 7. The Newtonian (0PN) and first post-Newtonian (1PN) order interactions [39, 48]: (a0) The Newtonian interaction is mediated by the scalar field, $\phi$, of the KK metric; (a)-(c) At the 1PN order we start to take into account higher-order mass couplings, as in (a) and (c), and the relativistic correction to the instantaneous propagator in (b); (d) At the non-linear level we expect in general to also have a one-loop graph [39], but with the KK metric it is deferred to the next PN order, and there is only a single diagram of two-graviton exchange [48, 51].

seen in figure 6. Generally, at the order of $G^{n}$, which corresponds to the $(n-1) \mathrm{PN}$ order, we first encounter $(n-1)$-loop topologies. 
We then proceed to dress these bare topologies with actual vertices from the Feynman rules, that are $\mathrm{PN}$-weighed according to their power in $v$. The evaluation of the resulting Feynman diagrams involves the usual Wick contractions, symmetry factors, and Feynman integrals, familiar from QFT. For example, the Newtonian interaction, which appears in figure $7(\mathrm{a} 0)$, is evaluated as follows:

$$
\text { Figure } \begin{aligned}
7(\mathrm{a} 0) & =-m_{1} \int d t_{1} \phi\left(y_{1}\right) \times\left(-m_{2}\right) \int d t_{2} \phi\left(y_{2}\right) \\
& =4 \pi G \times m_{1} m_{2} \int d t_{1} d t_{2} \delta\left(t_{1}-t_{2}\right) \int_{\vec{k}} \frac{e^{i \vec{k} \cdot\left(\vec{y}_{1}\left(t_{1}\right)-\vec{y}_{2}\left(t_{2}\right)\right)}}{\vec{k}^{2}} \\
& =\int d t \frac{G m_{1} m_{2}}{r},
\end{aligned}
$$

where $r \equiv|\vec{r}| \equiv\left|\vec{y}_{1}-\vec{y}_{2}\right|$, and the action of the familiar Newtonian interaction is obtained. After Wick contracting the scalar fields using the propagator in equation (4.15), we integrate over time with the delta function, and for the Fourier integral the following scalar master integral is needed:

$$
I \equiv \int \frac{d^{d} \vec{k}}{(2 \pi)^{d}} \frac{e^{i \vec{k} \cdot \vec{r}}}{\left(\vec{k}^{2}\right)^{\alpha}}=\frac{1}{(4 \pi)^{\frac{d}{2}}} \frac{\Gamma\left(\frac{d}{2}-\alpha\right)}{\Gamma(\alpha)}\left(\frac{r^{2}}{4}\right)^{\alpha-\frac{d}{2}}
$$

where dimensional regularization is always used throughout, and eventually the limit $d \rightarrow 3$ is taken. This master integral can be easily derived using Schwinger parameters [137]. In general, the master integral in equation (4.35) is required for all computations, where the related tensorial integrals are simply obtained by differentiating the scalar integral with respect to $\vec{r}$.

The diagrams that make up the 1PN order interaction are shown in figure $7(\mathrm{a})-(\mathrm{d})$, where the KK metric is used [48]. These contain higher-order mass couplings and the leading relativistic correction to the Newtonian instantaneous propagators. In general, a one-loop graph enters at the 1PN order [39, 128]. However, if the KK metric is used, a one-loop graph implies that all mass couplings must be of the scalar field, $\phi$, since only these couplings scale as $\sim v^{0}$, and the cubic self-interaction should be a static one, with only $\phi$ fields. However, in the KK metric the cubic vertex with $\phi$ fields is of the form $\phi\left(\partial_{t} \phi\right)^{2}$, i.e. it is time dependent, and since each time derivative scales as $v^{1}$, one-loop graphs are postponed to the $2 \mathrm{PN}$ order $[48,51]$. In general, the $n \mathrm{PN}$ order in the pointmass sector requires computation at the $n$-loop level, but using the KK metric only the $2\lfloor n / 2\rfloor$-loop level is required. For the sector with spins the $n$-loop computation is shifted to the $(n+1.5) \mathrm{PN}$ order or higher for rapidly rotating objects.

A crucial component in the evaluation of diagrams is the treatment of the time derivatives, which appear already at the 1PN order; see figure $7(\mathrm{~b})$. To this end the following generic identity is central:

$$
\int d t_{1} d t_{2} \partial_{t_{1}} \delta\left(t_{1}-t_{2}\right) f\left(t_{1}\right) g\left(t_{2}\right)=-\int d t_{1} d t_{2} \partial_{t_{2}} \delta\left(t_{1}-t_{2}\right) f\left(t_{1}\right) g\left(t_{2}\right)
$$




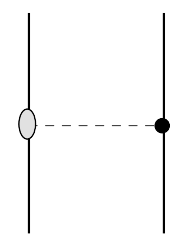

(a1)

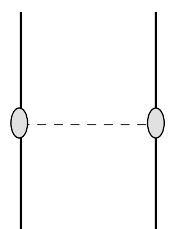

(b)

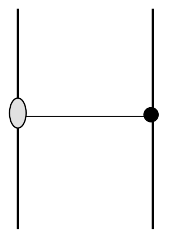

(a2)

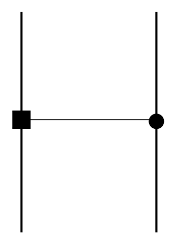

(c)

Figure 8. The LO PN interactions up to quadratic order in the spins: (a1)-(a2) The LO spin-orbit interaction. This is the leading PN correction with spins, which contributes at the $1.5 \mathrm{PN}$ order for rapidly rotating compact objects [61, 68, 75]; (b) The LO spin1-spin2 interaction between the spins of the two objects [61, 64]; (c) The LO spin-squared interaction, containing the spin-induced quadrupole of the object. This is the leading PN interaction from finite size effects, which contains a non-trivial Wilson coefficient that should be matched $[65,73]$. The interactions, which are quadratic in the spins, contribute at the $2 \mathrm{PN}$ order for rapidly rotating compact objects.

where repeated integration by parts (IBP) is used to drop the time derivatives on the worldline couplings. Each integral in position space coming from the bulk vertices transforms as usual into a delta function conserving momentum in the vertex, and for a one-loop integral the following scalar master integral is used:

$$
\begin{aligned}
J & \equiv \int \frac{d^{d \vec{k}}}{(2 \pi)^{d}} \frac{1}{\left[\vec{k}^{2}\right]^{\alpha}\left[(\vec{k}-\vec{q})^{2}\right]^{\beta}} \\
& =\frac{1}{(4 \pi)^{d / 2}} \frac{\Gamma(\alpha+\beta-d / 2)}{\Gamma(\alpha) \Gamma(\beta)} \frac{\Gamma(d / 2-\alpha) \Gamma(d / 2-\beta)}{\Gamma(d-\alpha-\beta)}\left(q^{2}\right)^{d / 2-\alpha-\beta} .
\end{aligned}
$$

This formula can easily be derived using Feynman and Schwinger parameters [137], and the related tensor integrals are similarly derived.

Let us continue and consider interactions involving the spins of the objects in the binary. These interactions enter as of the $1.5 \mathrm{PN}$ order for rapidly rotating objects, i.e. the next PN correction, after the $1 \mathrm{PN}$ order from the point-mass sector of figure 7 , with the spin-orbit interaction being the leading one in the sector with spins. The LO PN interactions up to quadratic order in the spins can be seen in figure $8[61,64,65,68,73,75]$. It is important to note that already at the LO spin-orbit interaction, shown in graphs (a1)(a2) of figure 8 , the gauge of the rotational variables needs to be addressed, unlike the subleading LO spin1-spin2 and spin-squared interactions, shown in graphs (b) and (c) of figure 8 , respectively, which contribute at the $2 \mathrm{PN}$ order for the case of rapidly rotating compact objects. Also noteworthy is that from the LO spin-orbit interaction (and from the $2 \mathrm{PN}$ order correction in the point-mass sector), one starts to encounter accelerations, and higher-order time derivatives, which should be properly removed via variable redefinitions, as we elaborate in section 4.3 . 


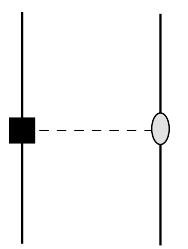

(a)

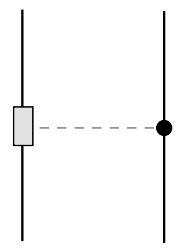

(c)

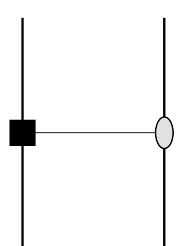

(b)

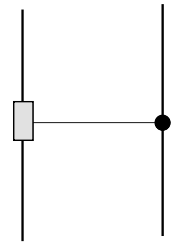

(d)

Figure 9. The LO interaction at cubic order in the spins, which contributes at the $3.5 \mathrm{PN}$ order for rapidly rotating compact objects $[73,75]$. This interaction is in analogy with the LO spinorbit interaction in figure 8(a1)-(a2) according to the parity of the spin-induced multipoles: (a)-(b) The spin-induced quadrupole interacting with the spin dipole; (c)-(d) The spin-induced octupole interacting with the mass monopole.

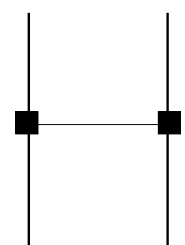

(a)

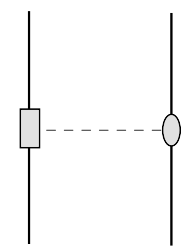

(b)

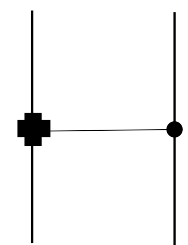

(c)

Figure 10. The LO interaction at quartic order in the spins, which contributes at the $4 \mathrm{PN}$ order for rapidly rotating compact objects [73, 75]: (a) and (c) Two spin-induced quadrupoles interacting and a spin-induced hexadecapole interacting with a mass monopole, respectively, both in analogy with the LO spin-squared interaction in figure 8(c); (b) Spin-induced octupole interacting with a spin dipole in analogy with the LO spin1-spin2 interaction in figure 8(b).

The leading non-trivial finite size effect is thus spin-induced, namely the LO spinsquared interaction with the spin-induced quadrupole in figure 8(c), and it is preceded by a Wilson coefficient, which encapsulates the internal physics of the compact object, and should be matched. Further spin-induced finite size effects at LO are shown in figures 9 and 10, corresponding to interactions at cubic and quartic order in the spins, respectively, also including the spin-induced octupoles and hexadecapoles [73, 75], and further Wilson coefficients to be matched. The interactions at cubic and quartic order in the spins contribute at the $3.5 \mathrm{PN}$ and $4 \mathrm{PN}$ orders, respectively, for rapidly rotating compact objects. It is easy to see the analogy of these interactions with those in figure 8 , which are lower in the order of the spins, corresponding to the parity of the spin-induced multipoles.

Let us proceed to the order of $G^{3}$ to gain more insight into the topologies and computations that arise at higher PN orders. The topologies at the order of $G^{3}$ are shown in figure $11[42,51]$. We encounter new topologies up to the one-loop level, which can be 


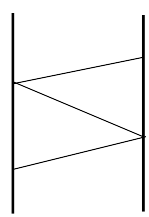

(a1)

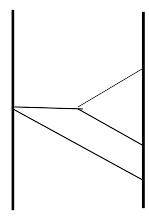

(b1)

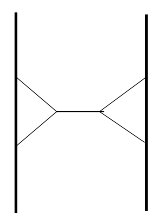

(c1)

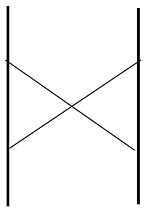

(d1)

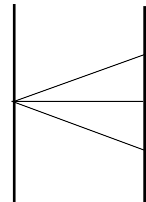

(a2)

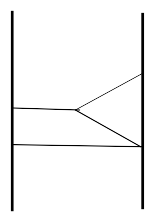

(b2)

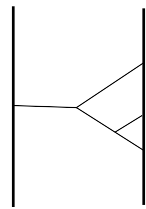

(c2)

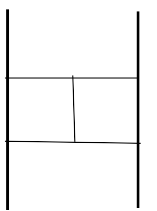

(c3)

Figure 11. The topologies at $\mathcal{O}\left(G^{3}\right)$ [51, 52]: (a) Up to three-graviton exchanges; (b) One cubic self-interaction with a two-graviton exchange; (c) Two cubic self-interactions; (d) One quartic selfinteraction. The graphs in (c) and (d) are the two-loop topologies. The analogy in the evaluation of graphs (c1) and (c2), with graphs (d1) and (d2), respectively, can be easily seen from the topologies. It can also be easily seen that graph (c1) is factorizable, i.e. it factorizes into 2 one-loop topologies, and that graph (c2) is nested, i.e. it consists of a one-loop topology nested within a one-loop topology. On the other hand, graph (c3) is not trivially reducible to one-loop topologies. When using the KK metric only the five topologies in graphs (a) and (c) enter at the $2 \mathrm{PN}$ order of the point-mass sector [51]. All of the topologies enter at the 3PN order [53].

clearly factorized into topologies that appeared in lower orders, as in figures 11(a1)-(b2), whereas figures 11(c1)-(d2) display two-loop topologies. Nevertheless, it can be easily seen that graphs (c1) and (d1) can be factorized into 2 one-loop topologies, and that in graphs (c2) and (d2) there is a one-loop topology nested within a one-loop topology. The topology, which appears in figure 11(c3) thus remains the only topology at this order that represents a new computational feature. Hence there is only one kind of two-loop integral, corresponding to graph (c3), which cannot be readily broken down into a one-loop computation $[51,69,130]$. In such topologies we encounter two-loop integrals, which contain products of five propagators that cannot be trivially disentangled.

However, these two-loop integrals can be reduced to a sum of factorizable and nested two-loop integrals [51, 69, 114], using the IBP method [137], which yields the following 


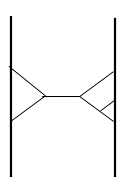

(a1)

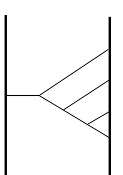

(a2)

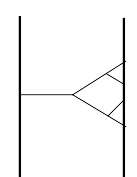

(a3)

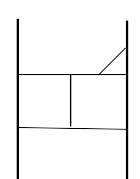

(a4)

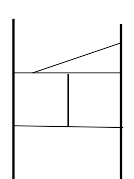

(a5)

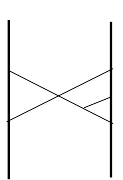

(b1)

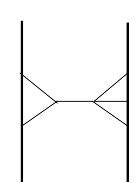

(b2)

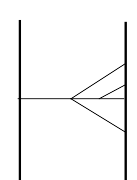

(b3)

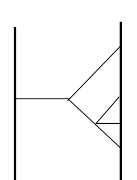

(b4)

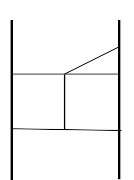

(b5)

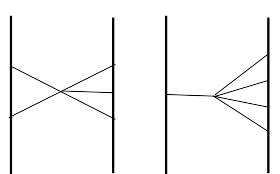

(c1)

(c2)

Figure 12. The three-loop topologies at $\mathcal{O}\left(G^{4}\right)$ (out of the total 32 topologies at this order): (a) Three cubic vertices; (b) One cubic vertex and one quartic vertex; (c) One quintic vertex. As in figure 11, it can be readily identified which topologies are factorizable and/or nested two-loop topologies. The few graphs, which are not trivially reduced to one-loop topologies, are reduced in a similar manner as in graph (c3) of figure 11, i.e. by using the IBP relation in equation (4.38) [114]. Regardless, when using the KK metric, none of these three-loop topologies enter at the 3PN order of the point-mass sector [53], but they all enter at the 4PN order of the point-mass sector [114].

useful reduction relation:

$$
\begin{aligned}
F\left(a_{1}, a_{2}, a_{3}, a_{4}, a_{5}\right) & \equiv \int_{\vec{k}_{1}, \vec{k}_{2}} \frac{1}{\left[\vec{k}_{1}^{2}\right]^{a_{1}}\left[\left(\vec{k}-\vec{k}_{1}\right)^{2}\right]^{a_{2}}\left[\vec{k}_{2}^{2}\right]^{a_{3}}\left[\left(\vec{k}-\vec{k}_{2}\right)^{2}\right]^{a_{4}}\left[\left(\vec{k}_{1}-\vec{k}_{2}\right)^{2}\right]^{a_{5}}} \\
& =\frac{a_{1}\left[F\left(a_{1}+, a_{3}-\right)-F\left(a_{1}+, a_{5}-\right)\right]+[1 \leftrightarrow 2,3 \leftrightarrow 4]}{a_{1}+a_{2}+2 a_{5}-d},
\end{aligned}
$$

where we use abbreviated notation, e.g. $F\left(a_{1}+, a_{3}-\right) \equiv F\left(a_{1}+1, a_{2}, a_{3}-1, a_{4}, a_{5}\right)$, and $1 \leftrightarrow 2$ is an exchange of the exponent labels. This reduction relation yields intermediate expressions with explicit poles in $d=3$, but these cancel out in the dimensional regularization. When using the KK metric only the five topologies in graphs (a) and (c) of figure 11 enter at the $2 \mathrm{PN}$ order of the point-mass sector [51]. All of the topologies at $\mathcal{O}\left(G^{3}\right)$ enter at the $3 \mathrm{PN}$ order [53].

As it turns out there are no new computational features that appear at $\mathcal{O}\left(G^{4}\right)$, and hence at the $3 \mathrm{PN}$ order. The most complex graphs at $\mathcal{O}\left(G^{4}\right)$ are the three-loop topologies, which are all shown in figure 12. As in $\mathcal{O}\left(G^{3}\right)$ in figure 11, the topologies that are factorizable and/or nested two-loop topologies are readily recognized. The few, which are not trivially reduced to one-loop topologies, are reduced similarly to graph (c3) of figure 11, i.e. by using the IBP relation in equation (4.38) [114]. Regardless, when using the KK metric, none of these three-loop topologies enter at the 3PN order of the point-mass sector [53], and they all enter only at the $4 \mathrm{PN}$ order of the point-mass sector [114]. Let us underline that notably thus far all of the topologies up to $\mathcal{O}\left(G^{4}\right)$, i.e. up to the three-loop 


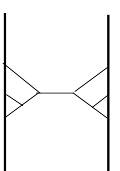

(a1)

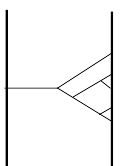

(a6)

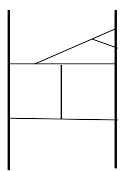

(a11)

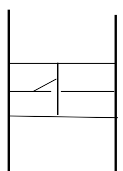

(a16)

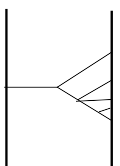

(b5)

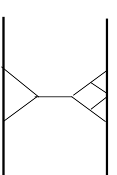

(a2)

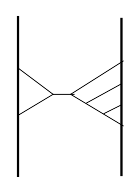

(a3)

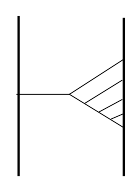

(a4)

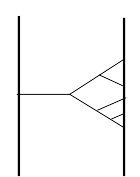

(a5)

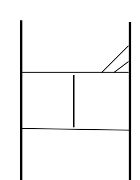

(a7)

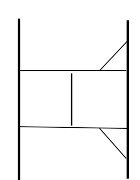

(a8)

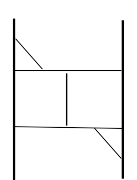

(a9)

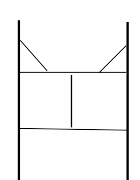

(a10)

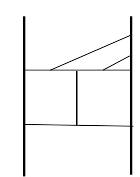

(a12)

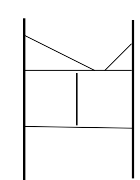

(a13)

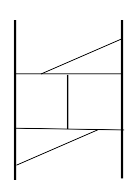

(a14)

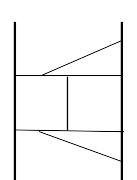

(a15)

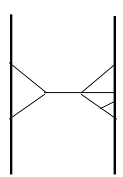

(b1)

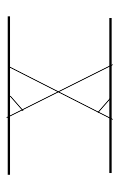

(b2)

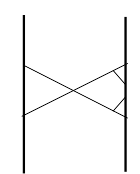

(b3)

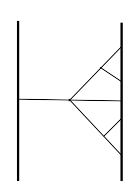

(b4)

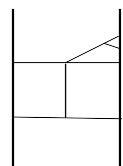

(b6)

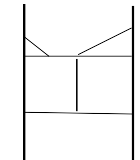

(b7)

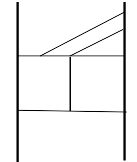

(b8)

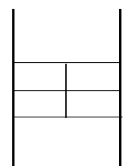

(b9)

Figure 13. The four-loop topologies at $\mathcal{O}\left(G^{5}\right)$, which enter at the $4 \mathrm{PN}$ order using the KK metric: (a) Four cubic vertices; (b) Two cubic vertices and one quartic vertex. As in figures 11 and 12, the topologies, which are factorizable and/or nested, are easily identified. It is also visibly recognizable that graphs (a9)-(a16) and (b7)-(b9) have a more complex structure to compute, whereas the few other graphs, which are not trivially reducible, are reduced to one-loop integrals using IBP [55]. The complex graphs require knowledge of certain four-loop master integrals in the limit $d \rightarrow 3$. Out of these, graphs (a16) and (b9) in particular, which are also represented as the two-point graphs in figure 14, require a specific four-loop master integral, which was evaluated in analytic form in the GR context in [55] and [129] independently.

level, reduce to one-loop integrals.

However, at the $4 \mathrm{PN}$ order, which involves four-loop topologies at $\mathcal{O}\left(G^{5}\right)$, four-loop master integrals are required $[55,114]$. The four-loop topologies at $\mathcal{O}\left(G^{5}\right)$, which enter at the $4 \mathrm{PN}$ order using the KK metric, are shown in figure 13. Since the computational complexity at this order goes considerably beyond the one-loop level, it is useful to bring up at this point an analogy between the graphs in our gravitational two-particle EFT and two-point functions of massless gauge theory in QFT [131]. Since our classical sources on the worldlines do not propagate, any graph at $\mathcal{O}\left(G^{n}\right)$ in our EFT can be mapped onto a 


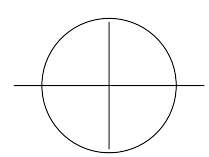

(a)

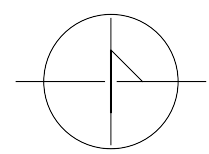

(b)

Figure 14. The four-loop two-point topologies at the 4PN order using the KK metric, corresponding to the topologies in figure 13, that contain the four-loop master integral represented by graph (a), which is evaluated in the GR context in analytic form in [55] and [129] independently: (a) Topology with a quartic vertex, corresponding to graph (b9) in figure 13; (b) Topology with only cubic vertices, corresponding to graph (a16) in figure 13.

$(n-1)$-loop two-point function with massless internal lines, or pictorially

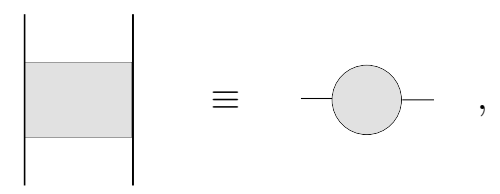

with external momentum $p, p^{2} \neq 0$, which is the momentum transfer of the source, i.e. just the Fourier transform momenta in the EFT. This analogy enables one to evaluate the graphs in figure 13, using standard QFT multiloop techniques based on IBPs, and known four-loop master integrals.

As in figures 11 and 12, the topologies in figure 13, which are factorizable and/or nested, are easily identified. It is also readily recognized, that graphs (a9)-(a16) and (b7)(b9) in figure 13 have a more complex structure, whereas the few other graphs, which are not trivially reducible, are reduced to one-loop integrals using IBP [55]. As for the complex graphs (a9)-(a16) and (b7)-(b9) in figure 13, these are reduced, using IBP technology, to linear combinations of seven specific four-loop master integrals [55]. Of these four-loop master integrals, one, which actually contributes in the $d \rightarrow 3$ limit, and is represented by the two-point graph (a) in figure 14, was reproduced in analytic form as an expansion in $d-3$, and evaluated in the GR context independently in [55] and [129]. Specifically, graphs (a16) and (b9) in figure 13, which are also represented as the two-point graphs in figure 14, require this particular four-loop master integral.

To conclude, the current state of the art in the conservative sector of the EFT of the composite particle is at the $4 \mathrm{PN}$ order. It has been recently tackled and completed in the point-mass sector in multiple works via various methods [30], including the ADM canonical formalism [138-141], the Fokker action [142, 143], and the EFT approach [54, 55, 129], where the works using EFT have presented partial results up to $\mathcal{O}\left(G^{2}\right)$, and at $\mathcal{O}\left(G^{5}\right)$. Ambiguities, which appeared between the results obtained via the ADM Hamiltonian approach, and intermediate results of the Fokker action approach, related to IR divergences, were further discussed in $[132,144]$. In addition to the graph topologies shown in this section in figures 5, 6, and 11-13, a contribution, that is non-local in time, which also enters at the conservative $4 \mathrm{PN}$ order, and originates from the leading non-linear radiation-reaction effect, is shown in graph (c) of figure 20. This effect involves interaction with radiation, and hence its discussion is deferred to section 5.1. 
On par with the accuracy attained in the non-spinning case, the $4 \mathrm{PN}$ order has also been completed in the conservative sector with spins, particularly with the completion of the full LO interaction, which is quartic in the spins, in [73], and of the NNLO spin-squared interaction in [71]. These interactions were exclusively derived via the EFT approach, and are based on [75], where the formal developments, which led up to this current state of the art with spins, were described in section 3.2. Rather than the strictly computational challenge in the point-mass sector, with spins the main hurdle lies at the formal obtainment of an effective theory for a spinning particle, as discussed in section 3.2, on which the computations with the two-particle theory rely. At the 4PN order the bump in the pointmass sector is in the high loop level required in the computations within the two-particle theory, essentially, in the leap from the one-loop level, practically sufficient for lower PN orders, to the four-loop level. Notably, contrary to the point-mass sector, where the minimal coupling of the one-particle theory is sufficient for such high PN order, with spins the finite size effects from spin-induced higher multipoles, originating from non-minimal coupling, have to also be taken into account.

\subsection{Standard action, EOMs, and potentials}

After the progression presented in section 3 and the current section is carried out, in particular with the spins of the objects, we obtain interaction potentials, which contain only physical DOFs, and higher-order time derivatives of the variables, similar to the outcome in the non-spinning sector. From this point on the action is handled in the standard manner in the PN context. Higher-order time derivatives can be eliminated via redefinitions of the position and spin variables already at the level of the action [75]. The EOMs of the position and spin variables are then directly obtained via a proper variation of the action [70, 75]. Furthermore, Hamiltonian potentials are derived in a straightforward manner with a standard Legendre transform of the position variables [75]. Alternatively, if higher-order time derivatives are not treated at the level of the action, one can also derive the EOMs first via a proper variation of a generalized action. In this case the higher-order time derivatives can be equivalently removed at the level of the EOMs, so that the EOMs are eventually well-defined [75].

Indeed, in the PN perturbative scheme the resulting action contains higher-order time derivatives, such as $\vec{a}_{1} \equiv \dot{\vec{v}}_{1}$. A common procedure to reduce the action to a standard one is to eliminate the higher-order time derivatives via their substitution, using EOMs of lower PN orders, at the level of the action. Such a substitution entails a redefinition of variables [145-148], which can easily be seen, considering that a small variable shift results in a variation of the action, which exactly yields the EOM of the variable. In fact, the same idea applies in any perturbative context, and we note the analogy to the concept of redundant operators in EFTs [70], which vanish on-shell, as was also noted in section 3.1. Let us review the procedure for eliminating higher-order time derivatives of position variables, and its extension for a spin variable, which was provided in [70].

A shift of the position, $\Delta x$, adds to the Lagrangian the EOM multiplied by $\Delta x$, where higher orders in the shift are neglected. Thus, it is clear that we can set the shift to cancel terms with acceleration in the Lagrangian. This is precisely what we would get from 
substituting the EOM directly, with the notable subtlety, that there are also contributions, which are non-linear in the shift, $\Delta L\left(\mathcal{O}(\Delta x)^{2}\right)$. These contributions will enter at higher perturbative orders, and thus one should keep track of them. For further higher-order time derivatives the procedure should be iterated until all higher-order time derivatives are eliminated. As for the rotational variables, the Lorentz rotation matrix, $\Lambda^{i j}$, is transformed with a generator of rotations, $\omega^{i j}$, and the redefinitions of the spin variables are specified so as to rotate similarly to the rotation matrices. Then, similarly as with the position variables, we can fix the rotation generator to cancel the terms with a time derivative of the spin, $\dot{S}^{i j}$, so that the shift in the Lagrangian is just what we would get from substituting in the spin EOMs, up to higher-order contributions in the rotation generator. Again, one should keep a record of these non-linear contributions, which will affect higher PN orders.

For the EOMs of the spin variables, we should consider the spin-dependent part of the full action, recalling the rotational kinetic term [68], so that the action has the following form:

$$
S_{\mathrm{eff}(\mathrm{comp})}=\int d t\left[\sum_{a=1}^{2} \frac{m_{a}}{2} v_{a}^{2}-\sum_{a=1}^{2} \frac{1}{2}\left[S_{a}\right]_{i j}\left[\Omega_{a}\right]_{i j}-V\left(\vec{x}_{a}, \dot{\vec{x}}_{a}, \ddot{\vec{x}}_{a}, \ldots,\left[S_{a}\right]_{i j},\left[\dot{S}_{a}\right]_{i j}, \ldots\right)\right] .
$$

Such a form for the action is associated with a canonical so(3) algebra for the physical spin variable. Varying this action independently with respect to the spin, and to its conjugate, the angular velocity, the following EOMs for the three-dimensional physical spin variable are obtained:

$$
\dot{S}_{a}^{i j}=-4 S_{a}^{k[i} \delta^{j] l} \frac{\delta \int d t V}{\delta S_{a}^{k l}}=-4 S_{a}^{k[i} \delta^{j] l}\left[\frac{\partial V}{\partial S_{a}^{k l}}-\frac{d}{d t} \frac{\partial V}{\partial \dot{S}_{a}^{k l}}+\ldots\right] .
$$

Therefore, similar to the EOMs for the position, the EOMs for the spin should be obtained from a variation of the action, in terms of which the EFT is naturally formulated [70, 75].

After time derivatives of the spin (and velocity) have been removed, the EOMs of the spin take on the following simple form:

$$
\dot{S}_{a}^{i j}=-4 S_{a}^{k[i} \delta^{j] l} \frac{\partial V_{s}}{\partial S_{a}^{k l}},
$$

where $V_{s}$ denotes the potential reduced to standard form, and this is a restricted case of equation (4.41). If we impose on the spin variables the equal-time Poisson brackets, namely

$$
\left\{S_{a}^{i j}, S_{a}^{k l}\right\}=S_{a}^{i k} \delta^{j l}-S_{a}^{i l} \delta^{j k}+S_{a}^{j l} \delta^{i k}-S_{a}^{j k} \delta^{i l},
$$

then we can rewrite the EOMs in equation (4.42) in the following form:

$$
\dot{S}_{a}^{i j}=\left\{S_{a}^{i j},-V_{s}\right\}
$$

Therefore, after the elimination of time derivatives of the spin, one arrives at canonical spin variables.

Finally, the conservative action of the composite particle is invariant under global Poincaré transformations. This invariance gives rise to conserved integrals of motion (see, 
e.g. in [72]), including the Hamiltonian, which is crucially important as an input for the EOB framework. Since the higher-order time derivatives have been eliminated, we can perform a standard Legendre transform with respect to the position variables to obtain the Hamiltonian directly from the effective interaction potential. Related to that we note that physically equivalent Hamiltonians are obtained using canonical transformations [149], similar to the addition of total time derivatives to obtain equivalent Lagrangians.

\section{$5 \quad$ Effective theory of dynamical multipoles}

In this section we are concerned with the final stage of our EFT scheme, dealing with effects that take place at the radiation level, and completing the connection of the whole EFT framework to GW emission observables.

In section 5.1 we start by considering the EFT of the composite particle in the radiative sector, i.e. where radiation modes are present, which was first treated in detail in [86]. As the bottom-up approach was taken to set up the theory in equation (2.13), we first discuss in detail the matching of the multipole moments, i.e. the Wilson coefficients, of the EFT of the composite particle at the radiation scale from the full two-particle theory at the orbital scale. We then present hereditary tail effects, which were reproduced up to subleading corrections in [86]. The various singularities which arise in these tail effects are noted, of which a classical RG flow of the effective theory emerges for the mass quadrupole.

We take time to introduce further advanced QFT concepts and tools, required at this stage, as the radiating system is dissipative, and time reversal no longer holds for the EFT in the radiative sector. Thus we present the closed time path (CTP) formalism [150-152], and then we can proceed, via the top-down approach, to integrate out the radiation modes from the CTP effective action. We go on to present the analysis of the RG flow of the total mass of the system as it was recovered in [92], which is related with the leading tail effect and is also manifested in the leading non-linear radiation reaction. Thus, the progress made within the EFT approach in the treatment of the radiation reaction, which was initiated in $[99,100]$, is then presented.

We end in section 5.2 with an overview of the 'EFTofPNG' code, which constitutes the first public comprehensive automatization of PN theory, and also covers the whole of the EFT framework, as outlined in this review, from section 3 to the current section. The code provides high precision computation for PN inspirals, so as to ultimately serve as an accessible pipeline to GW observables for the diverse gravity community, and in particular the GW community.

\subsection{Radiation, radiation reaction, and RG flows}

The EFT of the composite particle in the presence of radiation modes was first discussed in [39, 41], and formulated in [86], using the bottom-up approach. The latter work studied tail effects, which constitute radiative PN corrections, up to the subleading ones, and the related divergences that arise. This analysis led to the first demonstration of a classical RG flow in the theory, of the mass quadrupole. Moreover, the matching of the theory at the radiation scale, i.e. of the multipole moments, to the full two-particle theory at the orbital 
scale was formulated and displayed up to NLO, and the effect of the tails on the emitted power was also derived. Furthermore, in [87] a general multipole expansion at the level of the action provided general expressions for the mass and current multipole moments, the radiated power, and the gravitational waveform amplitude.

Let us proceed to outline the primary developments made in [86]. We begin by once again building on the bottom-up approach presented in equation (2.13). We assume that the orbital separation has been integrated out, and can no longer be resolved, and we write down an effective action, which contains the appropriate DOFs and satisfies the proper symmetries at the radiation scale. This theory is actually similar to that which we encountered in the one-particle EFT in section 3, only that the remaining field modes at this stage are the radiation modes, and we now assume higher-order multipole moments, $l \geq 2$, which are sourcing the radiation. In fact, as our system is radiating, time reversal is lost, and later on we address this crucial point for the obtainment of the proper effective action. For now we start by further specifying the theory from equation (2.13).

We recall that the effective action of the single composite object coupled to the gravitational radiation field, $\widetilde{g}_{\mu \nu} \equiv \eta_{\mu \nu}+\widetilde{h}_{\mu \nu}$, reads [86, 87]:

$$
S_{\text {eff(comp) }}\left[\widetilde{g}_{\mu \nu}, y_{c}^{\mu}, e_{c A}^{\mu}\right]=-\frac{1}{16 \pi G} \int d^{4} x \sqrt{\widetilde{g}} R\left[\widetilde{g}_{\mu \nu}\right]+S_{\mathrm{pp}(\mathrm{comp})}\left[\widetilde{g}_{\mu \nu}\left(y_{c}\right), y_{c}^{\mu}, e_{c A}^{\mu}\right]\left(\sigma_{c}\right),
$$

where $S_{\mathrm{pp}(\mathrm{comp})}$ is the effective worldline action at the radiation scale, describing the composite object with some 'center of the object' coordinate, $y_{c}^{\mu}\left(\sigma_{c}\right)$, and tetrad, $e_{c A}^{\mu}\left(\sigma_{c}\right)$. The worldline action of the composite particle is then of the following form $[68,86,87]$ :

$$
\begin{aligned}
S_{\mathrm{pp}(\mathrm{comp})}\left[\widetilde{h}_{\mu \nu}, y_{c}^{\mu}, e_{c A}^{\mu}\right](t)=-\int d t \sqrt{\widetilde{g}_{00}}[ & M(t)+\frac{1}{2} \epsilon_{i j k} L^{k}(t)\left(\Omega_{\mathrm{LF}}^{i j}+\omega_{\mu}^{i j} u^{\mu}\right) \\
& -\sum_{l=2}^{\infty}\left(\frac{1}{l !} I^{L}(t) \nabla_{L-2} E_{i_{l-1} i_{l}}\right. \\
& \left.\left.-\frac{2 l}{(l+1) !} J^{L}(t) \nabla_{L-2} B_{i_{l-1} i_{l}}\right)\right],
\end{aligned}
$$

where also here the worldline is parametrized in terms of the time coordinate, $t . M$ here is the total mass of the composite object, and the spin connection, $\omega_{\mu}^{i j}$, couples here to the total angular momentum, $L^{i j} \equiv \epsilon_{i j k} L^{k}$, of the composite object. The $S O(3)$ tensors $I^{L}$ and $J^{L}$, with the superscript $L$ as an abbreviated notation for $i_{1} \cdots i_{l}(l \geq 2)$, which are symmetric and trace free (STF) with respect to the spatial Euclidean metric, are commonly referred to as the mass and current multipoles, and are of even and odd parity, respectively.

These moments constitute the full set of higher multipole moments of the composite object, similar to the induced higher multipoles in the non-minimal coupling in the effective action for a spinning particle, constructed in sections 3.1 and 3.2. However, since these DOFs, localized on the worldline, encode the internal physics of the composite particle, which yields the dissipative radiation, similar to the dissipative DOFs in section 3.3, they are dynamical. In particular, the $l=0,2$, multipole moments of the composite system, i.e. the total mass, $M(t)$, and the mass quadrupole, $I_{i j}(t)$, are the first two 'Wilson coefficients' of the theory which exhibit classical RG flows, related with well-known tail effects, 
as discussed below. As the sources of gravitational radiation, the multipole moments are coupled to the radiation modes, $\widetilde{h}_{\mu \nu}$, where $E_{i_{l-1} i_{l}}, B_{i_{l} i_{l+1}}$, are as in equations (3.3) and (3.4), the electric and magnetic projections of the Riemann tensor onto its even and odd parity components, respectively.

At this stage we are concerned with expectation values, and hence we proceed to consider an effective action for the composite particle, which is a functional of the radiation mode, regarded as a mean field, serving as a fixed background, using the background field method [39, 117, 152]. In this case the potential modes play the role of the fluctuating 'quantum' field, as the integration variable of the functional integral in the computation of the effective action, and the radiation modes serve as the 'classical' fixed background. The background field method reflects the spirit of Wilson's idea of integrating out the high momentum DOFs, while taking proper care to preserve the gauge invariance of the remaining low momentum modes, when we compute the effective action for a slowly varying background field. Therefore, the gauge-fixing in the background field method is modified in the radiative sector with respect to the conservative sector. Thus, in equation (4.9) covariant derivatives with respect to the background radiation field are used; this preserves the coordinate invariance with respect to the radiation field. The gauge-fixing condition is covariant with respect to the background field, so that when we functionally integrate over the fluctuating field to compute the effective action, the result still has general covariance of the background field.

Let us briefly illustrate what the reserved term 'effective action' used here actually designates in order to avoid ambiguity with the various effective actions, synonymous with effective theories, which are implied throughout [117]. Let us recall, for example, that for a QFT of a scalar field, $\phi$, in the presence of an external source, $J$, the generating functional, $Z[J]$, is defined as follows:

$$
\begin{aligned}
Z[J] & \equiv \exp ^{i W[J]} \\
& \equiv \int \mathcal{D} \phi \exp \left[i \int d^{4} x[\mathcal{L}[\phi]+J(x) \phi(x)]\right]
\end{aligned}
$$

where $Z[J]$ is the basic object of the functional formalism, and is the generating functional of correlation functions, and $W \equiv-i \log Z$ is the generating functional of connected correlation functions. Thus, the functional derivative of the exponent with respect to the external source yields the one-point function of the field, as follows:

$$
\frac{\delta}{\delta J(x)} W[J]=\langle\phi(x)\rangle \equiv \phi_{\text {mean }}(x)
$$

where we define the one-point function as a mean field. Now, we can proceed to obtain the exact one-point function, $\langle\phi\rangle$, in the full quantum theory as the minimum of some function, as if our field is still only classical. We just define the Legendre transform of $W[J]$ as follows:

$$
\Gamma\left[\phi_{\text {mean }}\right] \equiv W[J]-\int d^{4} y J(y) \phi_{\text {mean }}(y)
$$


where $\Gamma\left[\phi_{\text {mean }}\right]$ is known as the 'effective action', which satisfies

$$
\frac{\delta}{\delta \phi_{\text {mean }}(x)} \Gamma\left[\phi_{\text {mean }}\right]=-J(x),
$$

and then if the external source is set to vanish, the effective action satisfies

$$
\frac{\delta}{\delta \phi_{\text {mean }}(x)} \Gamma\left[\phi_{\text {mean }}\right]=0 .
$$

Then, the solutions to this equation are the values of the exact one-point function, $\langle\phi(x)\rangle$, in the full theory.

Now, in order to match for the EFT of the composite particle with multipole moments of equations (5.1) and (5.2), the specific form of the multipole moments should be computed, given the details of the short distance gravitational physics of the binary system, which makes up the internal structure of the composite particle [86]. This is a standard procedure in dealing with EFTs, and is carried out explicitly in this case. In order to do the matching, it is sufficient to consider the single graviton emission amplitudes in the full theory at the orbital scale. These amplitudes arise in terms of the following term in the effective action:

$$
\Gamma\left[\widetilde{h}_{\mu \nu}\right]=-\frac{1}{2} \int d^{4} x T^{\mu \nu} \widetilde{h}_{\mu \nu},
$$

where $T^{\mu \nu}$ is the flat energy-momentum pseudotensor of matter plus gravity, which interacts with the background radiation field. It satisfies the conservation law, $\partial_{\mu} T^{\mu \nu}(x)=0$, due to the Ward identities for graviton amplitudes.

The multipole expansion is then generated by taking the long wavelength limit, $|\vec{k}| \rightarrow 0$, or in position space by expanding the radiation field as follows:

$$
\widetilde{h}_{\mu \nu}(x)=\sum_{n=0}^{\infty} \frac{1}{n !} \vec{x}^{i_{1}} \cdots \vec{x}^{i_{n}} \partial_{i_{1}} \cdots \partial_{i_{n}} \widetilde{h}_{\mu \nu}\left(x^{0}, \overrightarrow{0}\right),
$$

where the point $\vec{x}=\overrightarrow{0}$ is taken to be the center of the composite object. This expansion is truncated at any finite $\mathrm{PN}$ order, as we have that $\vec{x} \cdot \vec{\nabla} \sim r / \lambda \sim v$. Substituting this expansion into equation (5.8), and decomposing the moments, $\int d^{3} x T^{\mu \nu} \vec{x}^{i_{1}} \cdots \vec{x}^{i_{n}}$, into irreducible representations of the rotation group, yields the classical multipole moments, which appear in equation (5.2). For example, at LO we have the total energy of the isolated binary system, $M=\int d^{3} x T^{00}$, which is the mass monopole term in equation (5.2); at the next order in the expansion we find the total angular momentum, $L^{i j}=$ $-\int d^{3} x\left(T^{0 i} x^{j}-T^{0 j} x^{i}\right)$. At the next order in the velocity, $v$, we then find for the mass (or 'electric') quadrupole

$$
I^{i j}=\left[\int d^{3} x\left(T^{00}+T^{k k}\right) x^{i} x^{j}\right]_{T F},
$$

where $\mathrm{TF}$ denotes the traceless part of the tensor.

The energy-momentum pseudotensor, $T^{\mu \nu}$, which is essentially the off-shell emission amplitude, should thus be computed in terms of the orbital DOFs. It is computed as a sum of Feynman graphs with internal potential gravitons and a single external (off-shell) 


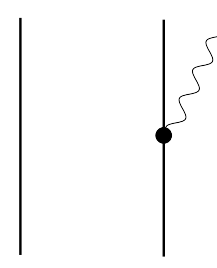

(a)

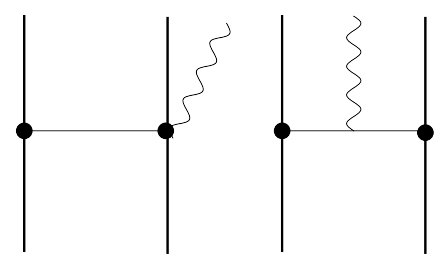

(c)

Figure 15. The Feynman diagrams that contribute up to the 1PN order of the mass quadrupole, $I^{i j}$ $[39,86]$. Unlike the conservative sector of the two-particle EFT, which contains graphs with internal potential gravitons only, and yields interaction potentials, as in figures 7-10 (and in the topologies of figures 5, 6, and 11-13), in the radiative sector we consider diagrams with external radiation modes. The latter are represented here by wiggly lines, whereas hereafter, all orbital modes are represented by solid lines. Contributing to the leading orders of the energy-momentum pseudotensor amplitude are the following diagrams: (a) Contribution to the leading $T^{\mu \nu}$ and subleading $T^{00}$ component; (b) Contribution to the subleading $T^{00}$ component; (c) Contribution to the subleading $T^{00}$ and leading $T^{i j}$ components. Diagram (c), which contains cubic self-interaction, is omitted when using the KK parametrization [88], similar to the 1PN order correction in the conservative sector; see figure 7 .

radiation graviton, so that the potential modes are integrated out as in equation (2.12) and in section 4.2. Figure 15 shows the graphs that contribute up to the $1 \mathrm{PN}$ order of the mass quadrupole, $I_{i j}$. To compute these diagrams one needs to have the Feynman rules of the propagators of the potential modes as in section 4.2 , but also of the worldline couplings and self-interaction vertices, which contain modes of the two types, potential and radiation, such as $m H \widetilde{h}$ on the worldline, or the cubic bulk vertex $\widetilde{h} H^{2}$. The latter would be extracted from the purely gravitational action with the harmonic gauge fixing, using the background field method [39].

After this matching of the multipole moments is done, one can proceed to compute the PN corrections to the radiation observables, such as the GW energy flux (or emitted power) or GW amplitude. In [86] it was demonstrated how to undertake this matching of the multipole moments at the $1 \mathrm{PN}$ order as shown in figure 15, and subsequently the 1PN order correction for the emitted power was reproduced. In [87] a general multipole expansion of the action linear in the radiation field specified the mass and current multipole moments to all orders in terms of the energy-momentum pseudotensor, and consequently the radiated power and the radiation amplitude. In $[88,89]$ the $1 \mathrm{PN}$ order correction to all mass multipoles was explicitly derived. In addition, multipole moments at NLO, dependent on the spins of the compact constituents, were tackled in [90, 91].

The linear coupling of radiation gravitons to the multipole moments, e.g. to the mass quadrupole, as shown in figure 16(a), has non-linear corrections due to gravitational selfinteractions, as shown in figures 16, 17, commonly referred to as hereditary effects. Not only are these relevant PN corrections, but they also involve logarithmic singularities, related with classical RG flows of the multipole moments from the orbital to the radiation scales [86]. We recall that the relevant expansion parameter of the EFT of a composite particle is $r / \lambda \sim v$, which just amounts to a multipole expansion, where the radiation sourced by the $l$-pole moment is suppressed by a relative factor of $v^{l}$. However, tail effects at the radiation 


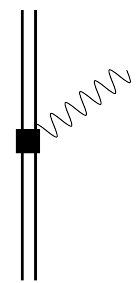

(a)

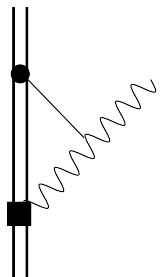

(b)

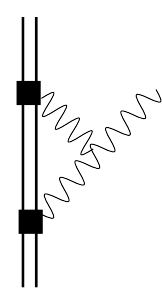

(c)

Figure 16. Radiation and hereditary effects in the EFT of the composite particle. Here the black square boxes and the sphere represent the time-dependent mass quadrupole, $I_{i j}$, and total mass monopole, $M$, moments of the binary, respectively: (a) The diagrams in figure 15 match onto this diagram in the effective theory of the composite particle, which depicts the quadrupole radiation [39]; (b) The leading tail effect, which enters at the relative 1.5PN order. The subleading corrections at the relative $3 \mathrm{PN}$ order, as will be shown in figure 17, were also reproduced in [39, 86]; (c) The leading memory effect, which enters at the relative $2.5 \mathrm{PN}$ order, related with the $\mathrm{LO}$ radiation reaction; see figures $18(\mathrm{~b} 2), 20(\mathrm{a})$ in the following $[92,99]$.

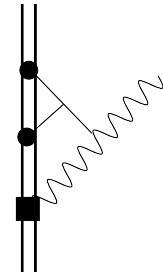

(a)

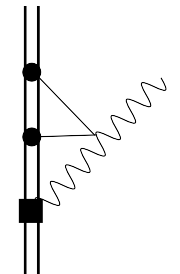

(b)

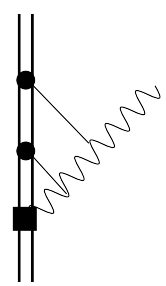

(c)

Figure 17. Radiation and subleading tail effects in the EFT of the composite particle. The NLO corrections to the tail effect in figure 16(b), which enter at the relative 3PN order, are reproduced in $[39,86]$. All of the graphs are logarithmically UV divergent, related with the classical RG flow of the mass quadrupole moment, $I_{i j}$, from the orbital to the radiation scales [86].

scale, which arise from non-linear interaction with the gravitational potential binding the composite particle, scale as $G M / \lambda \sim v^{3}$. Hence, the leading non-linear correction in figure $16(\mathrm{~b})$, which shows the scattering of outgoing radiation gravitons from potential gravitons sourced by the total mass of the system, enters at the relative $1.5 \mathrm{PN}$ order.

Long distance infrared (IR) logarithmic singularities already appear at the leading nonlinear order, but they are eventually seen to explicitly cancel out from physical observables [86]. However, the subleading non-linear corrections to the tail effect in figure 16(b), which are shown in figure 17, also involve logarithmic short distance UV singularities. These corrections enter at the relative $3 \mathrm{PN}$ order and are dubbed the 'tail of tail' [86]. The UV singularities concerned represent a real UV divergence of the EFT of the composite particle, and involve renormalization of the theory. All these UV divergences can be rendered finite by a renormalization of the multipole moments. The renormalized moments exhibit scale dependence described by a renormalization group ( $R G$ ) equation. This RG equation generates the entire series in powers of $v^{3}$ of leading UV logarithms. This set of terms is generic, that is, independent of the short distance physics of the composite particle. It 
is a prediction of the $\mathrm{RG}$ equation that this series of terms appears in long wavelength gravitational radiation from any compact binary system.

These subleading tail corrections and logarithms were reproduced and further understood via the EFT approach in [86], and amount to a two-loop computation, where graphs (a) and (b) of figure 17 in fact easily reduce to nested one-loop computations. A scale dependence for a renormalized moment was then introduced, in order to compensate for the explicit scale dependence of the logarithm, through the following RG equation for the mass quadrupole moment:

$$
\mu \frac{d}{d \mu} I_{i j}(\omega, \mu)=-\frac{214}{105}(G M \omega)^{2} I_{i j}(\omega, \mu)
$$

This equation then has the following solution:

$$
I_{i j}(\omega, \mu)=\left(\mu / \mu_{0}\right)^{-\frac{214}{105}\left(G M_{0} \omega\right)^{2}} I_{i j}\left(\omega, \mu_{0}\right),
$$

where the scale $\mu_{0}$ is taken as the characteristic UV scale of the composite object size, that is, $\mu_{0} \sim 1 / r$, where in the $\mathrm{PN}$ regime it holds that $\mu / \mu_{0} \sim v$. Thus we have a running of the theory of the composite particle between the scales $r$ and $r / v$, and a classical RG flow of physical observables, where the RG analysis is universal, and applies to gravitational radiation from any localized composite particle. The RG flow is shown here for the quadrupole moment but would apply similarly to further multipole moments. However, let us suspend the analysis of the subleading RG flow of the total mass of the composite particle $[92,102]$, in order to introduce an essential formal progression for this stage.

As we noted the radiating system is dissipative, as there is no incoming radiation at the asymptotic past, and one should proceed to impose boundary conditions, which are not symmetric in time, in order to obtain real time observables such as the gravitational waveform or radiation reaction effects. Using the effective action of the composite particle presented in equations (5.1) and (5.2), and the consequent standard Feynman propagators, which account for a pure in-(out-)going wave at past (future) infinity, would lead to a noncausal evolution. The related in-out generating functional, $Z$, as in equation (5.3), which is useful for the calculation of scattering amplitudes, yields matrix elements rather than the real time expectation values. Further, the in-out effective action, $\Gamma$, as in equation (5.5), generates EOMs for the worldline DOFs, which are in general neither real nor causal as they should be [94, 99, 103]. In order to consistently implement retarded boundary conditions, and proceed to use retarded propagators when integrating out the radiation field, we need to construct an action in which time reversal is broken. In fact, there exists a consistent framework in QFT to define such an action to describe non-conservative evolution, known as the closed time path (CTP; or the 'in-in', or Schwinger-Keldysh) formalism [150, 151], commonly used in non-equilibrium QFT [152].

In the CTP formalism the time contour in the path integral formulation goes from the asymptotic past hypersurface to a constant hypersurface at some possibly finite time, and back to the asymptotic past on another time branch. Therefore in the CTP action the actions of two different histories of the DOFs are compared by computing their difference, where in the first history there is forward time evolution, and the second history evolves 
backward through time from its final to initial configuration. It is important to stress that the values of the DOFs at the final time are set to be equal, which is referred to as the 'equality condition' (or the 'CTP boundary condition'), but their value is not fixed. Hence, the CTP formulation is an initial value formulation, as opposed to the in-out formulation, which is a boundary value formulation, where the paths go from the asymptotic past to the asymptotic future. However, in both formulations there is only one relevant physical history, so the two histories in the CTP formulation are both set equal to the physical one, after taking the variations of the CTP action, which is referred to as the 'physical limit'.

Let us review the CTP formalism to gain a more concrete understanding of how it works, where here we consider its quantum formulation, and assume that it is eventually applied in the classical limit. As one would expect, the latter limit is found to be equivalent to various independent classical formulations, which were made for non-conservative evolution $[88,103,104]$, yet here we want to avoid restricting the discussion to the specific classical context, but rather to keep it on a more generic fundamental level. Given a general action for a scalar field theory, $S[\phi]$, the DOFs are doubled, and the following action is defined as follows:

$$
S_{\mathrm{CTP}}\left[\phi_{1}, \phi_{2}\right] \equiv S\left[\phi_{1}\right]-S\left[\phi_{2}\right]^{*} .
$$

The CTP generating functional of correlation functions of such a scalar field theory, coupled to a source $J_{1}$ during the forward evolution in time and a source $J_{2}$ for the evolution backward in time, is defined by the following path integral:

$$
\begin{aligned}
Z_{\mathrm{CTP}}\left[J_{1}, J_{2}\right] & \equiv \exp ^{i W_{\mathrm{CTP}}\left[J_{1}, J_{2}\right]} \\
& \equiv \int \mathcal{D} \phi_{1} \mathcal{D} \phi_{2} \exp \left[i S_{\mathrm{CTP}}\left[\phi_{1}, \phi_{2}\right]+i \int d^{4} x\left(J_{1} \phi_{1}-J_{2} \phi_{2}\right)\right],
\end{aligned}
$$

with the equality condition, $\lim _{t \rightarrow t_{f}} \phi_{1}(x)-\phi_{2}(x)=0$, at some final time, $t_{f}$.

Two mean fields are considered to be derived from the following variations:

$$
+\frac{\delta}{\delta J_{1}(x)} W_{\mathrm{CTP}}=\left\langle\phi_{1}(x)\right\rangle, \quad-\frac{\delta}{\delta J_{2}(x)} W_{\mathrm{CTP}}=\left\langle\phi_{2}(x)\right\rangle,
$$

after which one sets $J_{1}=J_{2}=0$, and then $\left\langle\phi_{1}\right\rangle=\left\langle\phi_{2}\right\rangle \equiv \phi_{\text {mean }}$, which amounts to the physical constraint, that $\phi_{1}=\phi_{2}$ on-shell. We can also introduce the CTP effective action as the Legendre transform of $W_{\mathrm{CTP}}$ :

$$
\Gamma_{\mathrm{CTP}}\left[\left\langle\phi_{1}\right\rangle,\left\langle\phi_{2}\right\rangle\right]=W_{\mathrm{CTP}}\left[J_{1}, J_{2}\right]-\int d^{4} x\left[J_{1}\left\langle\phi_{1}\right\rangle-J_{2}\left\langle\phi_{2}\right\rangle\right]
$$

and for the mean fields we have the equations

$$
\frac{\delta \Gamma_{\mathrm{CTP}}}{\delta\left\langle\phi_{1}\right\rangle}=-J_{1}, \quad \frac{\delta \Gamma_{\mathrm{CTP}}}{\delta\left\langle\phi_{2}\right\rangle}=+J_{2}
$$

where we set $J_{1}=J_{2}=0$ to obtain the EOMs for the physical mean field, $\phi_{\text {mean }}$, and the two equations turn out to be equivalent. 
The propagators between $\phi_{A}$ and $\phi_{B}$, where $A, B=1,2$ denote the time branch within the time contour, are ordered in the following matrix:

$$
G_{A B}=\left(\begin{array}{cc}
G_{F} & -G_{-} \\
-G_{+} & {\left[G_{F}\right]^{*}}
\end{array}\right),
$$

where $G_{F}$ is the Feynman propagator given by

$$
G_{F}\left(x, x^{\prime}\right)=\theta\left(t-t^{\prime}\right) \Delta_{+}\left(x, x^{\prime}\right)+\theta\left(t^{\prime}-t\right) \Delta_{-}\left(x, x^{\prime}\right),
$$

and the propagators $G_{ \pm} \equiv \Delta_{ \pm}$read as follows:

$$
\Delta_{ \pm}\left(x, x^{\prime}\right)=\int \frac{d^{3} \vec{k}}{(2 \pi)^{3}} e^{ \pm i k_{0}\left(t-t^{\prime}\right)} \frac{e^{-i \vec{k} \cdot\left(\vec{x}-\vec{x}^{\prime}\right)}}{2 k_{0}} .
$$

So $G_{A B}$ in equation (5.18) stands for the 'path-ordered' propagators: Path-ordering is equivalent to time-ordering for points on the first time branch, anti-time-ordering on the second time branch, and placing points on the second branch to the left of points on the first branch.

It is useful to change the basis to the Keldysh representation by rewriting the sources and fields in terms of their average and the relative difference between the two time branches, namely $J_{+} \equiv\left(J_{1}+J_{2}\right) / 2, J_{-} \equiv J_{1}-J_{2}$, and $\phi_{+} \equiv\left(\phi_{1}+\phi_{2}\right) / 2, \phi_{-} \equiv \phi_{1}-\phi_{2}$. The ' + ' field variable evolves forward in time, and satisfies the initial conditions, whereas the '-' field variable evolves backward in time with the equality condition at the final time. When taking the physical limit, $\phi_{+} \rightarrow \phi$, i.e. the average of the two histories is identified with the physical one, while their difference vanishes, $\phi_{-} \rightarrow 0$. The propagator matrix in the Keldysh basis, where now $A, B$ take the,+- labels, is then given by

$$
G_{A B}=\left(\begin{array}{cc}
0 & -i G_{\mathrm{adv}} \\
-i G_{\mathrm{ret}} & \frac{1}{2} G_{H}
\end{array}\right)
$$

where the retarded and advanced propagators are given by

$$
\begin{aligned}
-i G_{\mathrm{ret}}\left(x, x^{\prime}\right) & =\theta\left(t-t^{\prime}\right)\left(\Delta_{+}\left(x, x^{\prime}\right)-\Delta_{-}\left(x, x^{\prime}\right)\right), \\
G_{\mathrm{adv}}\left(x, x^{\prime}\right) & =G_{\mathrm{ret}}\left(x^{\prime}, x\right),
\end{aligned}
$$

and the diagonal entry in the matrix in equation (5.21) can in fact be disregarded, i.e. taken as 0 , in the classical limit.

The generalization of the above formulation from a scalar field theory to our gravitational effective theory of a composite particle is rather straightforward [99]. In our theory the generating functional, $Z_{\mathrm{CTP}}$, depends on current densities, $J^{\mu \nu}$ and $j_{\mu}$, which couple linearly to the radiation field, $\widetilde{h}_{\mu \nu}$, and the particle worldline coordinate, $x^{\mu}$, respectively, where both field and worldline DOFs are doubled. Variation of the corresponding generating functional, $W_{\mathrm{CTP}}$, with respect to the current density, $J^{\mu \nu}$, gives the one-point function of the radiation field. A partial Legendre transform of $W_{\mathrm{CTP}}$ with respect to $j_{\mu}$ gives the corresponding effective action functional, $\Gamma_{\mathrm{CTP}}$, whose variation yields the EOMs 
for the orbital motion of the compact objects. For the computation of Feynman diagrams within the CTP formalism, as in figures 18-20 below: CTP labels should also be included at each worldline-graviton coupling, with $n$ labels for a worldline coupling with $n$ gravitons, in order to keep track of the time branches in the CTP path integral, where a two-point function matrix similar to equation (5.21) should be used, and all CTP indices should be summed over.

As we noted classical formulations to handle dissipative systems were put forward, motivated by the radiating binary inspiral problem. Notably, a formulation, which extends Hamilton's principle to classical causal actions for generic non-conservative systems, was introduced in [103], and later extended for generic classical field theories in [104]. This provided an independent classical formulation, which was tested successfully against the CTP formalism of nonequilibrium QFT in the classical limit. Furthermore, a study of the classical origin of the quantum CTP formalism at the level of the action, which can also be generally applied at the level of the EOMs, was carried out independently in $[88,89]$. This study was also extended for a general spacetime dimension in $[105,106]$.

Let us resume the discussion on the renormalization of the total mass of the composite particle. The classical RG flow induces time dependence, and thus the time evolution of the renormalized mass can be studied by computing the expectation value of the energymomentum pseudotensor, using its conservation from the Ward identities and the CTP formalism adapted to the classical system [92]. The RG flow of the total mass can also be inferred from an analysis of the radiation reaction as in [102], using the purely classical dissipative formulation devised in $[103,104]$; see figure 20 and the related discussion below.

Let us outline here the analysis in terms of the stress-energy pseudotensor, following the work in [92]. First, the metric is expanded into a weak background, $\tilde{g}_{\mu \nu}=\eta_{\mu \nu}+\tilde{h}_{\mu \nu}$, and a fluctuating field, $\hat{h}_{\mu \nu}=\widetilde{g}_{\mu \nu}-\tilde{g}_{\mu \nu}$ (note that the weak background, $\tilde{g}$, is denoted with a tilde, whereas the total radiation metric, $\widetilde{g}$, is denoted with a wide tilde). Then, the fluctuations are integrated out according to the CTP formalism to obtain the effective action as follows:

$$
\exp ^{i \Gamma\left[\tilde{h}_{+}, \tilde{h}_{-}, M, I\right]}=\int \mathcal{D} \hat{h}_{+} \mathcal{D} \hat{h}_{-} \exp ^{i\left[S\left[\widetilde{g}_{+}, M, I\right]-S\left[\widetilde{g}_{-}, M, I\right]\right]}
$$

where the field DOFs have been doubled, and transformed into the Keldysh representation, and the CTP boundary conditions are imposed on the fluctuating modes. Then, the real time expectation value of the effective energy-momentum pseudotensor is given by

$$
\left\langle T_{\mu \nu}(x)\right\rangle=-\left.\frac{2}{\sqrt{\widetilde{g}_{+}}} \frac{\delta \Gamma}{\delta \tilde{g}_{+}^{\mu \nu}(x)}\right|_{\tilde{h}_{+}=\tilde{h}_{-}=0},
$$

where after the variation we set the external graviton, e.g. $\tilde{h}_{+}$, to 0 .

Hence, we find the expectation value $\left\langle T_{\mu \nu}\right\rangle$ by considering Feynman graphs with one (off-shell) external graviton, as can be seen in figures 18 and 19. The first graphs to consider, shown in figure 18, give the leading contribution to the expectation value, and yield the time evolution of the total mass, from which one recovers the following well-known 


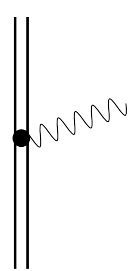

(a)

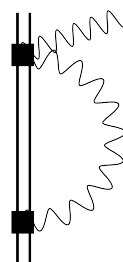

(b1)

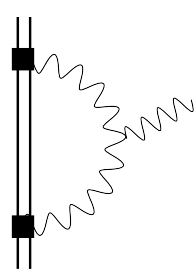

(b2)

Figure 18. The leading time dependence of the mass quadrupole and total mass can be found by considering the expectation value $\left\langle T^{\mu \nu}\right\rangle$ [92]. These diagrams are not logarithmically divergent, and they yield the well-known LO quadrupole radiation formula, which also corresponds to the LO radiation reaction, depicted in figure 20(a) [99].

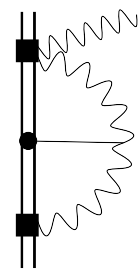

(a)

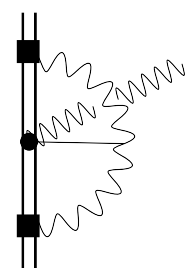

(c)

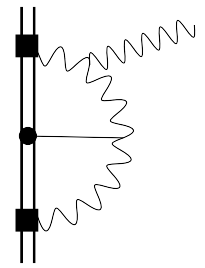

(b)

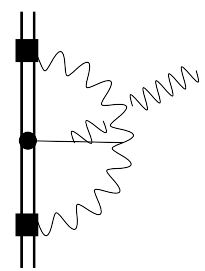

(d)

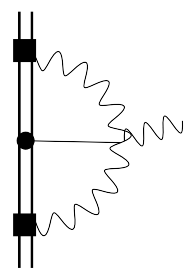

(e)

Figure 19. The classical RG flow of the composite mass can be found by considering the expectation value $\left\langle T^{\mu \nu}\right\rangle$ [92]. These diagrams are logarithmically UV divergent, and yield classical RG running of the mass. This RG flow of the mass can also be inferred from the leading non-linear radiation reaction, corresponding to graph (c) of figure 20 [102].

quadrupole radiation formula:

$$
\langle\dot{M}\rangle=-\frac{G}{5}\left\langle I_{i j}^{(3)} I_{i j}^{(3)}\right\rangle,
$$

which accounts for the energy loss of the system to gravitational radiation. This corresponds to the LO radiation reaction depicted in graph (a) of figure 20 [99].

The diagrams in figure 18 are not logarithmically divergent, yet the non-linear corrections in figure 19 yield logarithmic UV divergences, which give rise to the mass renormalization, providing a new time-dependent RG equation for the mass. By considering the singular pieces of the graphs in figure 19 as $d-4 \rightarrow 0$ the following RG equation for the composite mass, $M(t)$, is found:

$$
\mu \frac{d}{d \mu} \ln \bar{M}=-2 G^{2}\left\langle I_{i j}^{(3)} I_{i j}^{(3)}\right\rangle(\mu)
$$




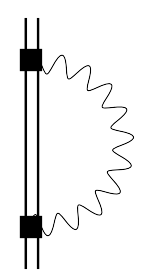

(a)

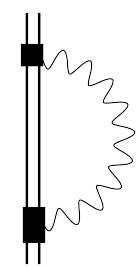

(b1)

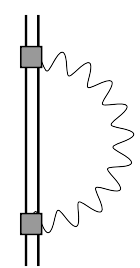

(b2)

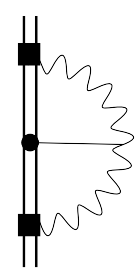

(c)

Figure 20. Radiation reaction in the effective theory of the composite particle for the PN binary inspiral, similar to the self-force in the effective point-particle theory for EMRIs [94]: (a) The LO effect at the 2.5PN order [39, 99]; (b1) and (b2) The NLO effect at the 3.5PN order [100]. Here the black rectangle represents the mass octupole, $I_{i j k}$, and the gray squares represent the current quadrupole, $J_{i j}$; (c) The leading non-linear effect, which enters at the $4 \mathrm{PN}$ order, tackled in $[101,102]$. As opposed to the former linear effects, this has a contribution to the conservative action.

where at this order $M(t)$ is simply replaced by its time average, $\bar{M}$. The mass is scale dependent due to the radiation backscattering off of the static background, as seen in figures 16(b), 17, 19, and 20(c). The RG running of the composite mass is suppressed with respect to that of the mass quadrupole, $I_{i j}$, so the coupled RG equations, equations (5.27) and (5.11), may be solved by using the solution in equation (5.12), assuming $\bar{M}$ is scale independent. The solution of the RG equation for the mass then reads as follows:

$$
\frac{\bar{M}(\mu)}{\bar{M}_{0}}=\exp \left[\frac{\left\langle I_{i j}^{(2)} I_{i j}^{(2)}\right\rangle\left(\mu_{0}\right)-\left\langle I_{i j}^{(2)} I_{i j}^{(2)}\right\rangle(\mu)}{\frac{214}{105} \bar{M}_{0}^{2}}\right],
$$

where the mass as defined in equation (5.2) is the energy of the conservative system, not including energy in gravitational radiation.

The solution to the coupled RG equations for $M$ and $I_{i j}$ provides the logarithms, which appear in the low frequency mode, $l=2$, distribution of emitted gravitons radiated from the binary. Thus the logarithms in the running composite mass and mass quadrupole can be predicted at higher PN orders. Similar to this analysis, which is derived from the conservation of the expectation value of the stress-energy pseudotensor, $\partial_{\mu}\left\langle T^{\mu \nu}(x)\right\rangle=0$, the evolution equation for other multipole moments, such as the total angular momentum of the system, can also be set up. An alternative analysis can be made by studying the radiation reaction, as in graph (c) of figure 20 for the $\mathrm{RG}$ flow of the composite mass, which was analyzed here using figure 19 .

The radiation reaction in the PN binary inspiral was tackled in an EFT approach in [99], where it is computed by integrating out the radiation field modes from the effective theory of the composite particle. In this work the LO effect at the $2.5 \mathrm{PN}$ order was reproduced (see also in [39]), as shown in graph (a) of figure 20. Next, the NLO effect at the 3.5PN order, which is still linear in $G$ as can be seen in graphs (b1) and (b2) of figure 20 , was reproduced in $[100]^{12}$. The leading non-linear tail effect, which yields a radiation

\footnotetext{
${ }^{12}$ Related with this progress, the LO finite size correction to the radiation reaction in classical electrodynamics was also found in [153]; see also [154, 155].
} 
reaction force at the 4PN order, was first approached in [101], and further studied in [102], using the abovementioned classical formulation for dissipative systems from [103, 104]. This non-linear effect, depicted in figure 20(c), contributes a piece, which is non-local in time, to the effective action of the composite particle in the point-mass sector [140], and it contributes to the binding energy logarithmic corrections, which were first obtained via the EFT approach in [92 ${ }^{13}$. Hence, unlike the linear effects of the radiation reaction in figure 20, the logarithmically divergent part at order $G^{2}$ from graph (c) is a conservative effect, which affects the orbital dynamics of the binary.

Graph (c) of figure 20 is a self-energy graph of the theory, i.e. a renormalization graph of the mass monopole of the effective theory of the composite particle, and thus it enables one to infer the RG flow of the composite mass [102]. In fact, the study in [102] indicates that the renormalization of the effective theory of the composite particle can be more efficiently approached via an analysis of the radiation reaction, rather than via an analysis, which involves the expectation value of the stress-energy pseudotensor as in [92]. Moreover, the analysis in [102] in terms of the radiation reaction reveals the subtle interplay between the radiation (or far) and the potential (or near) zones, where the counterterm for the UV divergence of the radiation reaction potential originates from an IR singularity in the orbital region.

Finally, the leading radiation reaction linear in the spins of the binary components, which enters at the 4PN order, was reproduced in [107], where the LO spin-orbit tail effect was also previously reproduced in [91]. The leading radiation reaction quadratic in the spins, including the backreaction from spin-induced finite size effects, which enters at the 4.5PN order, was computed in [108].

\subsection{EFTofPNG public code}

Eventually, one would like to capitalize on the outcome of the EFT framework for the PN binary inspiral to arrive at the practical inclusion of the various results in the GW flux and in the gravitational waveform amplitude and phase. This was indeed a significant driver in creating 'EFTofPNG' - a public Mathematica package, which incorporates the EFT framework for high precision Feynman computation in PN gravity [112]. In its current first version, it has applied to the conservative sector and covers the current state of the art in the field. The code was designed to be self-contained and modular with independent units, including a pipeline unit, in order to serve diverse purposes within the broad community, for which it was made accessible. An outline of the EFTofPNG package version 1.0 is depicted in figure 21. This is the first comprehensive code in PN theory made public, so as to fill in the current analytic gap for the major part of the gravitational waveform models, and complement current GW public codes, which include numerical codes, codes for BH perturbation theory [113], and codes for data analysis ${ }^{14}$. Moreover, it is expected

\footnotetext{
${ }^{13}$ The analogy of the leading non-linear tail effect to the well-known Lamb shift and Bethe logarithm from electrodynamics was also discussed in [156].

${ }^{14}$ We note a recent public numerical code, 'PRECESSION', which specifically studies the dynamics of spinning BHs in the PN regime [157]. However, this code deals with the outcome of a given PN dynamics, rather than deriving the PN theory like the EFTofPNG code does.
} 


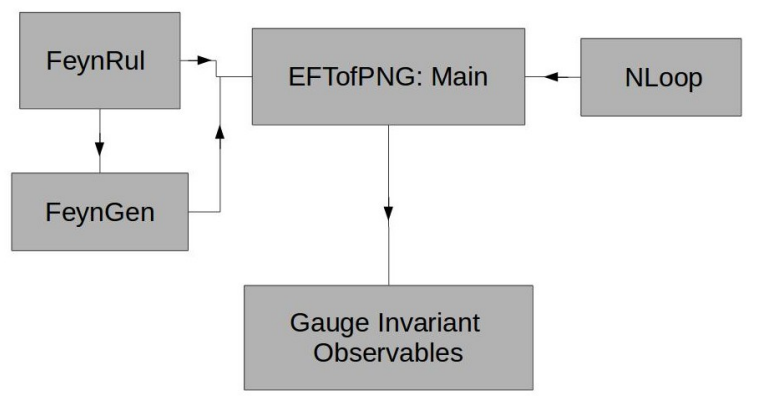

Figure 21. A sketch of the comprehensive 'EFTofPNG' public package version 1.0, which incorporates the EFT framework for high precision Feynman computation in PN gravity, including spin effects [112]. The code is modular with independent units, and a pipeline unit, where the flow among these units is shown here. This is the first public code in PN theory, and also serves as a generic high precision Feynman computation tool.

that the code will be continually extended and publicly developed to cover higher-order PN sectors, non-conservative sectors, and further GW observables. However, it should be stressed that beyond its obvious utility for PN gravity, the EFTofPNG is also a useful high precision Feynman computation tool, which handles the generic perturbative diagrammatic expansion in a unique and efficient way and contains a stand-alone multi-loop computation unit.

As for GW observables from the conservative sector, which are included in the code, we note that finally, neither the effective interaction potentials, nor the Hamiltonians, are gauge invariant (GI). However, the Hamiltonians are related to the total energy of the binary system, which is a global GI quantity, like the total angular momentum of the binary. In addition, for the simplified case of binaries with non-spinning components in quasi-circular orbits the energy depends only on the magnitude of the orbital angular momentum. This relation between binding energy and angular momentum is GI, and is thus a useful tool to evaluate different analytic and numerical descriptions of the binary dynamics [158]. There is another useful GI relation between the binding energy and the orbital frequency, that together with the energy flux of the GWs, can be used to derive the GW phasing. All these GI quantities and relations are also included in the EFTofPNG code. Finally, a further important useful GI quantity, to be possibly considered in the code in the future, is the periastron advance [159-161].

\section{$6 \quad$ Field theory for gravity at all scales}

With significant developments over the last decade, since its initiation, the field of EFTs in PN gravity has firmly demonstrated that seemingly disparate physical domains, such as QFT and classical gravity, are in fact related. This attests to the unity of physics. The EFT framework for PN gravity has proved to supply a robust methodology to boost the progress in PN theory, in particular for the timely study of GWs from the inspiral of comparable mass compact binaries. GW observations are thereby expected to carry insightful implications the other way around: For various subjects in theoretical physics, 
from QCD and gravity, to astrophysics and cosmology. As was highlighted in section 1, PN theory exclusively covers a considerable portion of the CBC GW signal, which must be accurately modeled in order to extract the utmost possible information from GW data. Numerical simulations cannot in essence cover the NR regime, and all the more so when the spins of the components of the binary are taken into account and their capacity becomes extremely limited.

Concerning the output of the EFT methodology for the binary inspiral in the conservative sector, there are two key ingredients of the orbital dynamics, which should be produced in a straightforward manner in order to improve the accuracy of theoretical GW templates: The EOMs and the Hamiltonians. This was attained in the EFT framework, in particular in the realistic yet much more challenging case where spins are present [75], and is incorporated in the pipeline unit of the EFTofPNG code [112]. It should be noted that standard Hamiltonians still need to be further worked out into EOB Hamiltonians in order to be incorporated into the EOB GW models. This is currently not implemented up to the level of PN accuracy attained in the spin sectors. Further, more generally, due to the complexity of spin effects, their incorporation into GW templates falls far behind the state-of-the-art results of PN theory. As for the EOMs of the components of the binary, progress should also be made in finding solutions to the EOMs to high PN order, a challenging task which again lags behind the state-of-the-art PN theory and even more so in the spin sectors. Interestingly, in [162], a dynamical RG method, designed to calculate such closed form solutions to a desirable accuracy without orbit averaging, was put forward.

Despite the notable progress within the EFT approach to the binary inspiral, there is still much room and demand for development in the field. First, in general, our conceptual grasp of classical spins in gravity should be further improved. In particular, the EFT framework via coset construction in [77], suitable for slowly rotating gravitating objects, should be scrutinized, which may possibly lead to a unified formulation suitable for both slowly and rapidly rotating objects in the appropriate limits. In any case, building on the formulation in [77], concrete PN predictions for slowly rotating objects should be produced. Spin-induced Wilson coefficients, which play a role at least as of the $2 \mathrm{PN}$ order, should be studied via a formal matching procedure. Further, the treatment of the nonconservative sectors within the EFT approach seems to be underdeveloped with respect to other methods in PN theory, and with respect to the progress accomplished within the EFT approach in the conservative sector. In particular, it should be stressed that tail and dissipative effects manifest in the late inspiral phase, which constitutes the transition into the strong field regime. Finally, public codes like the EFTofPNG package should be further developed in order to improve in computational efficiency, but more importantly, they should be extended along with formal advancement in the field, especially so as to cover the non-conservative sectors.

Whether one is strictly interested in to analytically covering the GW CBC signal, entering the strong field regime as well, or much more generally looking to obtain a complete theory of gravity at all scales, it is clear that field theory advances at all levels should be invoked. First, standard working knowledge in scattering amplitudes can be imported from gauge theory to gravity [137], and vice versa, to further advance high precision com- 
putation, as was demonstrated in [55, 129]. Furthermore, modern advances in scattering amplitudes, such as the BCFW on-shell recursion relations [163] and unitarity methods, were demonstrated to reproduce low order results in classical gravity $[164,165]$. These advances imply that tree level data encode all multiplicity at the integrand level, i.e. that the integrands of extremely complicated multi-loop predictions can be verified systematically and invariantly with compact on-shell tree level data. Thus, more generally, it has been shown how to extract classical higher-order loop results for gravitational scattering from advanced on-shell unitary methods and for higher spin particles in [166-170]. In order to actually go into the strong field regime of the CBC signal, the gravitational scattering of two BHs, which can be deduced from the quantum gravitational scattering amplitude of two particles, was mapped into the EOB framework [171-174]. It was indeed recently discussed how to strategically get from scattering amplitudes, using generalized unitarity, to the classical scattering of two BHs $[175,176]$.

Proceeding to candidate theories of quantum gravity, i.e. extending gravity in the $\mathrm{UV}$, intriguing and powerful duality and correspondence relations have been discovered in the context of scattering amplitudes, between supersymmetry and supergravity theories [177-180]. The color-kinematics, or so-called BCJ duality, and the related double copy correspondence, state that Yang-Mills amplitudes, for example, can be mapped onto their gravity theory counterparts by applying a prescribed set of color to kinematics replacement rules [181]. First, this correspondence provides an exciting opportunity to reveal more on the underlying origin of these relations in the fundamental structure of these QFTs, gauge and gravity theories, and thus extend our understanding and reach of both. However, this correspondence also offers a compelling novel approach for handling perturbative (and exact) computation within classical gravity, and particularly for studying GWs. In fact, the exploration of how to formulate the double copy correspondence in the classical perturbative context has been already initiated in a recent series of works [182-194].

Turning instead to very large scales, we are once again puzzled by the observational discovery of the accelerated expansion of the Universe, or the threefold equivalence of the IR structure of gauge and gravity theories, among soft theorems, memory effects, and asymptotic symmetry. These have led us to the realization that our understanding of gravity is not only lacking at the quantum regime, but also at the classical IR one. In fact, the problems of dealing with gravity in the UV and IR sides may well be tied together. Let us then discuss first the triple classical and quantum equivalence noted above. Soft theorems are statements about the universal characteristic behavior of scattering amplitudes at low energies, and they tell us about the IR structure of gauge and gravity theories with massless particles (see, e.g. [195]). In particular, the equivalence between soft graviton theorems and gravitational memory effects was recently demonstrated in [196, 197]. The former concerns momentum space poles in scattering amplitudes, while the latter concerns a shift in asymptotic data between late and early times, and they are simply connected via a Fourier transform. Furthermore, there is going development on gravitational memory effects and their observable imprint in GWs [198, 199], their manifestation in cosmological GWs [200, 201], and related further advancements in soft theorems [202, 203].

However, the cosmological constant problem and dark matter puzzle call for alternative 
theories of gravity at large scales, even if only for the classical regime. Indeed, a plethora of alternative theories of gravity in the IR has been put forward through the years: Theories with extra fields, such as massive gravity (see, e.g. [204, 205]) or Horndeski theories (see, e.g. [206]); higher derivative and non-local theories, e.g. Galileons (see, e.g. [206]); or higherdimensional theories, in the context of large extra dimensions in particle physics, or inspired by string theory. We also refer the reader to the comprehensive reviews in [207, 208]. The theoretical and phenomenological study of these alternative theories serves to develop general tests of gravity, also based on GR, through the extraction of the latest information from GW data (see, e.g. [209]). Conversely, the ongoing advances in GW and cosmological observations may greatly benefit our ability to pin down an ultimate viable theory of gravity (see, e.g. [210, 211]).

Different aspects of such alternative theories have been already studied by applying the EFT approach (see, e.g. [212, 213]). Preliminary explicit implementations in radiating binary systems in Galileon theories have been made in [214], and also following the EFT framework from $[39,41]$ in $[215,216]$, and were recently followed up with numerical work in [217]. Thus, it is desirable to apply the EFT framework, replacing GR with other alternative theories of gravity, to produce predictions for the possible GW signal from binaries. Furthermore, building on the $n$-body case initially explored with the EFT approach in [52], it may be beneficial for the study of such alternative theories to model GWs from $n$-body systems, which may also be facilitated using higher multiplicities from scattering amplitudes. Although two-body systems are much more abundant in nature, if GWs were to be detected, e.g. from three-body systems, this would provide for richer testing of GR, since the two-body sector is a degenerate case as far as the non-linearities of GR are concerned. At the practical level the EFTofPNG code, currently implemented for binaries in GR, can be easily accommodated to handle the more generic case in such modified theories of gravity.

It is noteworthy that the coupling of spin to the various fields in each candidate theory of gravity, classical or quantum, plays a crucial role in distinguishing among these theories. This further highlights the vital importance of gaining a profound understanding of spin, in particular also in classical gravity.

To conclude, there are myriad prospects of using modern insights and tools from the realm of field theory to study gravity, which will surely be taken advantage of in the endeavor to zero in on a complete theory of gravity across all scales. Indeed, also in this broader sense, this line of research, which has been advanced with the use of EFTs in PN gravity, is certain to continue developing in the years to come and lead us towards resolving at least some of the formidable fundamental puzzles that we currently face.

\section{Acknowledgments}

I am grateful to John Joseph Carrasco for his significant encouragement and support, and for comments on the manuscript. I would like to thank Alessandra Buonanno, Yi-Zen Chu, Thibault Damour, Stefano Foffa, Walter Goldberger, Pierpaolo Mastrolia, Riccardo Sturani, Christian Sturm, and Pierre Vanhove for their comments on the manuscript and 
support, and to Barak Kol and Ira Rothstein for support. I would like to warmly acknowledge my collaborators on this line of research, Barak Kol, Michael Smolkin, and Jan Steinhoff. Finally, I kindly thank Alexandre Le Tiec for allowing to use the plot in figure 1 in this review.

It is with great pleasure that I extend my gratitude to Feng-Li Lin and the Taipei GW group, in particular to Chian-Shu Chen, and Sheng-Lan Ko for the kind and warm hospitality, and for their keen interest in understanding the subtleties of the topic throughout my lecture series.

The work of ML is supported by the European Research Council under the European Union's Horizon 2020 Framework Programme FP8/2014-2020 "preQFT" grant no. 639729, "Strategic Predictions for Quantum Field Theories" project.

\section{References}

[1] R. A. Hulse and J. H. Taylor, Discovery of a pulsar in a binary system, Astrophys. J. 195 (1975) L51-L53.

[2] J. H. Taylor and J. M. Weisberg, A new test of general relativity: Gravitational radiation and the binary pulsar PSR 1913+16, Astrophys. J. 253 (1982) 908-920.

[3] J. M. Weisberg and J. H. Taylor, Observations of Post-Newtonian Timing Effects in the Binary Pulsar PSR 1913+16, Phys. Rev. Lett. 52 (1984) 1348-1350.

[4] T. Damour and N. Deruelle, General relativistic celestial mechanics of binary systems. II. The post-newtonian timing formula, Ann. Inst. Henri Poincaré Phys. Théor. 44 (1986), no. $3263-292$.

[5] "LIGO webpage." http://www.ligo.caltech.edu.

[6] "GEO600 webpage." http://www.geo600.org.

[7] "Virgo webpage." http://www.virgo-gw.eu.

[8] LIGO, VIRGO Collaboration, B. P. a. o. Abbott, Observation of Gravitational Waves from a Binary Black Hole Merger, Phys. Rev. Lett. 116 (2016) 061102, [arXiv: 1602.03837].

[9] Virgo, LIGO Scientific Collaboration, B. P. a. o. Abbott, GW151226: Observation of Gravitational Waves from a 22-Solar-Mass Binary Black Hole Coalescence, Phys. Rev. Lett. 116 (2016) 241103, [arXiv: 1606.04855].

[10] VIRGO, LIGO Scientific Collaboration, B. P. Abbott et al., GW170104: Observation of a 50-Solar-Mass Binary Black Hole Coalescence at Redshift 0.2, Phys. Rev. Lett. 118 (2017) 221101, [arXiv: 1706.01812].

[11] Virgo, LIGO Scientific Collaboration, B. P. Abbott et al., GW170608: Observation of a 19-solar-mass Binary Black Hole Coalescence, Astrophys. J. 851 (2017) L35, [arXiv: 1711.05578].

[12] Virgo, LIGO Scientific Collaboration, B. P. Abbott et al., GW170814: A Three-Detector Observation of Gravitational Waves from a Binary Black Hole Coalescence, Phys. Rev. Lett. 119 (2017) 141101, [arXiv:1709.09660].

[13] Virgo, LIGO Scientific Collaboration, B. Abbott et al., GW170817: Observation of 
Gravitational Waves from a Binary Neutron Star Inspiral, Phys. Rev. Lett. 119 (2017) 161101, [arXiv:1710.05832].

[14] LIGO Scientific, Virgo Collaboration, B. P. Abbott et al., Properties of the binary neutron star merger GW170817, Phys. Rev. X9 (2019) 011001, [arXiv:1805.11579].

[15] LIGO Scientific, Virgo Collaboration, B. P. Abbott et al., GW170817: Measurements of neutron star radii and equation of state, Phys. Rev. Lett. 121 (2018) 161101, [arXiv: 1805.11581].

[16] Virgo, LIGO Scientific Collaboration, B. P. a. o. Abbott, Tests of general relativity with GW150914, Phys. Rev. Lett. 116 (2016) 221101, [arXiv:1602.03841].

[17] Virgo, LIGO Scientific Collaboration, B. P. a. o. Abbott, Properties of the Binary Black Hole Merger GW150914, Phys. Rev. Lett. 116 (2016) 241102, [arXiv:1602.03840].

[18] "KAGRA webpage." http://gwcenter.icrr.u-tokyo.ac.jp/en.

[19] "IndiGO webpage." http://www.gw-indigo.org.

[20] KAGRA, LIGO Scientific, VIRGO Collaboration, B. P. Abbott et al., Prospects for Observing and Localizing Gravitational-Wave Transients with Advanced LIGO, Advanced Virgo and KAGRA, Living Rev. Rel. 21 (2018) 3, [arXiv:1304.0670].

[21] M. Punturo et al., The Einstein Telescope: A third-generation gravitational wave observatory, Class. Quant. Grav. 27 (2010) 194002.

[22] LIGO Scientific Collaboration, B. P. Abbott et al., Exploring the Sensitivity of Next Generation Gravitational Wave Detectors, Class. Quant. Grav. 34 (2017) 044001, [arXiv: 1607.08697].

[23] "ESA LISA webpage." http://sci.esa.int/lisa.

[24] Korean Gravitational Wave Group Collaboration, H. J. Paik, H. M. Lee, K. Cho, and J. Kim, Gravitational-wave Detectors and a New Low-frequency Detector SOGRO, New Phys. Sae Mulli 66 (2016), no. 3 272-282.

[25] S. Sato et al., The status of DECIGO, J. Phys. Conf. Ser. 840 (2017) 012010.

[26] TianQin Collaboration, J. Luo et al., TianQin: a space-borne gravitational wave detector, Class. Quant. Grav. 33 (2016) 035010, [arXiv:1512.02076].

[27] A. Buonanno and B. S. Sathyaprakash, Sources of Gravitational Waves: Theory and Observations, in A. Ashtekar, B.K. Berger, J. Isenberg and M. MacCallum, "General Relativity and Gravitation : A Centennial Perspective,", pp. 287-346. 2015. arXiv: 1410.7832.

[28] C. V. Vishveshwara, Scattering of Gravitational Radiation by a Schwarzschild Black-hole, Nature 227 (1970) 936-938.

[29] C. V. Vishveshwara, Stability of the schwarzschild metric, Phys. Rev. D1 (1970) 2870-2879.

[30] L. Blanchet, Gravitational Radiation from Post-Newtonian Sources and Inspiralling Compact Binaries, Living Rev. Rel. 17 (2014) 2, [arXiv:1310.1528].

[31] G. Schäfer and P. Jaranowski, Hamiltonian formulation of general relativity and post-Newtonian dynamics of compact binaries, Living Rev. Rel. 21 (2018) 7, [arXiv: 1805.07240].

[32] F. Pretorius, Evolution of binary black hole spacetimes, Phys. Rev. Lett. 95 (2005) 121101, 
[gr-qc/0507014].

[33] A. Le Tiec, The Overlap of Numerical Relativity, Perturbation Theory and Post-Newtonian Theory in the Binary Black Hole Problem, Int. J. Mod. Phys. D23 (2014) 1430022, [arXiv:1408.5505].

[34] A. Buonanno and T. Damour, Effective one-body approach to general relativistic two-body dynamics, Phys.Rev. D59 (1999) 084006, [gr-qc/9811091].

[35] A. Buonanno and T. Damour, Transition from inspiral to plunge in binary black hole coalescences, Phys. Rev. D62 (2000) 064015, [gr-qc/0001013].

[36] T. Damour, The General Relativistic Two Body Problem and the Effective One Body Formalism, Fundam. Theor. Phys. 177 (2014) 111-145, [arXiv:1212.3169].

[37] M. Hannam, P. Schmidt, A. Bohé, L. Haegel, S. Husa, F. Ohme, G. Pratten, and M. Pürrer, Simple Model of Complete Precessing Black-Hole-Binary Gravitational Waveforms, Phys. Rev. Lett. 113 (2014) 151101, [arXiv:1308.3271].

[38] S. Khan, S. Husa, M. Hannam, F. Ohme, M. Pürrer, X. Jiménez Forteza, and A. Bohé, Frequency-domain gravitational waves from nonprecessing black-hole binaries. II. A phenomenological model for the advanced detector era, Phys. Rev. D93 (2016) 044007, [arXiv: 1508.07253].

[39] W. D. Goldberger and I. Z. Rothstein, An Effective field theory of gravity for extended objects, Phys.Rev. D73 (2006) 104029, [hep-th/0409156].

[40] W. D. Goldberger and I. Z. Rothstein, Towers of Gravitational Theories, Gen. Rel. Grav. 38 (2006) 1537-1546, [hep-th/0605238]. [Int. J. Mod. Phys.D15,2293(2006)].

[41] W. D. Goldberger, Les Houches lectures on effective field theories and gravitational radiation, in Les Houches Summer School - Session 86: Particle Physics and Cosmology: The Fabric of Spacetime Les Houches, France, July 31-August 25, 2006, 2007. hep-ph/0701129.

[42] Y.-Z. Chu, W. D. Goldberger, and I. Z. Rothstein, Asymptotics of d-dimensional Kaluza-Klein black holes: Beyond the Newtonian approximation, JHEP 03 (2006) 013, [hep-th/0602016].

[43] B. Kol and M. Smolkin, Classical Effective Field Theory and Caged Black Holes, Phys. Rev. D77 (2008) 064033, [arXiv:0712.2822].

[44] R. Emparan, T. Harmark, V. Niarchos, and N. A. Obers, World-Volume Effective Theory for Higher-Dimensional Black Holes, Phys. Rev. Lett. 102 (2009) 191301, [arXiv:0902.0427].

[45] J. B. Gilmore, A. Ross, and M. Smolkin, Caged black hole thermodynamics: Charge, the extremal limit, and finite size effects, JHEP 09 (2009) 104, [arXiv:0908.3490].

[46] R. Emparan, T. Harmark, V. Niarchos, and N. A. Obers, Essentials of Blackfold Dynamics, JHEP 03 (2010) 063, [arXiv: 0910.1601].

[47] B. Kol, Classical Effective Field Theory for Weak Ultra Relativistic Scattering, JHEP 07 (2011) 062, [arXiv: 1103.5741].

[48] B. Kol and M. Smolkin, Non-Relativistic Gravitation: From Newton to Einstein and Back, Class.Quant.Grav. 25 (2008) 145011, [arXiv:0712.4116].

[49] B. Kol and M. Smolkin, Einstein's action and the harmonic gauge in terms of Newtonian 
fields, Phys.Rev. D85 (2012) 044029, [arXiv:1009.1876].

[50] B. Kol, M. Levi, and M. Smolkin, Comparing space+time decompositions in the post-Newtonian limit, Class.Quant.Grav. 28 (2011) 145021, [arXiv:1011.6024].

[51] J. B. Gilmore and A. Ross, Effective field theory calculation of second post-Newtonian binary dynamics, Phys.Rev. D78 (2008) 124021, [arXiv:0810.1328].

[52] Y.-Z. Chu, The n-body problem in General Relativity up to the second post-Newtonian order from perturbative field theory, Phys.Rev. D79 (2009) 044031, [arXiv:0812.0012].

[53] S. Foffa and R. Sturani, Effective field theory calculation of conservative binary dynamics at third post-Newtonian order, Phys.Rev. D84 (2011) 044031, [arXiv:1104.1122].

[54] S. Foffa and R. Sturani, Dynamics of the gravitational two-body problem at fourth post-Newtonian order and at quadratic order in the Newton constant, Phys.Rev. D87 (2013) 064011, [arXiv:1206.7087].

[55] S. Foffa, P. Mastrolia, R. Sturani, and C. Sturm, Effective field theory approach to the gravitational two-body dynamics, at fourth post-Newtonian order and quintic in the Newton constant, Phys. Rev. D95 (2017) 104009, [arXiv: 1612.00482].

[56] D. Bini, T. Damour, and G. Faye, Effective action approach to higher-order relativistic tidal interactions in binary systems and their effective one body description, Phys.Rev. D85 (2012) 124034, [arXiv:1202.3565].

[57] B. Kol and M. Smolkin, Black hole stereotyping: Induced gravito-static polarization, JHEP 02 (2012) 010, [arXiv:1110.3764].

[58] Virgo, LIGO Scientific Collaboration, B. P. a. o. Abbott, Improved analysis of GW150914 using a fully spin-precessing waveform Model, Phys. Rev. X6 (2016) 041014, [arXiv: 1606.01210].

[59] D. Gerosa, Surprises from the spins: astrophysics and relativity with detections of spinning black-hole mergers, J. Phys. Conf. Ser. 957 (2018) 012014, [arXiv:1711.10038].

[60] I. Harry and T. Hinderer, Observing and measuring the neutron-star equation-of-state in spinning binary neutron star systems, Class. Quant. Grav. 35 (2018) 145010, [arXiv:1801.09972].

[61] R. A. Porto, Post-Newtonian corrections to the motion of spinning bodies in NRGR, Phys.Rev. D73 (2006) 104031, [gr-qc/0511061].

[62] R. A. Porto and I. Z. Rothstein, The Hyperfine Einstein-Infeld-Hoffmann potential, Phys.Rev.Lett. 97 (2006) 021101, [gr-qc/0604099].

[63] R. A. Porto and I. Z. Rothstein, Spin(1)Spin(2) Effects in the Motion of Inspiralling Compact Binaries at Third Order in the Post-Newtonian Expansion, Phys.Rev. D78 (2008) 044012, [arXiv: 0802. 0720]. [Erratum-ibid. D81 (2010) 029904].

[64] M. Levi, Next to Leading Order gravitational Spin1-Spin2 coupling with Kaluza-Klein reduction, Phys.Rev. D82 (2010) 064029, [arXiv:0802.1508].

[65] R. A. Porto and I. Z. Rothstein, Next to Leading Order Spin(1)Spin(1) Effects in the Motion of Inspiralling Compact Binaries, Phys.Rev. D78 (2008) 044013, [arXiv:0804.0260]. [Erratum-ibid. D81 (2010) 029905].

[66] D. L. Perrodin, Subleading Spin-Orbit Correction to the Newtonian Potential in Effective Field Theory Formalism, in On recent developments in theoretical and experimental general 
relativity, astrophysics and relativistic field theories. Proceedings, 12th Marcel Grossmann Meeting on General Relativity, Paris, France, July 12-18, 2009. Vol. 1-3, pp. 725-727, 2010. arXiv: 1005.0634.

[67] R. A. Porto, Next to leading order spin-orbit effects in the motion of inspiralling compact binaries, Class.Quant.Grav. 27 (2010) 205001, [arXiv:1005.5730].

[68] M. Levi, Next to Leading Order gravitational Spin-Orbit coupling in an Effective Field Theory approach, Phys.Rev. D82 (2010) 104004, [arXiv:1006.4139].

[69] M. Levi, Binary dynamics from spin1-spin2 coupling at fourth post-Newtonian order, Phys.Rev. D85 (2012) 064043, [arXiv:1107.4322].

[70] M. Levi and J. Steinhoff, Equivalence of ADM Hamiltonian and Effective Field Theory approaches at next-to-next-to-leading order spin1-spin2 coupling of binary inspirals, JCAP 1412 (2014) 003, [arXiv: 1408.5762].

[71] M. Levi and J. Steinhoff, Next-to-next-to-leading order gravitational spin-squared potential via the effective field theory for spinning objects in the post-Newtonian scheme, JCAP 1601 (2016) 008, [arXiv:1506.05794].

[72] M. Levi and J. Steinhoff, Complete conservative dynamics for inspiralling compact binaries with spins at fourth post-Newtonian order, arXiv:1607.04252.

[73] M. Levi and J. Steinhoff, Leading order finite size effects with spins for inspiralling compact binaries, JHEP 06 (2015) 059, [arXiv:1410.2601].

[74] M. Levi and J. Steinhoff, Next-to-next-to-leading order gravitational spin-orbit coupling via the effective field theory for spinning objects in the post-Newtonian scheme, JCAP 1601 (2016) 011, [arXiv: 1506.05056].

[75] M. Levi and J. Steinhoff, Spinning gravitating objects in the effective field theory in the post-Newtonian scheme, JHEP 09 (2015) 219, [arXiv:1501.04956].

[76] M. Levi, Effective Field Theory of Post-Newtonian Gravity Including Spins, in 52nd Rencontres de Moriond on Gravitation (Moriond Gravitation 2017): La Thuile, Italy, March 25-April 1, 2017, pp. 263-267, 2017. arXiv:1705.07515.

[77] L. V. Delacrétaz, S. Endlich, A. Monin, R. Penco, and F. Riva, (Re-)Inventing the Relativistic Wheel: Gravity, Cosets, and Spinning Objects, JHEP 11 (2014) 008, [arXiv:1405.7384].

[78] W. D. Goldberger and I. Z. Rothstein, Dissipative effects in the worldline approach to black hole dynamics, Phys. Rev. D73 (2006) 104030, [hep-th/0511133].

[79] R. A. Porto, Absorption effects due to spin in the worldline approach to black hole dynamics, Phys. Rev. D77 (2008) 064026, [arXiv:0710.5150].

[80] B. Kol, The Delocalized Effective Degrees of Freedom of a Black Hole at Low Frequencies, Gen. Rel. Grav. 40 (2008) 2061-2068, [arXiv:0804.0187]. [Int. J. Mod. Phys.D17,2617(2009)].

[81] S. Endlich and R. Penco, Effective field theory approach to tidal dynamics of spinning astrophysical systems, Phys. Rev. D93 (2016) 064021, [arXiv:1510.08889].

[82] S. Endlich and R. Penco, A Modern Approach to Superradiance, JHEP 05 (2017) 052, [arXiv: 1609.06723].

[83] S. Chakrabarti, T. Delsate, and J. Steinhoff, New perspectives on neutron star and black 
hole spectroscopy and dynamic tides, arXiv:1304.2228.

[84] S. Chakrabarti, T. Delsate, and J. Steinhoff, Effective action and linear response of compact objects in Newtonian gravity, Phys. Rev. D88 (2013) 084038, [arXiv:1306.5820].

[85] J. Steinhoff, T. Hinderer, A. Buonanno, and A. Taracchini, Dynamical Tides in General Relativity: Effective Action and Effective-One-Body Hamiltonian, Phys. Rev. D94 (2016) 104028, [arXiv: 1608.01907].

[86] W. D. Goldberger and A. Ross, Gravitational radiative corrections from effective field theory, Phys.Rev. D81 (2010) 124015, [arXiv:0912.4254].

[87] A. Ross, Multipole expansion at the level of the action, Phys.Rev. D85 (2012) 125033, [arXiv: 1202 .4750].

[88] O. Birnholtz, S. Hadar, and B. Kol, Theory of post-Newtonian radiation and reaction, Phys.Rev. D88 (2013) 104037, [arXiv: 1305.6930].

[89] O. Birnholtz, S. Hadar, and B. Kol, Radiation reaction at the level of the action, Int. J. Mod. Phys. A29 (2014) 1450132, [arXiv:1402.2610].

[90] R. A. Porto, A. Ross, and I. Z. Rothstein, Spin induced multipole moments for the gravitational wave flux from binary inspirals to third Post-Newtonian order, JCAP 1103 (2011) 009, [arXiv: 1007.1312].

[91] R. A. Porto, A. Ross, and I. Z. Rothstein, Spin induced multipole moments for the gravitational wave amplitude from binary inspirals to 2.5 Post-Newtonian order, JCAP 1209 (2012) 028, [arXiv:1203.2962].

[92] W. D. Goldberger, A. Ross, and I. Z. Rothstein, Black hole mass dynamics and renormalization group evolution, Phys. Rev. D89 (2014) 124033, [arXiv:1211.6095].

[93] L. Barack and A. Pound, Self-force and radiation reaction in general relativity, Rept. Prog. Phys. 82 (2019) 016904, [arXiv: 1805.10385].

[94] C. R. Galley and B. Hu, Self-force on extreme mass ratio inspirals via curved spacetime effective field theory, Phys.Rev. D79 (2009) 064002, [arXiv:0801.0900].

[95] C. R. Galley, A nonlinear scalar model of extreme mass ratio inspirals in effective field theory I. Self force through third order, Class.Quant.Grav. 29 (2012) 015010, [arXiv: 1012.4488].

[96] C. R. Galley, A Nonlinear scalar model of extreme mass ratio inspirals in effective field theory II. Scalar perturbations and a master source, Class.Quant.Grav. 29 (2012) 015011, [arXiv:1107.0766].

[97] C. R. Galley and R. A. Porto, Gravitational self-force in the ultra-relativistic limit: the "large- $N "$ expansion, JHEP 1311 (2013) 096, [arXiv: 1302.4486].

[98] P. Zimmerman, Gravitational self-force in nonvacuum spacetimes: An effective field theory derivation, Phys. Rev. D92 (2015) 064040, [arXiv:1505.03915].

[99] C. R. Galley and M. Tiglio, Radiation reaction and gravitational waves in the effective field theory approach, Phys.Rev. D79 (2009) 124027, [arXiv:0903.1122].

[100] C. R. Galley and A. K. Leibovich, Radiation reaction at 3.5 post-Newtonian order in effective field theory, Phys.Rev. D86 (2012) 044029, [arXiv:1205.3842].

[101] S. Foffa and R. Sturani, Tail terms in gravitational radiation reaction via effective field 
theory, Phys.Rev. D87 (2013) 044056, [arXiv:1111.5488].

[102] C. R. Galley, A. K. Leibovich, R. A. Porto, and A. Ross, Tail effect in gravitational radiation reaction: Time nonlocality and renormalization group evolution, Phys. Rev. D93 (2016) 124010, [arXiv:1511.07379].

[103] C. R. Galley, Classical Mechanics of Nonconservative Systems, Phys.Rev.Lett. 110 (2013) 174301, [arXiv:1210.2745].

[104] C. R. Galley, D. Tsang, and L. C. Stein, The principle of stationary nonconservative action for classical mechanics and field theories, arXiv:1412.3082.

[105] O. Birnholtz and S. Hadar, Action for reaction in general dimension, Phys. Rev. D89 (2014) 045003, [arXiv:1311.3196].

[106] O. Birnholtz and S. Hadar, Gravitational radiation-reaction in arbitrary dimension, Phys. Rev. D91 (2015) 124065, [arXiv:1501.06524].

[107] N. T. Maia, C. R. Galley, A. K. Leibovich, and R. A. Porto, Radiation reaction for spinning bodies in effective field theory I: Spin-orbit effects, Phys. Rev. D96 (2017) 084064, [arXiv: 1705.07934].

[108] N. T. Maia, C. R. Galley, A. K. Leibovich, and R. A. Porto, Radiation reaction for spinning bodies in effective field theory II: Spin-spin effects, Phys. Rev. D96 (2017) 084065, [arXiv: 1705.07938].

[109] A. D. Fokker, Ein invarianter Variationssatz für die Bewegung mehrerer elektrischer Massenteilchen, Z. Physik 58 (1929) 386-393.

[110] T. Damour and G. Esposito-Farese, Testing gravity to second postNewtonian order: A Field theory approach, Phys.Rev. D53 (1996) 5541-5578, [gr-qc/9506063].

[111] T. Damour and G. Esposito-Farese, Gravitational wave versus binary - pulsar tests of strong field gravity, Phys.Rev. D58 (1998) 042001, [gr-qc/9803031].

[112] M. Levi and J. Steinhoff, EFTofPNG: A package for high precision computation with the Effective Field Theory of Post-Newtonian Gravity, Class. Quant. Grav. 34 (2017) 244001, [arXiv: 1705.06309].

[113] "Black Hole Perturbation Toolkit." http://bhptoolkit.org/.

[114] S. Foffa and R. Sturani, Effective field theory methods to model compact binaries, Classical and Quantum Gravity 31 (2014) 043001, [arXiv:1309.3474].

[115] I. Z. Rothstein, Progress in effective field theory approach to the binary inspiral problem, Gen.Rel.Grav. 46 (2014) 1726.

[116] R. A. Porto, The effective field theorist's approach to gravitational dynamics, Phys. Rept. 633 (2016) 1-104, [arXiv: 1601.04914].

[117] M. E. Peskin and D. V. Schroeder, An Introduction to quantum field theory. Addison-Wesley, Reading, USA, 1995.

[118] D. Binosi and L. Theussl, JaxoDraw: A Graphical user interface for drawing Feynman diagrams, Comput. Phys. Commun. 161 (2004) 76-86, [hep-ph/0309015].

[119] D. Binosi, J. Collins, C. Kaufhold, and L. Theussl, JaxoDraw: A Graphical user interface for drawing Feynman diagrams. Version 2.0 release notes, Comput. Phys. Commun. 180 (2009) 1709-1715, [arXiv:0811.4113]. 
[120] J. A. M. Vermaseren, Axodraw, Comput. Phys. Commun. 83 (1994) 45-58.

[121] T. Appelquist and J. Carazzone, Infrared Singularities and Massive Fields, Phys. Rev. D11 (1975) 2856.

[122] A. J. Hanson and T. Regge, The Relativistic Spherical Top, Annals Phys. 87 (1974) 498.

[123] I. Bailey and W. Israel, Lagrangian Dynamics of Spinning Particles and Polarized Media in General Relativity, Commun.Math.Phys. 42 (1975) 65-82.

[124] K. Yee and M. Bander, Equations of motion for spinning particles in external electromagnetic and gravitational fields, Phys.Rev. D48 (1993) 2797-2799, [hep-th/9302117].

[125] W. Tulczyjew, Motion of multipole particles in general relativity theory, Acta Phys.Polon. 18 (1959) 393.

[126] H. Ruegg and M. Ruiz-Altaba, The Stueckelberg field, Int. J. Mod. Phys. A19 (2004) 3265-3348, [hep-th/0304245].

[127] J. D. Jackson, Classical Electrodynamics. Wiley, 1998.

[128] V. Cardoso, O. J. Dias, and P. Figueras, Gravitational radiation in $d>4$ from effective field theory, Phys.Rev. D78 (2008) 105010, [arXiv:0807.2261].

[129] T. Damour and P. Jaranowski, Four-loop static contribution to the gravitational interaction potential of two point masses, Phys. Rev. D95 (2017) 084005, [arXiv:1701.02645].

[130] B. Kol and M. Smolkin, Dressing the Post-Newtonian two-body problem and Classical Effective Field Theory, Phys.Rev. D80 (2009) 124044, [arXiv:0910.5222].

[131] B. Kol and R. Shir, Classical 3-loop 2-body diagrams, JHEP 09 (2013) 069, [arXiv:1306.3220].

[132] R. A. Porto and I. Z. Rothstein, Apparent ambiguities in the post-Newtonian expansion for binary systems, Phys. Rev. D96 (2017) 024062, [arXiv:1703.06433].

[133] T. Damour, M. Soffel, and C.-m. Xu, General relativistic celestial mechanics. 1. Method and definition of reference systems, Phys.Rev. D43 (1991) 3272-3307.

[134] J. S. Schwinger, Quantized gravitational field, Phys.Rev. 130 (1963) 1253-1258.

[135] M. Pryce, The Mass center in the restricted theory of relativity and its connection with the quantum theory of elementary particles, Proc.Roy.Soc.Lond. A195 (1948) 62-81.

[136] T. Newton and E. P. Wigner, Localized States for Elementary Systems, Rev.Mod.Phys. 21 (1949) 400-406.

[137] V. A. Smirnov, Feynman integral calculus. Springer, Berlin, Germany, 2006.

[138] P. Jaranowski and G. Schäfer, Towards the 4th post-Newtonian Hamiltonian for two-point-mass systems, Phys.Rev. D86 (2012) 061503, [arXiv:1207.5448].

[139] P. Jaranowski and G. Schäfer, Dimensional regularization of local singularities in the 4 th post-Newtonian two-point-mass Hamiltonian, Phys.Rev. D87 (2013) 081503, [arXiv: 1303.3225].

[140] T. Damour, P. Jaranowski, and G. Schäfer, Nonlocal-in-time action for the fourth post-Newtonian conservative dynamics of two-body systems, Phys.Rev. D89 (2014) 064058, [arXiv:1401.4548]. 
[141] T. Damour, P. Jaranowski, and G. Schäfer, Conservative dynamics of two-body systems at the fourth post-Newtonian approximation of general relativity, Phys. Rev. D93 (2016) 084014, [arXiv: 1601.01283].

[142] L. Bernard, L. Blanchet, A. Bohé, G. Faye, and S. Marsat, Fokker action of nonspinning compact binaries at the fourth post-Newtonian approximation, Phys. Rev. D93 (2016) 084037, [arXiv: 1512.02876].

[143] L. Bernard, L. Blanchet, A. Bohé, G. Faye, and S. Marsat, Energy and periastron advance of compact binaries on circular orbits at the fourth post-Newtonian order, Phys. Rev. D95 (2017) 044026, [arXiv: 1610.07934].

[144] L. Bernard, L. Blanchet, A. Bohé, G. Faye, and S. Marsat, Dimensional regularization of the IR divergences in the Fokker action of point-particle binaries at the fourth post-Newtonian order, Phys. Rev. D96 (2017) 104043, [arXiv:1706.08480].

[145] B. Barker and R. O'Connell, Acceleration-dependent lagrangians and equations of motion, Phys.Lett. A78 (1980) 231-232.

[146] G. Schäfer, Acceleration-dependent lagrangians in general relativity, Phys.Lett. A100 (1984) 128-129.

[147] T. Damour and G. Schäfer, Lagrangians for $n$ point masses at the second post-Newtonian approximation of general relativity, Gen.Rel.Grav. 17 (1985) 879-905.

[148] T. Damour and G. Schäfer, Redefinition of position variables and the reduction of higher order Lagrangians, J.Math.Phys. 32 (1991) 127-134.

[149] H. Goldstein, Classical Mechanics. Addison-Wesley, Second ed., 1980.

[150] J. S. Schwinger, Brownian motion of a quantum oscillator, J. Math. Phys. 2 (1961) 407-432.

[151] L. V. Keldysh, Diagram technique for nonequilibrium processes, Zh. Eksp. Teor. Fiz. 47 (1964) 1515-1527. [Sov. Phys. JETP20,1018(1965)].

[152] E. A. Calzetta and B.-L. B. Hu, Nonequilibrium Quantum Field Theory. Cambridge University Press, 2008.

[153] C. R. Galley, A. K. Leibovich, and I. Z. Rothstein, Finite size corrections to the radiation reaction force in classical electrodynamics, Phys.Rev.Lett. 105 (2010) 094802, [arXiv:1005.2617].

[154] P. Forgacs, T. Herpay, and P. Kovacs, Comment on 'Finite Size Corrections to the Radiation Reaction Force in Classical Electrodynamics', Phys.Rev.Lett. 109 (2012) 029501, [arXiv: 1202.6289].

[155] C. R. Galley, A. K. Leibovich, and I. Z. Rothstein, Reply to 'Comment on 'Finite size corrections to the radiation reaction force in classical electrodynamics", Phys.Rev.Lett. 109 (2012) 029502, [arXiv: 1206.4773].

[156] R. A. Porto, Lamb shift and the gravitational binding energy for binary black holes, Phys. Rev. D96 (2017) 024063, [arXiv:1703.06434].

[157] D. Gerosa and M. Kesden, PRECESSION: Dynamics of spinning black-hole binaries with python, Phys. Rev. D93 (2016) 124066, [arXiv:1605.01067].

[158] T. Damour, A. Nagar, D. Pollney, and C. Reisswig, Energy versus Angular Momentum in Black Hole Binaries, Phys.Rev.Lett. 108 (2012) 131101, [arXiv:1110.2938]. 
[159] A. Le Tiec, A. H. Mroue, L. Barack, A. Buonanno, H. P. Pfeiffer, N. Sago, and A. Taracchini, Periastron Advance in Black Hole Binaries, Phys. Rev. Lett. 107 (2011) 141101, [arXiv:1106.3278].

[160] A. Le Tiec et al., Periastron Advance in Spinning Black Hole Binaries: Gravitational Self-Force from Numerical Relativity, Phys. Rev. D88 (2013) 124027, [arXiv:1309.0541].

[161] T. Hinderer et al., Periastron advance in spinning black hole binaries: comparing effective-one-body and Numerical Relativity, Phys. Rev. D88 (2013) 084005, [arXiv: 1309.0544].

[162] C. R. Galley and I. Z. Rothstein, Deriving analytic solutions for compact binary inspirals without recourse to adiabatic approximations, Phys. Rev. D95 (2017) 104054, [arXiv: 1609.08268].

[163] R. Britto, F. Cachazo, and B. Feng, New recursion relations for tree amplitudes of gluons, Nucl. Phys. B715 (2005) 499-522, [hep-th/0412308].

[164] D. Neill and I. Z. Rothstein, Classical Space-Times from the S Matrix, Nucl. Phys. B877 (2013) 177-189, [arXiv: 1304.7263].

[165] V. Vaidya, Gravitational spin Hamiltonians from the S matrix, Phys. Rev. D91 (2015) 024017, [arXiv: 1410.5348].

[166] N. E. J. Bjerrum-Bohr, J. F. Donoghue, and P. Vanhove, On-shell Techniques and Universal Results in Quantum Gravity, JHEP 02 (2014) 111, [arXiv:1309.0804].

[167] N. E. J. Bjerrum-Bohr, J. F. Donoghue, B. R. Holstein, L. Planté, and P. Vanhove, Bending of Light in Quantum Gravity, Phys. Rev. Lett. 114 (2015) 061301, [arXiv:1410.7590].

[168] N. E. J. Bjerrum-Bohr, J. F. Donoghue, B. R. Holstein, L. Planté, and P. Vanhove, Light-like Scattering in Quantum Gravity, JHEP 11 (2016) 117, [arXiv:1609.07477].

[169] F. Cachazo and A. Guevara, Leading Singularities and Classical Gravitational Scattering, JHEP 02 (2020) 181, [arXiv: 1705.10262].

[170] A. Guevara, Holomorphic Classical Limit for Spin Effects in Gravitational and Electromagnetic Scattering, JHEP 04 (2019) 033, [arXiv: 1706. 02314].

[171] T. Damour, F. Guercilena, I. Hinder, S. Hopper, A. Nagar, and L. Rezzolla, Strong-Field Scattering of Two Black Holes: Numerics Versus Analytics, Phys. Rev. D89 (2014) 081503, [arXiv:1402.7307].

[172] T. Damour, Gravitational scattering, post-Minkowskian approximation and Effective One-Body theory, Phys. Rev. D94 (2016) 104015, [arXiv:1609.00354].

[173] J. Vines, Scattering of two spinning black holes in post-Minkowskian gravity, to all orders in spin, and effective-one-body mappings, Class. Quant. Grav. 35 (2018) 084002, [arXiv:1709.06016].

[174] T. Damour, High-energy gravitational scattering and the general relativistic two-body problem, Phys. Rev. D97 (2018) 044038, [arXiv:1710.10599].

[175] N. E. J. Bjerrum-Bohr, P. H. Damgaard, G. Festuccia, L. Planté, and P. Vanhove, General Relativity from Scattering Amplitudes, Phys. Rev. Lett. 121 (2018) 171601, [arXiv: 1806.04920].

[176] C. Cheung, I. Z. Rothstein, and M. P. Solon, From Scattering Amplitudes to Classical Potentials in the Post-Minkowskian Expansion, Phys. Rev. Lett. 121 (2018) 251101, 
[arXiv: 1808.02489].

[177] H. Kawai, D. C. Lewellen, and S. H. H. Tye, A Relation Between Tree Amplitudes of Closed and Open Strings, Nucl. Phys. B269 (1986) 1-23.

[178] Z. Bern, J. J. M. Carrasco, and H. Johansson, New Relations for Gauge-Theory Amplitudes, Phys. Rev. D78 (2008) 085011, [arXiv:0805.3993].

[179] Z. Bern, J. J. M. Carrasco, and H. Johansson, Perturbative Quantum Gravity as a Double Copy of Gauge Theory, Phys. Rev. Lett. 105 (2010) 061602, [arXiv:1004.0476].

[180] Z. Bern, T. Dennen, Y.-t. Huang, and M. Kiermaier, Gravity as the Square of Gauge Theory, Phys. Rev. D82 (2010) 065003, [arXiv:1004.0693].

[181] J. J. M. Carrasco, Gauge and Gravity Amplitude Relations, in Proceedings, Theoretical Advanced Study Institute in Elementary Particle Physics: Journeys Through the Precision Frontier: Amplitudes for Colliders (TASI 2014): Boulder, Colorado, June 2-27, 2014, pp. 477-557, WSP, 2015. arXiv:1506.00974.

[182] A. Luna, R. Monteiro, I. Nicholson, D. O'Connell, and C. D. White, The double copy: Bremsstrahlung and accelerating black holes, JHEP 06 (2016) 023, [arXiv:1603.05737].

[183] W. D. Goldberger and A. K. Ridgway, Radiation and the classical double copy for color charges, Phys. Rev. D95 (2017) 125010, [arXiv:1611.03493].

[184] A. Luna, R. Monteiro, I. Nicholson, A. Ochirov, D. O'Connell, N. Westerberg, and C. D. White, Perturbative spacetimes from Yang-Mills theory, JHEP 04 (2017) 069, [arXiv: 1611.07508].

[185] C. Cheung, C.-H. Shen, and C. Wen, Unifying Relations for Scattering Amplitudes, JHEP 02 (2018) 095, [arXiv:1705.03025].

[186] W. D. Goldberger, S. G. Prabhu, and J. O. Thompson, Classical gluon and graviton radiation from the bi-adjoint scalar double copy, Phys. Rev. D96 (2017) 065009, [arXiv: 1705.09263].

[187] T. Adamo, E. Casali, L. Mason, and S. Nekovar, Scattering on plane waves and the double copy, Class. Quant. Grav. 35 (2018) 015004, [arXiv: 1706.08925].

[188] A. Luna, I. Nicholson, D. O'Connell, and C. D. White, Inelastic Black Hole Scattering from Charged Scalar Amplitudes, JHEP 03 (2018) 044, [arXiv:1711.03901].

[189] W. D. Goldberger and A. K. Ridgway, Bound states and the classical double copy, Phys. Rev. D97 (2018) 085019, [arXiv:1711.09493].

[190] D. Chester, Radiative double copy for Einstein-Yang-Mills theory, Phys. Rev. D97 (2018) 084025, [arXiv:1712.08684].

[191] W. D. Goldberger, J. Li, and S. G. Prabhu, Spinning particles, axion radiation, and the classical double copy, Phys. Rev. D97 (2018) 105018, [arXiv:1712.09250].

[192] J. Li and S. G. Prabhu, Gravitational radiation from the classical spinning double copy, Phys. Rev. D97 (2018) 105019, [arXiv:1803.02405].

[193] A. Ilderton, Screw-symmetric gravitational waves: a double copy of the vortex, Phys. Lett. B782 (2018) 22-27, [arXiv: 1804.07290].

[194] C.-H. Shen, Gravitational Radiation from Color-Kinematics Duality, JHEP 11 (2018) 162, [arXiv: 1806.07388]. 
[195] A. Strominger, Lectures on the Infrared Structure of Gravity and Gauge Theory, arXiv: 1703.05448.

[196] A. Strominger and A. Zhiboedov, Gravitational Memory, BMS Supertranslations and Soft Theorems, JHEP 01 (2016) 086, [arXiv:1411.5745].

[197] S. Pasterski, A. Strominger, and A. Zhiboedov, New Gravitational Memories, JHEP 12 (2016) 053, [arXiv:1502.06120].

[198] E. E. Flanagan and D. A. Nichols, Observer dependence of angular momentum in general relativity and its relationship to the gravitational-wave memory effect, Phys. Rev. D92 (2015) 084057, [arXiv: 1411.4599]. [Erratum: Phys. Rev.D93,049905(2016)].

[199] D. A. Nichols, Spin memory effect for compact binaries in the post-Newtonian approximation, Phys. Rev. D95 (2017) 084048, [arXiv:1702.03300].

[200] Y.-Z. Chu, Transverse traceless gravitational waves in a spatially flat FLRW universe: Causal structure from dimensional reduction, Phys. Rev. D92 (2015) 124038, [arXiv: 1504.06337].

[201] Y.-Z. Chu, More On Cosmological Gravitational Waves And Their Memories, Class. Quant. Grav. 34 (2017) 194001, [arXiv: 1611.00018].

[202] A. Laddha and A. Sen, Gravity Waves from Soft Theorem in General Dimensions, JHEP 09 (2018) 105, [arXiv: 1801.07719].

[203] A. Laddha and A. Sen, Observational Signature of the Logarithmic Terms in the Soft Graviton Theorem, Phys. Rev. D100 (2019) 024009, [arXiv:1806.01872].

[204] K. Hinterbichler, Theoretical Aspects of Massive Gravity, Rev. Mod. Phys. 84 (2012) 671-710, [arXiv:1105.3735].

[205] C. de Rham, Massive Gravity, Living Rev. Rel. 17 (2014) 7, [arXiv:1401.4173].

[206] C. Deffayet and D. A. Steer, A formal introduction to Horndeski and Galileon theories and their generalizations, Class. Quant. Grav. 30 (2013) 214006, [arXiv:1307.2450].

[207] T. Clifton, P. G. Ferreira, A. Padilla, and C. Skordis, Modified Gravity and Cosmology, Phys. Rept. 513 (2012) 1-189, [arXiv:1106. 2476].

[208] A. Joyce, B. Jain, J. Khoury, and M. Trodden, Beyond the Cosmological Standard Model, Phys. Rept. 568 (2015) 1-98, [arXiv:1407.0059].

[209] U. Cannella, S. Foffa, M. Maggiore, H. Sanctuary, and R. Sturani, Extracting the three and four-graviton vertices from binary pulsars and coalescing binaries, Phys.Rev. D80 (2009) 124035, [arXiv:0907.2186].

[210] C. de Rham, J. T. Deskins, A. J. Tolley, and S.-Y. Zhou, Graviton Mass Bounds, Rev. Mod. Phys. 89 (2017) 025004, [arXiv: 1606.08462].

[211] V. Cardoso, G. Castro, and A. Maselli, Gravitational waves in massive gravity theories: waveforms, fluxes and constraints from extreme-mass-ratio mergers, Phys. Rev. Lett. 121 (2018) 251103, [arXiv: 1809.00673].

[212] U. Cannella and R. Sturani, Classical energy momentum tensor renormalisation via effective field theory methods, Gen. Rel. Grav. 42 (2010) 2491-2509, [arXiv:0808.4034].

[213] H. Sanctuary and R. Sturani, Effective field theory analysis of the self-interacting chameleon, Gen. Rel. Grav. 42 (2010) 1953-1967, [arXiv:0809.3156]. 
[214] Y.-Z. Chu and M. Trodden, Retarded Green's function of a Vainshtein system and Galileon waves, Phys. Rev. D87 (2013) 024011, [arXiv:1210.6651].

[215] C. de Rham, A. J. Tolley, and D. H. Wesley, Vainshtein Mechanism in Binary Pulsars, Phys. Rev. D87 (2013) 044025, [arXiv: 1208.0580].

[216] C. de Rham, A. Matas, and A. J. Tolley, Galileon Radiation from Binary Systems, Phys. Rev. D87 (2013) 064024, [arXiv: 1212.5212].

[217] F. Dar, C. De Rham, J. T. Deskins, J. T. Giblin, and A. J. Tolley, Scalar Gravitational Radiation from Binaries: Vainshtein Mechanism in Time-dependent Systems, Class. Quant. Grav. 36 (2019) 025008, [arXiv: 1808.02165]. 